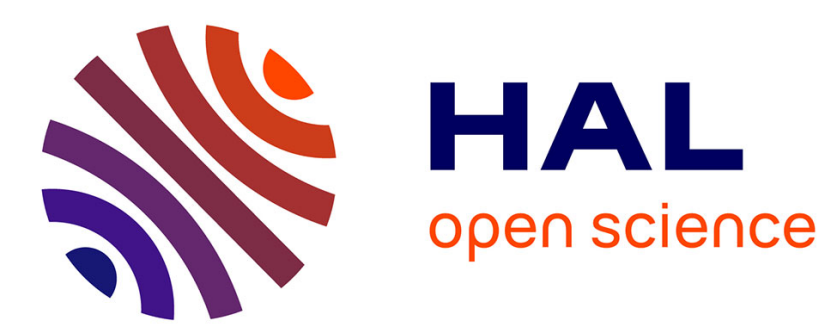

\title{
Stability of a class of nonlinear reaction-diffusion equations and stochastic homogenization
}

\author{
Omar Anza Hafsa, Jean Philippe Mandallena, Gérard Michaille
}

\section{To cite this version:}

Omar Anza Hafsa, Jean Philippe Mandallena, Gérard Michaille. Stability of a class of nonlinear reaction-diffusion equations and stochastic homogenization. Asymptotic Analysis, 2019, 115 (3-4), pp.169-221. 10.3233/ASY-191531 . hal-01928187

\section{HAL Id: hal-01928187 \\ https://hal.science/hal-01928187}

Submitted on 20 Nov 2018

HAL is a multi-disciplinary open access archive for the deposit and dissemination of scientific research documents, whether they are published or not. The documents may come from teaching and research institutions in France or abroad, or from public or private research centers.
L'archive ouverte pluridisciplinaire HAL, est destinée au dépôt et à la diffusion de documents scientifiques de niveau recherche, publiés ou non, émanant des établissements d'enseignement et de recherche français ou étrangers, des laboratoires publics ou privés. 


\title{
STABILITY OF A CLASS OF NONLINEAR REACTION-DIFFUSION EQUATIONS AND STOCHASTIC HOMOGENIZATION
}

\author{
OMAR ANZA HAFSA, JEAN PHILIPPE MANDALLENA, AND GÉRARD MICHAILLE
}

\begin{abstract}
We establish a convergence theorem for a class of nonlinear reaction-diffusion equations when the diffusion term is the subdifferential of a convex functional in a class of functionals of the calculus of variations equipped with the Mosco-convergence. The reaction term, which is not globally Lipschitz with respect to the state variable, gives rise to bounded solutions, and cover a wide variety of models. As a consequence we prove a homogenization theorem for this class under a stochastic homogenization framework.
\end{abstract}

\section{INTRODUCTION}

Let $\Omega$ be a bounded regular domain in $\mathbb{R}^{N}$, and $T$ any positive real number. The purpose of this paper is to investigate the stability and the stochastic homogenization of the class of reaction-diffusion problems

$$
(\mathcal{P})\left\{\begin{array}{l}
\frac{d u}{d t}(t, \cdot)+\partial \Phi(u(t, \cdot)) \ni F(t, u(t, \cdot)) \text { for a.e. } t \in(0, T) \\
u(0, \cdot)=u^{0}, u^{0} \in \overline{\operatorname{dom}(\partial \Phi)}, u^{0} \text { suitably bounded according to } F,
\end{array}\right.
$$

defined in $L^{2}\left(0, T, L^{2}(\Omega)\right)$, when $\Phi$ belongs to the class of integral functionals of the type

$$
\Phi(u)=\left\{\begin{array}{cl}
\int_{\Omega} W(x, \nabla u(x)) d x+\frac{1}{2} \int_{\partial \Omega} a_{0} u^{2} d \mathcal{H}_{N-1}-\int_{\partial \Omega} h u d \mathcal{H}_{N-1} & \text { if } u \in H^{1}(\Omega) \\
+\infty & \text { otherwise. }
\end{array}\right.
$$

The reaction functionals $F$, called CP-structured reaction functionals, have the following form:

$$
\forall v \in L^{2}(\Omega), F(t, v)(x)=f(t, x, v(x)), f(t, x, \zeta)=r(t, x) \cdot g(\zeta)+q(t, x),
$$

where $g: \mathbb{R} \rightarrow \mathbb{R}^{l}$ is a locally Lipschitz function, $r \in W^{1,1}\left(0, T, L_{\text {loc }}^{2}\left(\mathbb{R}^{N}, \mathbb{R}^{l}\right)\right) \cap L^{\infty}\left([0, T] \times \mathbb{R}^{N}, \mathbb{R}^{l}\right)$, and $q \in W^{1,1}\left(0, T, L_{\text {loc }}^{2}\left(\mathbb{R}^{N}\right)\right) \cap L^{2}\left(0, T, L_{\text {loc }}^{2}\left(\mathbb{R}^{N}\right)\right)$. We assume furthermore that $f$ satisfies a condition $(\mathrm{CP})$ $((\mathrm{CP})$ for Comparison Principle, see Definition 3.1). Under this condition, $(\mathcal{P})$ admits a unique bounded solution according to $u_{0}$, with a right derivative at every $t \in(0, T)$ (Theorems 3.1. 3.2. Problems $(\mathcal{P})$ cover a wide variety of applications in the fields of thermochemistry, combustion, biochemical systems, as well as those of population dynamics and evolution of ecosystems as illustrated in examples A.1, A.2. A.3. A.4 of the Appendix A.

The main first result of the paper, Theorem 4.1, states the stability of the class $(\mathcal{P})$ when the class of functionals $\Phi$ is equipped with the $\Gamma$-convergence associated both with strong and weak topology of $L^{2}(\Omega)$, namely, the Mosco-convergence, and the class of functionals $F$ with some "weak" convergence. Stochastic homogenization of reaction-diffusion problems $(\mathcal{P})$ is addressed in Section 5 w here we set up the basic concepts concerning ergodic dynamical systems. The main theorem, Theorem 5.1, based on Theorem 4.1, states the limit homogenized problem of $\left(\mathcal{P}_{\varepsilon}(\omega)\right)$ when $\varepsilon \rightarrow 0$. As an example, in the appendix B, the stochastic homogenization of the reaction-diffusion problem describing a food-limited population model is treated in two differents situations. The reaction functional is that of the Fisher

(Omar Anza Hafsa, Jean Philippe Mandallena, Gérard Michaille) UNIVERSITE DE NIMES, Laboratoire MIPA, Site des Carmes, Place Gabriel Péri, 30021 Nîmes, France.

E-mail addresses: (Omar Anza Hafsa, Jean Philippe Mandallena, Gérard Michaille) omar.anza-hafsa@unimes.fr, jean-philippe.mandallena@unimes.fr, gerard.michaille@gmail.com.

1991 Mathematics Subject Classification. 35K57, 35B27, 49J45.

Key words and phrases. Convergence of reaction-diffusion equations, stochastic homogenization, separate variables reaction functionals. 
model with Allee effect (see Example A.1 a)). In the first situation the small spatial heterogeneities of size $\varepsilon$ are distributed following a random patch model, i.e., a random checkerboard-like environment. In the second situation, the discrete dynamical system describes spatial heterogeneities distributed following a Poisson point process. For homogenization of convection-diffusion equations, and parabolic problems in perforated domains or in periodic or random environments, we refer the reader to [2, 3, 4, 5, 15, and references therein. For homogenization of a Fokker-Planck equation with space-time periodic potential, we refer to 22 .

A similar analysis will be performed from this paper for time delays reaction-diffusion equations and coupled reaction-diffusion systems in forthcoming works. For these problems, the reaction functionals are of the form $F(t, u, v)(x)=f(t, x, u(x), v(x))$ for all $u$ and $v$ in $L^{2}(\Omega)$, where $f\left(t, x, \zeta, \zeta^{\prime}\right)=$ $r(t, x) \odot g_{1}\left(\zeta^{\prime}\right) \cdot g_{2}(\zeta)+q(t, x)$ ] under the condition that for fixed $\zeta^{\prime} \in \mathbb{R}, \zeta \mapsto f\left(t, \zeta, \zeta^{\prime}\right)$ is a CPstructured reaction function.

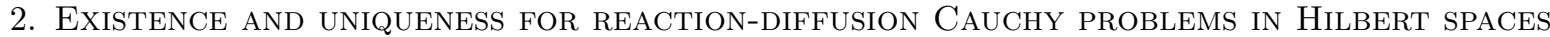

In this section, $X$ denotes a Hilbert space equipped with its scalar product denoted by $\langle\cdot, \cdot\rangle$ and its associated norm $\|\cdot\|_{X}$. In all along the paper we use the same notation $|\cdot|$ to denote the norms of the euclidean spaces $\mathbb{R}^{d}, d \geq 1$, and by $\xi \cdot \xi^{\prime}$ the standard scalar product of two elements $\xi, \xi^{\prime}$ in $\mathbb{R}^{d}$.

Let $\Phi: X \rightarrow \mathbb{R} \cup\{+\infty\}$ be a convex proper lower semicontinuous (lsc in short) functional, minorized, i.e., satisfying $\inf _{X} \Phi>-\infty$, that we assume to be Gâteaux differentiable so that its subdifferential $\partial \Phi$ is single valued. We make this choice in order to simplify the notation but, in this section, we could use the subdifferential of $\Phi$ in place of its Gâteaux derivative, denoted by $D \Phi$, without additional difficulties. We denote by $\operatorname{dom}(\Phi)$ and $\operatorname{dom}(D \Phi)$ the domain of $\Phi$ and $D \Phi$ respectively.

On the other hand, let $F:[0,+\infty) \times X \rightarrow X$ be a Borel measurable map fulfilling the two following conditions:

$\left(\mathrm{C}_{1}\right)$ there exists $L \in L_{\text {loc }}^{2}(0,+\infty)$ such that $\|F(t, u)-F(t, v)\|_{X} \leq L(t)\|u-v\|_{X}$ for all $(u, v) \in X^{2}$ and all $t>0$

$\left(\mathrm{C}_{2}\right)$ the map $t \mapsto\|F(t, 0)\|_{X}$ belongs to $L_{\text {loc }}^{2}(0,+\infty)$.

Given $T>0$ and $u_{0}$ in $\overline{\operatorname{dom}(D \Phi)}$, the map $F$ is referred to as the reaction part, and $D \Phi$ as the diffusion part of the following Cauchy problem:

$$
(\mathcal{P})\left\{\begin{array}{l}
\frac{d u}{d t}(t)+D \Phi(u(t))=F(t, u(t)) \text { for a.e. } t \in(0, T) \\
u(0)=u_{0}, u_{0} \in \overline{\operatorname{dom}(D \Phi)} .
\end{array}\right.
$$

where $\frac{d u}{d t}$ denotes the distributional time derivative of $u$. We say that $u$ is a solution of $(\mathcal{P})$ if $u \in$ $L^{2}([0, T], X)$ is absolutely continuous in time and satisfies $(\mathcal{P})$. In all the paper, the space $C([0, T], X)$ is endowed with the sup-norm. The results stated in the theorem below are somewhat well known. The proof is based on [8, Theorems 17.2.5, 17.2.6], or on [13, Theorem 3.7]), together with a fixed point procedure.

Theorem 2.1 (local existence). Assume that $F$ satisfies conditions $\left(\mathrm{C}_{1}\right),\left(\mathrm{C}_{2}\right)$, Then, there exist $T>0$ small enough and a unique solution $u \in C([0, T], X)$ of $(\mathcal{P})$ which satisfies

$\left(\mathrm{L}_{1}\right) u(t) \in \operatorname{dom}(D \Phi)$ for a.e. $t \in(0, T)$,

$\left(\mathrm{L}_{2}\right) u$ is almost everywhere differentiable in $(0, T)$ and $u^{\prime}(t)=\frac{d u}{d t}(t)$ for a.e. $t \in(0, T)$.

Assume furthermore that $G:[0, T] \rightarrow X$ defined by $G(t)=F(t, u(t))$ belongs to $W^{1,1}(0, T, X)$, then u satisfies:

$\left(\mathrm{L}_{3}\right) u(t) \in \operatorname{dom}(D \Phi)$ for all $\left.\left.t \in\right] 0, T\right]$

$u$ admits a right derivative $\frac{d u^{+}}{d t}(t)$ at every $t \in(0, T)$ and $\frac{d u^{+}}{d t}(t)+D \Phi(u(t))=F(t, u(t))$.

Denote by $T^{*}>0$ a small enough real number so that $(\mathcal{P})$ admits a unique solution in $C\left(\left[0, T^{*}\right], X\right)$, whose existence is asserted in Theorem 2.1. Under the initial condition $u_{0} \in \overline{\operatorname{dom}(D \Phi)}$ we are not assured

\footnotetext{
${ }^{1}$ We denote by $\xi \odot \xi^{\prime}$ the Hadamard (or Schur) product of two elements $\xi$ and $\xi^{\prime}$ in $\mathbb{R}^{l}$.
} 
that the derivative $\frac{d u}{d t}$ of the solution belongs to $L^{2}\left(0, T^{*}, X\right)$. Nevertheless $\sqrt{t} \frac{d u}{d t} \in L^{2}\left(0, T^{*}, X\right)$ (see [8, Theorem 17.2.5] or [13, Theorem 3.6]). Hence, for $0<\delta<T^{*}, \frac{d u}{d t}$ belongs to $L^{2}\left(\delta, T^{*}, X\right)$. Set

$$
E:=\{T>\delta: \exists u \in C([0, T], X) \text { solution of }(\mathcal{P})\} .
$$

Since $T^{*} \in E$, we have $E \neq \emptyset$. We define the maximal time in $\overline{\mathbb{R}}_{+}$by $T_{M a x}:=\sup E$ and denote by $u$ the maximal solution of $(\mathcal{P})$ in $C\left(\left[0, T_{M a x}\right), X\right)$. We have the following alternative:

Theorem 2.2 (Global existence or blow-up in finite time). Assume that $F$ satisfies $\left(\mathrm{C}_{1}\right),\left(\mathrm{C}_{2}\right)$, then we have the blow-up alternative

$\left(\mathrm{G}_{1}\right) \quad T_{\text {Max }}=+\infty$ (existence of a global solution);

$\left(\mathrm{G}_{2}\right) \quad T_{M a x}<+\infty$. In this case $\lim _{T \rightarrow+T_{M a x}}\|u\|_{C([0, T], X)}=+\infty$ (blow-up in finite time).

Moreover, for all $T, 0<T<T_{\text {Max }}$, the restriction of $u$ to $[0, T]$ satisfies assertions $\left(\mathrm{L}_{1}\right)$ and $\left(\mathrm{L}_{2}\right)$, and furthermore satisfies $\left.\left(\mathrm{L}_{3}\right)\right]$ when $G:\left[0, T_{\text {Max }}\right) \rightarrow X$ defined by $G(t)=F(t, u(t))$ belongs to $W^{1,1}(0, T, X)$ for all $T, 0<T<T_{M a x}$.

Proof. We assume that $T_{M a x}<+\infty$ and show that $\lim _{T \rightarrow+T_{M a x}}\|u\|_{C([0, T], X)}=+\infty$. We argue by contradiction. Assume that $u$ does not fulfill $\lim _{T \rightarrow+T_{M a x}}\|u\|_{C([0, T], X)}=+\infty$, then there exist $\mathcal{G}>0$ and a sequence $\left(T_{n}\right)_{n \in \mathbb{N}}$ in $E$ such that $T_{n} \rightarrow T_{M a x}$ and $\|u\|_{C\left(\left[0, T_{n}\right], X\right)} \leq \mathcal{G}$.

Step 1. We show that $\lim _{t \rightarrow T_{M a x}} u(t)$ exists in $X$.

Let $n \in \mathbb{N}$. For a.e. $t \in\left(0, T_{n}\right)$ we have

$$
\left\langle\frac{d u}{d t}(t), \frac{d u}{d t}(t)\right\rangle+\left\langle D \Phi(u(t)), \frac{d u}{d t}(t)\right\rangle=\left\langle F(t, u(t)), \frac{d u}{d t}(t)\right\rangle .
$$

By integration over $\left(\delta, T_{n}\right)$ we obtain

$$
\int_{\delta}^{T_{n}}\left\|\frac{d u}{d t}(t)\right\|_{X}^{2} d t+\Phi\left(u\left(T_{n}\right)\right)-\Phi(u(\delta)) \leq\left(\int_{0}^{T_{n}}\|F(t, u(t))\|_{X}^{2} d t\right)^{\frac{1}{2}}\left(\int_{\delta}^{T_{n}}\left\|\frac{d u}{d t}(t)\right\|_{X}^{2} d t\right)^{\frac{1}{2}} .
$$

On the other hand, from $\left(C_{1}\right)$ we infer that

$$
\|F(t, u(t))\|_{X} \leq\|F(t, 0)\|_{X}+L(t)\|u(t)\|_{X},
$$

so that for a.e. $t \in(0, T)$

$$
\|F(t, u(t))\|_{X}^{2} \leq 2\|F(t, 0)\|_{X}^{2}+2 L^{2}(t)\|u\|_{C([0, T], X)}^{2} .
$$

Hence we have

$$
\begin{aligned}
\int_{0}^{T_{n}}\|F(t, u(t))\|_{X}^{2} d t & \leq 2 \int_{0}^{T_{M a x}}\|F(t, 0)\|_{X}^{2} d t+2\|u\|_{C\left(\left[0, T_{n}\right], X\right)}^{2} \int_{0}^{T_{M a x}} L^{2}(t) d t \\
& \leq 2 \int_{0}^{T_{M a x}}\|F(t, 0)\|_{X}^{2} d t+2 \mathcal{G}^{2} \int_{0}^{T_{M a x}} L^{2}(t) d t .
\end{aligned}
$$

From (1), 22), and since inf $\Phi>-\infty$, we infer that there exists a constant $C\left(\Phi, \delta, T_{M a x}, \mathcal{G}\right)$ which does not depend on $T_{n}$ such that

$$
\int_{\delta}^{T_{n}}\left\|\frac{d u}{d t}(t)\right\|_{X}^{2} d t \leq C\left(\Phi, \delta, T_{M a x}, \mathcal{G}\right)\left(1+\left(\int_{\delta}^{T_{n}}\left\|\frac{d u}{d t}(t)\right\|_{X}^{2} d t\right)^{\frac{1}{2}}\right)
$$

from which we deduce that

$$
\sup _{n \in \mathbb{N}} \int_{\delta}^{T_{n}}\left\|\frac{d u}{d t}(t)\right\|_{X}^{2} d t<+\infty
$$


From (3), we deduce that $u:\left[\delta, T_{\text {Max }}\right) \rightarrow X$ is uniformly continuous. Indeed, let $s<t$ in $\left[\delta, T_{\text {Max }}\right)$ and choose $n$ large enough (depending on $(s, t))$ so that $s$ and $t$ belong to $\left[0, T_{n}\right]$. We have

$$
\begin{aligned}
\|u(t)-u(s)\|_{X} \leq \int_{s}^{t}\left\|\frac{d u}{d t}(\tau)\right\|_{X} d \tau & \leq(t-s)^{\frac{1}{2}}\left(\int_{\delta}^{T_{n}}\left\|\frac{d u}{d t}(t)\right\|_{X}^{2} d t\right)^{\frac{1}{2}} \\
& \leq(t-s)^{\frac{1}{2}}\left(\sup _{n \in \mathbb{N}} \int_{\delta}^{T_{n}}\left\|\frac{d u}{d t}(t)\right\|_{X}^{2} d t\right)^{\frac{1}{2}},
\end{aligned}
$$

so that $u$ is more precisely $\frac{1}{2}$-Holder continuous. According to the continuous extension principle in the complete normed space $X, u$ possesses a unique continuous extension $\bar{u}$ in $\left[\delta, T_{\text {Max }}\right]$, i.e., $\lim _{t \rightarrow T_{\text {Max }}} u(t)=$ $\bar{u}\left(T_{\text {Max }}\right)$.

Step 2. (Contradiction) Consider the Cauchy problem

$$
\left(\mathcal{P}^{\prime}\right)\left\{\begin{array}{l}
\frac{d v}{d t}(t)+D \Phi(v(t))=F(t, v(t)) \text { for a.e. } t \in(0, T) \\
v(0)=\bar{u}\left(T_{\text {Max }}\right) .
\end{array}\right.
$$

Note that $\bar{u}\left(T_{\text {Max }}\right) \in \overline{\operatorname{dom}(D \Phi)}$. Indeed, $u(t) \in \operatorname{dom}(D \Phi)$ for a.e. $t$ in $(0, T)$ and $\bar{u}\left(T_{\text {Max }}\right)=$ $\lim _{t \rightarrow T_{M a x}} u(t)$ (choose $t_{n} \rightarrow T_{M a x}$ with $t_{n}$ outside the negligible set in which $u(t) \notin \operatorname{dom}(D \Phi)$ ). Then applying Theorem 2.1. there exists $T^{* *}>0$ small enough such that $\left(\mathcal{P}^{\prime}\right)$ admits a solution $v \in C\left(\left[0, T^{* *}\right], X\right)$. Set

$$
\tilde{u}(t)=\left\{\begin{array}{l}
u(t) \text { if } t \in\left[0, T_{M a x}\right] \\
v\left(t-T_{M a x}\right) \text { if } t \in\left[T_{M a x}, T_{M a x}+T^{* *}\right] .
\end{array}\right.
$$

Then $\widetilde{u} \in C\left(\left[0, T_{M a x}+T^{* *}\right], X\right)$ is a solution of $(\mathcal{P})$. This leads to a contradiction with the maximality of $T_{\text {Max }}$.

Proposition 2.1 below provides a condition on $D \Phi$ which ensures that $(\mathcal{P})$ satisfies $\left(\mathrm{G}_{1}\right)$ More precisely

Proposition 2.1. Assume that $\langle D \Phi(v), v\rangle \geq 0$ for all $v \in \operatorname{dom}(D \Phi)$. Then $(\mathcal{P})$ admits a global solution.

Proof. According to Theorem 2.2. It suffices to prove that there is no blow-up in finite time. Assume that $T_{M a x}<+\infty$ and let $T<T_{M a x}$. Taking $u(t)$ as a test function, for a.e. $t \in(0, T)$ we have

$$
\left\langle\frac{d u}{d t}(t), u(t)\right\rangle+\langle D \Phi(u(t)), u(t)\rangle=\langle F(t, u(t)), u(t)\rangle .
$$

Hence, using the fact that $\langle D \Phi(u(t)), u(t)\rangle \geq 0$ (recall that $u(t) \in \operatorname{dom}(D \Phi)$ for a.e. $t \in(0, T)$ ), we infer that

$$
\begin{aligned}
\frac{d}{d t}\|u(t)\|_{X}^{2} & \leq 2\langle F(t, u(t)), u(t)\rangle \\
& \leq\left(\|F(t, 0)\|_{X}+L(t)\|u(t)\|_{X}\right)^{2}+\|u(t)\|_{X}^{2} \\
& \leq 2\|F(t, 0)\|_{X}^{2}+\left(2 L^{2}(t)+1\right)\|u(t)\|_{X}^{2} .
\end{aligned}
$$

By integrating over $(0, s)$ for $s \in[0, T]$, we deduce

$$
\|u(s)\|_{X}^{2} \leq\left\|u_{0}\right\|_{X}^{2}+2 \int_{0}^{s}\|F(t, 0)\|_{X}^{2} d t+\int_{0}^{s}\left(2 L^{2}(t)+1\right)\|u(t)\|_{X}^{2} d t
$$

(note that from $\left(\mathrm{C}_{2}\right), t \mapsto\|F(t, 0)\|_{X}^{2}$ belongs to $L^{1}(0, T)$, and $t \mapsto\left(2 L^{2}(t)+1\right)\|u(t)\|_{X}^{2}$ belongs to $L^{1}(0, T)$ since $\|u(t)\|_{X} \leq\|u\|_{C([0, T], X)}$ and $\left.L \in L^{2}(0, T)\right)$. By using Gronwall's lemma and the continuity in $[0, T]$ of

$$
s \mapsto\left\|u_{0}\right\|_{X}^{2}+2 \int_{0}^{s}\|F(t, 0)\|_{X}^{2} d t+\int_{0}^{s}\left(2 L^{2}(t)+1\right)\|u(t)\|_{X}^{2} d t-\|u(s)\|_{X}^{2},
$$


we obatin for all $t \in[0, T]$ :

$$
\|u(t)\|_{X}^{2} \leq\left(\left\|u_{0}\right\|_{X}^{2}+2 \int_{0}^{t}\|F(s, 0)\|_{X}^{2} d s\right) \exp \left(\int_{0}^{t}\left(2 L^{2}(s)+1\right) d s\right) .
$$

Then, if $T_{M a x}<+\infty$, we have

$$
\sup _{T<T_{\text {Max }}}\|u\|_{C([0, T], X)} \leq\left(\left\|u_{0}\right\|_{X}^{2}+2 \int_{0}^{T_{M a x}}\|F(s, 0)\|_{X}^{2} d s\right) \exp \left(\int_{0}^{T_{M a x}}\left(2 L^{2}(s)+1\right) d s\right) .
$$

This makes $\lim _{T \rightarrow+T_{M a x}}\|u\|_{C([0, T], X)}=+\infty$ impossible. Thus $T_{M a x}=+\infty$.

From Proposition 2.1. when $\Phi=0,\left(\mathrm{G}_{1}\right)$ is automatically satisfied. Therefore we obtain the following global existence for non diffusive problems

Theorem 2.3 (Global existence for non diffusive Cauchy problems). Assume that $F$ satisfies $\left(\mathrm{C}_{1}\right),\left(\mathrm{C}_{2}\right)$, Then, there exists a unique global solution $u \in C([0,+\infty), X)$ of the non diffusive Cauchy problem

$$
(\mathcal{P})\left\{\begin{array}{l}
\frac{d u}{d t}(t)=F(t, u(t)) \text { for a.e. } t \in(0, T) \\
u(0)=u_{0}, u_{0} \in X .
\end{array}\right.
$$

Moreover, for all $T<T_{M a x}$ the restriction of $u$ to $[0, T]$ satisfies assertions $\left(\mathrm{L}_{1}\right)$ and $\left(\mathrm{L}_{2}\right)$, and furthermore sitisfies $\left(\mathrm{L}_{3}\right)$ when $G:\left[0, T_{\text {Max }}\right) \rightarrow X$ defined by $G(t)=F(t, u(t))$ belongs to $W^{1,1}(0, T, X)$.

\section{EXISTENCE AND UNIQUENESS OF BOUNDED SOLUTION FOR A CLASS OF REACTION-DIFFUSION} PROBLEMS

From now on $\Omega$ is a domain of $\mathbb{R}^{N}$ of class $C^{1}$ and $\mathcal{L}_{N}$ denotes the Lebesgue measure on $\mathbb{R}^{N}$. We denote by $\partial \Omega$ its boundary and by $\mathcal{H}_{N-1}$ the $N$-1-dimensional Hausdorff measure. To shorten the notation, we sometimes write $X$ to denote the Hilbert space $L^{2}(\Omega)$ equipped with its standard scalar product and its associated norm, denoted by $\langle\cdot, \cdot\rangle$ and $\|\cdot\|_{X}$ respectively.

3.1. The class of diffusion terms associated with convex functionals of the calculus of variations. In all the paper, we focus on the specific case of a standard convex functional $\Phi$ of the calculus of variations, i.e., a functional $\Phi: L^{2}(\Omega) \rightarrow \mathbb{R} \cup\{+\infty\}$ defined by

$$
\Phi(u)=\left\{\begin{array}{cl}
\int_{\Omega} W(x, \nabla u(x)) d x+\frac{1}{2} \int_{\partial \Omega} a_{0} u^{2} d \mathcal{H}_{N-1}-\int_{\partial \Omega} h u d \mathcal{H}_{N-1} & \text { if } u \in H^{1}(\Omega) \\
+\infty & \text { otherwise }
\end{array}\right.
$$

wher $\AA^{2} h \in L_{\mathcal{H}_{N-1}}^{2}(\partial \Omega), a_{0} \in L_{\mathcal{H}_{N-1}}^{\infty}(\partial \Omega)$ with $a_{0} \geq 0 \mathcal{H}_{N-1}$-a.e. in $\partial \Omega$ and $a_{0} \geq \sigma$ on $\Gamma \subset \partial \Omega$ with $\mathcal{H}_{N-1}(\Gamma)>0$ for some $\sigma>0$.

The density $W: \mathbb{R}^{N} \times \mathbb{R}^{N} \rightarrow \mathbb{R}$ is a Borel measurable function which satisfies the following conditions:

$\left(\mathrm{D}_{1}\right)$ there exist $\alpha>0$ and $\beta>0$ such that for a.e. $x \in \mathbb{R}^{N}$ and every $\xi \in \mathbb{R}^{N}$

$$
\alpha|\xi|^{2} \leq W(x, \xi) \leq \beta\left(1+|\xi|^{2}\right),
$$

$\left(\mathrm{D}_{2}\right)$ for a.e. $x \in \mathbb{R}^{N}, \xi \mapsto W(x, \xi)$ is a Gâteaux differentiable and convex function (we denote by $D_{\xi} W(x, \cdot)$ its Gâteaux derivative), and

$$
D_{\xi} W(x, 0)=0 .
$$

By using the subdifferential inequality together with the growth conditions $\left(\mathrm{D}_{1}\right)$, it is easy to show that there exist nonnegative constants $L(\beta)$ and $C(\beta)$ such that, for all $\left(\xi, \xi^{\prime}\right) \in \mathbb{R}^{N} \times \mathbb{R}^{N}$,

$$
\left\{\begin{array}{l}
\left|W(x, \xi)-W\left(x, \xi^{\prime}\right)\right| \leq L(\beta)\left|\xi-\xi^{\prime}\right|\left(1+|\xi|+\left|\xi^{\prime}\right|\right), \\
\left|D_{\xi} W(x, \xi)\right| \leq C(\beta)(1+|\xi|) .
\end{array}\right.
$$

From the second estimate, we infer that if $u \in H^{1}(\Omega)$, then the function $D_{\xi} W(\cdot, \nabla u)$ belongs to $L^{2}(\Omega)^{N}$.

\footnotetext{
${ }^{2}$ In the integrals on $\partial \Omega$, we still denote by $u$ the trace of $u$.
} 
Remark 3.1. We do not loss of generality by considering $D_{\xi} W(x, 0)=0$ in $\left(\mathrm{D}_{2}\right)$. Indeed, for any Borel measurable function $W: \mathbb{R}^{N} \times \mathbb{R}^{N} \rightarrow \mathbb{R}$ satisfying $\left(\mathrm{D}_{1}\right)$ with $\xi \mapsto W(x, \xi)$ Gâteaux differentiable and convex, define the function $\widetilde{W}$ by

$$
\widetilde{W}(x, \xi)=W(x, \xi)-D_{\xi} W(x, 0) \cdot \xi+C(\beta)|\xi|^{2} .
$$

Then $\xi \mapsto \widetilde{W}(x, \xi)$ is convex, Gâteaux differentiable with $D_{\xi} \widetilde{W}(x, 0)=0$. Moreover $\widetilde{W}$ satisfies the upper growth condition of $\left(\mathrm{D}_{1}\right)$ and the lower growth condition up to an additive constant, with two other positive constants $\alpha^{\prime}$ and $\beta^{\prime}$.

Consider the space $\mathbf{H}(\operatorname{div}):=\left\{\sigma \in L^{2}(\Omega)^{N}: \operatorname{div} \sigma \in L^{2}(\Omega)\right\}$. It is well known that when $\Omega$ is an open domain of class $C^{1}$, with outer unit normal $\mathbf{n}$, the normal trace

$$
\gamma_{\mathbf{n}}: \mathbf{H}(\operatorname{div}) \cap C(\bar{\Omega}) \rightarrow H^{-\frac{1}{2}}(\partial \Omega) \cap C(\partial \Omega)
$$

defined by $\gamma_{\mathbf{n}}(\sigma)=(\sigma \cdot \mathbf{n})\left\lfloor_{\partial \Omega}\right.$, has a continuous extension from $\mathbf{H}(\operatorname{div})$ onto $H^{-\frac{1}{2}}(\partial \Omega)$, still denoted by $\gamma_{\mathbf{n}}$. Moreover, the following Green's formula holds: for every $\varphi \in H^{1}(\Omega)$ whose trace denoted by $\gamma_{0}(\varphi)$ belongs to $H^{\frac{1}{2}}(\partial \Omega)$, we have

$$
\int_{\Omega} \operatorname{div} \sigma \varphi d x=-\int_{\Omega} \sigma \cdot \nabla \varphi d x+\left\langle\gamma_{\mathbf{n}}(\sigma), \gamma_{0}(\varphi)\right\rangle_{H^{-\frac{1}{2}}(\partial \Omega), H^{\frac{1}{2}}(\partial \Omega)} .
$$

In all the paper, as usual, for simplicity of notation, for any $\sigma \in \mathbf{H}(\operatorname{div})$ and any $\varphi \in H^{1}(\Omega)$, we (improperly) write, $\int_{\partial \Omega} \sigma \cdot \mathbf{n} \varphi d \mathcal{H}_{N-1}$ the last term $\left\langle\gamma_{\mathbf{n}}(\sigma), \gamma_{0}(\varphi)\right\rangle_{H^{-\frac{1}{2}}(\partial \Omega), H^{\frac{1}{2}}(\partial \Omega)}$, and, as for regular functions, we denote by $\sigma \cdot \mathbf{n}$ and $\varphi$ the normal trace and the trace of $\sigma$ and $\varphi$ respectively. We start by expliciting the subdifferential of the functional $\Phi$ (actually its Gâteaux derivative), whose domain contains mixed Dirichlet-Neumann boundary conditions. For a detailed proof we refer the reader to 8 , Theorem 17.2.10] where $\Phi$ is a more basic integral functional.

Lemma 3.1. The subdifferential of the functional $\Phi$ is the operator $A=\partial \Phi(=D \Phi)$ defined by

$$
\left\{\begin{array}{l}
\operatorname{dom}(A)=\left\{v \in H^{1}(\Omega): \operatorname{div} D_{\xi} W(\cdot, \nabla v) \in L^{2}(\Omega), a_{0} v+D_{\xi} W(\cdot, \nabla v) \cdot \mathbf{n}=h \text { on } \partial \Omega\right\} \\
A(v)=-\operatorname{div} D_{\xi} W(\cdot, \nabla v) \text { for } v \in \operatorname{dom}(A)
\end{array}\right.
$$

where $a_{0} v+D_{\xi} W(\cdot, \nabla v) \cdot \mathbf{n}$ must be taken in the trace sense.

Assume that $h=0$. Let $\Gamma$ be a subset of $\partial \Omega$ with $\mathcal{H}_{N-1}(\Gamma)>0$ and define $a_{0}$ in $[0,+\infty]$ in the following way:

$$
a_{0}(x)= \begin{cases}0 & \text { if } x \in \partial \Omega \backslash \Gamma \\ +\infty & \text { if } x \in \Gamma .\end{cases}
$$

Then, the integral $\int_{\partial \Omega} a_{0} u^{2} d \mathcal{H}_{N-1}$ may be considered as a penalization which forces the function $u$ to belong to $H_{\Gamma}^{1}(\Omega)=\left\{u \in H^{1}(\Omega): u=0\right.$ on $\left.\Gamma\right\}$. By convention the functional $\Phi$ becomes

$$
\Phi(u)=\left\{\begin{array}{cl}
\int_{\Omega} W(x, \nabla u(x)) d x & \text { if } u \in H_{\Gamma}^{1}(\Omega) \\
+\infty & \text { otherwise. }
\end{array}\right.
$$

The subdifferential of $\Phi$ contains now the homogeneous Dirichlet-Neumann boundary conditions as stated in the following lemma which can be proved by an easy adaptation of the proof of Lemma 3.1 .

Lemma 3.2. The subdifferential of the functional $\Phi$ is the operator $A=\partial \Phi(=D \Phi)$ defined by

$$
\left\{\begin{array}{l}
\operatorname{dom}(A)=\left\{v \in H_{\Gamma}^{1}(\Omega): \operatorname{div} D_{\xi} W(\cdot, \nabla v) \in L^{2}(\Omega), D_{\xi} W(\cdot, \nabla v) \cdot \mathbf{n}=0 \text { on } \partial \Omega \backslash \Gamma\right\} \\
A(v)=-\operatorname{div} D_{\xi} W(\cdot, \nabla v) \text { for } v \in \operatorname{dom}(A) .
\end{array}\right.
$$


3.2. The class of CP-structured reaction functionals. The reaction-diffusion problems modeling a wide variety of applications, and amenable to analytical manipulation in homogenization (periodic or stochastic), involve a special class of functionals that we define below.

Definition 3.1. A map $F:[0,+\infty) \times L^{2}(\Omega) \rightarrow \mathbb{R}^{\Omega}$ is called a CP-structured reaction functional, if there exists a Borel measurable function $f:[0,+\infty) \times \mathbb{R}^{N} \times \mathbb{R} \rightarrow \mathbb{R}$ such that for all $t \in[0,+\infty)$ and all $v \in L^{2}(\Omega), F(t, v)(x)=f(t, x, v(x))$, and fulfills the following structure conditions:

$$
f(t, x, \zeta)=r(t, x) \cdot g(\zeta)+q(t, x)
$$

with

- $g: \mathbb{R} \rightarrow \mathbb{R}^{l}$ is a locally Lipschitz continuous function;

- for all $T>0, r$ belongs to $L^{\infty}\left([0, T] \times \mathbb{R}^{N}, \mathbb{R}^{l}\right)$;

- for all $T>0, q$ belongs to $L^{2}\left(0, T, L_{\text {loc }}^{2}\left(\mathbb{R}^{N}\right)\right)$.

Furthermore $f$ must satisfy the following condition:

(CP) there exist a pair $(\underline{f}, \bar{f})$ of functions $\underline{f}, \bar{f}:[0,+\infty) \times \mathbb{R} \rightarrow \mathbb{R}$ with $\underline{f} \leq 0 \leq \bar{f}$ and a pair $(\underline{\rho}, \bar{\rho})$ in $\mathbb{R}^{2}$ with $\rho \leq \bar{\rho}$, such that each of the two following ordinary differential equations

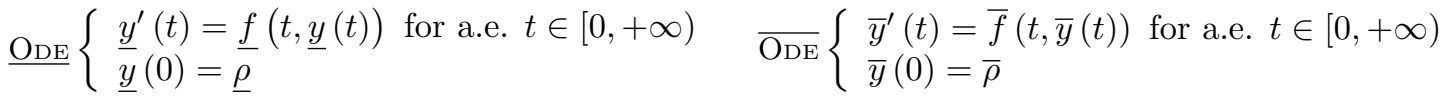

admits at least one solution denoted by $\underline{y}$ for $\underline{\mathrm{ODE}_{\mathrm{DE}}}$ and by $\bar{y}$ for $\overline{\mathrm{ODE}}$ satisfying for a.e. $(t, x) \in$ $(0,+\infty) \times \mathbb{R}$

$$
\underline{f}(t, \underline{y}(t)) \leq f(t, x, \underline{y}(t)) \quad \text { and } \quad f(t, x, \bar{y}(t)) \leq \bar{f}(t, \bar{y}(t)) .
$$

The map $F$ is referred to as a CP-structured reaction functional associated with $(r, g, q)$, and $f$ as a $C P$ structured reaction function associated with $(r, g, q)$. The map $F$ is referred to as a regular CP-structured reaction functional and $f$ as a regular CP-structured reaction function if furthermore, for all $T>0$, $r \in W^{1,1}\left(0, T, L_{\text {loc }}^{2}\left(\mathbb{R}^{N}, \mathbb{R}^{l}\right)\right)$ and $q \in W^{1,1}\left(0, T, L_{\text {loc }}^{2}\left(\mathbb{R}^{N}\right)\right)$.

Remark 3.2. 1) Since $y$ and $\bar{y}$ are nonincreasing and nondecreasing respectively, for any $T>0$ we have $\underline{y}(T) \leq \underline{y}(0)=\underline{\rho} \leq \bar{\rho}=\bar{y}(0) \leq \bar{y}(T)$.

2) We introduce the spaces $L_{\text {loc }}^{2}\left(\mathbb{R}^{N}\right)$ and $L_{\text {loc }}^{2}\left(\mathbb{R}^{N}, \mathbb{R}^{l}\right)$ because of the specific form of sequences of CP-structured reaction functionals $F_{\varepsilon}$ in the framework of homogenization where the scaling $x \mapsto \frac{x}{\varepsilon}$ appears. Nevertheless, in Section 3 , we can replace these two spaces by $L^{2}(\Omega)$ and $L^{2}\left(\Omega, \mathbb{R}^{l}\right)$ respectively. Note that when $X$ is a reflexive space, $W^{1,1}(0, T, X)$ is exactly the space of absolutely functions from $[0, T]$ into $X$ (see [13, Corollary A4]).

3) The reason why we introduce condition (CP) may be summarized as follows: even if CP-structured reaction functionals do not satisfy the Lipschitz condition $\left(\mathrm{C}_{1}\right)$ invoked in Theorems 2.1, 2.2. according to the comparison principle (Proposition 3.1 and Proposition 3.2 below), we can prove that reaction-diffusion problems associated with a CP-structured reaction functional admits a unique solution which satisfies $\underline{y}(T) \leq u \leq \bar{y}(T)$ whenever the initial condition satisfies $\underline{\rho} \leq$ $u_{0} \leq \bar{\rho}$ (see Section 3.4).

3.3. The comparison principle. Let us set $V:=\left\{v \in H^{1}(\Omega): \operatorname{div} D_{\xi} W(\cdot, \nabla v) \in L^{2}(\Omega)\right\}$, and consider two functionals $F_{1}, F_{2}:[0,+\infty) \times L^{2}(\Omega) \rightarrow L^{2}(\Omega)$ defined by

$$
F_{1}(t, u)(x)=f_{1}(t, x, u(x)), \quad F_{2}(t, u)(x)=f_{2}(t, x, u(x))
$$

where $f_{1}, f_{2}:[0,+\infty) \times \mathbb{R}^{N} \times \mathbb{R} \rightarrow \mathbb{R}$ are two measurable functions, $f_{2}$ being Lipschitz continuous uniformly with respect to $(t, x)$, i.e., fulfills the condition $\left|f_{2}(t, x, \zeta)-f_{2}\left(t, x, \zeta^{\prime}\right)\right| \leq L\left|\zeta-\zeta^{\prime}\right|$. Moreover, we are given two functions $u_{0}$ and $v_{0}$ in $H^{1}(\Omega)$ and two functions $h_{1}$ and $h_{2}$ in $L^{2}\left(0, T, L_{\mathcal{H}_{N-1}}^{2}(\partial \Omega)\right)$. The following comparison result will be used for proving existence of bounded solutions of reactiondiffusion problems associated with special reaction functionals (see Subsection 3.2). For similar notion and applications of sub and supersolution related to elliptic boundary valued problems we refer the reader to [10, 11] and for parabolic problems, to [21]. 
Proposition 3.1. Let $T>0, u \in C\left([0, T], L^{2}(\Omega)\right)$ and $v \in C\left([0, T], L^{2}(\Omega)\right)$ be a subsolution and a supersolution of the reaction-diffusion problems with respect to the data $\left(u_{0}, h_{1}, F_{1}\right)$ and $\left(v_{0}, h_{2}, F_{2}\right)$, i.e.,

$$
\begin{aligned}
& \frac{\mathcal{P}\left(u_{0}, h_{1}, F_{1}\right)}{\quad}\left\{\begin{array}{l}
u(t) \in V, \frac{d u}{d t}(t) \in L^{2}(\Omega) \text { for a.e. } t \in(0, T), \\
u(0)=u_{0} \in L^{2}(\Omega), \\
a_{0} u(t)+D_{\xi} W(\cdot, \nabla u(t)) \cdot \mathbf{n}=h_{1}(t) \text { on } \partial \Omega \text { for a.e. } t \in(0, T),
\end{array}\right. \\
& \overline{\mathcal{P}\left(v_{0}, h_{2}, F_{2}\right)}\left\{\begin{array}{l}
v(t) \in V, \frac{d v}{d t}(t) \in L^{2}(\Omega) \text { for a.e. } t \in(0, T), \\
\frac{d v}{d t}(t)-\operatorname{div} D_{\xi} W(\cdot, \nabla v(t)) \geq F_{2}(t, v(t)) \text { for a.e. } t \in(0, T), \\
v(0)=v_{0} \in L^{2}(\Omega), \\
a_{0} v(t)+D_{\xi} W(\cdot, \nabla v(t)) \cdot \mathbf{n}=h_{2}(t) \text { on } \partial \Omega \text { for a.e. } t \in(0, T) .
\end{array}\right.
\end{aligned}
$$

Then the following comparison principle holds:

$$
\left.\begin{array}{l}
u_{0} \leq v_{0} \text { in } L^{2}(\Omega), \\
h_{1}(t) \leq h_{2}(t) \text { on } \partial \Omega, \text { for a.e. } t \in(0, T), \\
F_{1} \leq F_{2}
\end{array}\right\} \Longrightarrow u(t) \leq v(t) \text { for all } t \in[0, T] .
$$

Proof. Set $w=v-u$. We are going to prove that $w(t)^{-}=0$ for a.e. $t \in(0, T)$. Indeed, for a.e. $t \in(0, T)$ we have

$$
\frac{d w}{d t}(t)-\left[\operatorname{div} D_{\xi} W(\cdot, \nabla v(t))-\operatorname{div} D_{\xi} W(\cdot, \nabla u(t))\right] \geq F_{2}(t, v(t))-F_{1}(t, u(t)) .
$$

Take $w(t)^{-}$as a test function. By integrating over $\Omega$, and using Green's formula we obtain

$$
\begin{aligned}
\int_{\Omega} \frac{d w}{d t}(t) w(t)^{-} d x & +\int_{\Omega}\left(D_{\xi} W(x, \nabla v(t))-D_{\xi} W(x, \nabla u(t))\right) \cdot \nabla w(t)^{-} d x \\
& -\int_{\partial \Omega}\left(D_{\xi} W(x, \nabla v(t))-D_{\xi} W(x, \nabla u(t))\right) \cdot \mathbf{n} w(t)^{-} d \mathcal{H}_{N-1} \\
& \geq \int_{\Omega}\left(f_{2}(t, x, v(t))-f_{1}(t, x, u(t))\right) w(t)^{-} d x .
\end{aligned}
$$

Noticing that $D_{\xi} W(x, \nabla u(t)) \cdot \mathbf{n}=h_{1}(t)-a_{0} u(t)$, and $D_{\xi} W(x, v(t)) \cdot \mathbf{n}=h_{2}(t)-a_{0} v(t)$ on $\partial \Omega$, we infer that

$$
\begin{aligned}
\int_{\Omega} \frac{d w}{d t}(t) w(t)^{-} d x & +\int_{\Omega}\left(D_{\xi} W(x, \nabla v(t))-D_{\xi} W(x, u(t))\right) \cdot \nabla w(t)^{-} d x \\
& +\int_{\partial \Omega}\left(h_{1}(t)-h_{2}(t)\right) w(t)^{-} d \mathcal{H}_{N-1}+\int_{\partial \Omega} a_{0}(v-u) w^{-} d \mathcal{H}_{N-1} \\
& \geq \int_{\Omega}\left(f_{2}(t, x, v(t))-f_{1}(t, x, u(t))\right) w(t)^{-} d x
\end{aligned}
$$

from which we deduce

$$
\begin{aligned}
-\int_{\Omega} \frac{d w^{-}}{d t}(t) w(t)^{-} d x & -\int_{[w(t) \leq 0]}\left(D_{\xi} W(x, \nabla v(t))-D_{\xi} W(x, u(t))\right) \cdot(\nabla v(t)-\nabla u(t)) d x \\
& -\int_{\partial \Omega}\left(h_{2}(t)-h_{1}(t)\right) w(t)^{-} d \mathcal{H}_{N-1}-\int_{[w(t) \leq 0] \cap \partial \Omega} a_{0}(v-u)^{2} d \mathcal{H}_{N-1} \\
\geq & \int_{\Omega}\left(f_{2}(t, x, v(t))-f_{1}(t, x, u(t))\right) w(t)^{-} d x,
\end{aligned}
$$


where we have used the relations

$$
\left(\frac{d w}{d t}(t)\right)^{+}=\frac{d w^{+}}{d t}(t),\left(\frac{d w}{d t}(t)\right)^{-}=\frac{d w^{-}}{d t}(t), \frac{d w}{d t}(t)=\frac{d w^{+}}{d t}(t)-\frac{d w^{-}}{d t}(t) \text { and } \frac{d w^{+}}{d t}(t) w(t)^{-}=0
$$

in the distributional sense. Noticing that the three last integrands of the first member are nonnegative, and $f_{1} \leq f_{2}$, we obtain

$$
\frac{1}{2} \frac{d}{d t} \int_{\Omega}\left|w(t)^{-}\right|^{2} d x \leq \int_{\Omega}\left(f_{2}(t, x, u(t))-f_{2}(t, x, v(t))\right) w(t)^{-} d x .
$$

From (6) and the Lipschitz continuity of the function $f_{2}$, we deduce that

$$
\frac{1}{2} \frac{d}{d t} \int_{\Omega}\left|w(t)^{-}\right|^{2} d x \leq L \int_{\Omega}|w(t)| w(t)^{-} d x=L \int_{\Omega}\left|w(t)^{-}\right|^{2} d x .
$$

Integrating this inequality over $(0, s)$ for $s \in[0, T]$, we obtain

$$
\int_{\Omega}\left|w(s)^{-}\right|^{2} d x-\int_{\Omega}\left|w(0)^{-}\right|^{2} d x \leq 2 L \int_{0}^{s}\left(\int_{\Omega}\left|w(t)^{-}\right|^{2} d x\right) d t .
$$

Note that since $w^{-} \in C\left([0, T], L^{2}(\Omega)\right), s \mapsto \int_{\Omega}\left|w(s)^{-}\right|^{2} d x$ is continuous. Then, according to Gronwall's lemma we finally obtain that for all $s \in[0, T]$

$$
\int_{\Omega}\left|w(s)^{-}\right|^{2} d x \leq \int_{\Omega}\left|w(0)^{-}\right|^{2} d x \exp (2 L s)
$$

from which we deduce, since $w(0)^{-}=\left(v_{0}-u_{0}\right)^{-}=0$, that $w(s)^{-}=0$ in $L^{2}(\Omega)$ for all $s \in[0, T]$, i.e., $u(s) \leq v(s)$ for all $s \in[0, T]$.

Let us consider the case

$$
a_{0}(x)= \begin{cases}0 & \text { if } x \in \partial \Omega \backslash \Gamma \\ +\infty & \text { if } x \in \Gamma\end{cases}
$$

with $h=0$, and set $\widetilde{V}:=\left\{v \in H^{1}(\Omega): \operatorname{div} D_{\xi} W(\cdot, \nabla v) \in L^{2}(\Omega), D_{\xi} W(\cdot, \nabla v) \cdot \mathbf{n}=0\right.$ on $\left.\partial \Omega \backslash \Gamma\right\}$. Then an easy adaptation of the previous proof leads to the following comparison principle

Proposition 3.2. Let $T>0, u \in C\left([0, T], L^{2}(\Omega)\right)$ and $v \in C\left([0, T], L^{2}(\Omega)\right)$ be a subsolution and a supersolution of the reaction-diffusion problems with respect to the data $\left(u_{0}, F_{1}\right)$ and $\left(v_{0}, F_{2}\right)$, i.e.,

$$
\begin{aligned}
& \frac{\mathcal{P}\left(u_{0}, h_{1}, F_{1}\right)}{\quad}\left\{\begin{array}{l}
u(t) \in \widetilde{V}, \frac{d u}{d t}(t) \in L^{2}(\Omega) \text { for a.e. } t \in(0, T), \\
\frac{d u}{d t}(t)-\operatorname{div} D_{\xi} W(\cdot, \nabla u(t)) \leq F_{1}(t, u(t)) \text { for a.e. } t \in(0, T), \\
u(0)=u_{0} \in L^{2}(\Omega),
\end{array}\right. \\
& \overline{\mathcal{P}\left(v_{0}, h_{2}, F_{2}\right)}\left\{\begin{array}{l}
v(t) \in \widetilde{V}, \frac{d v}{d t}(t) \in L^{2}(\Omega) \text { for a.e. } t \in(0, T), \\
\frac{d v}{d t}(t)-\operatorname{div} D_{\xi} W(\cdot, \nabla v(t)) \geq F_{2}(t, v(t)) \text { for a.e. } t \in(0, T), \\
v(0)=v_{0} \in L^{2}(\Omega) .
\end{array}\right.
\end{aligned}
$$

Then the following comparison principle holds:

$$
\left.\begin{array}{l}
u_{0} \leq v_{0} \text { in } L^{2}(\Omega), \\
u(t) \leq v(t) \text { on } \Gamma, \text { for a.e. } t \in(0, T), \\
F_{1} \leq F_{2}
\end{array}\right\} \Longrightarrow u(t) \leq v(t) \text { for all } t \in[0, T]
$$


3.4. Existence and uniqueness of a bounded solution. Combining Theorems 2.1 2.2 with the comparison principle we can establish the existence of a bounded solution of the Cauchy problem associated with CP-structured reaction functionals.

Theorem 3.1. Let $F$ be a CP-structured reaction functional, with $\underline{\rho}, \bar{\rho}$ and $\underline{y}, \bar{y}$ given by $(\mathrm{CP})$, and let $\Phi$ be a standard functional of the calculus of variations (4) of Section 3.1 Assume that $a_{0} \underline{\rho} \leq h \leq a_{0} \bar{\rho}$ on $\partial \Omega$. Then for any $T>0$, the Cauchy problem

$$
(\mathcal{P})\left\{\begin{array}{l}
\frac{d u}{d t}(t)+D \Phi(u(t))=F(t, u(t)) \text { for a.e. } t \in(0, T) \\
u(0)=u_{0}, \quad \underline{\rho} \leq u_{0} \leq \bar{\rho}, \quad u_{0} \in \overline{\operatorname{dom}(D \Phi)}
\end{array}\right.
$$

admits a unique solution $u \in C\left([0, T], L^{2}(\Omega)\right)$ satisfying assertions $\left(\mathrm{L}_{1}\right),\left(\mathrm{L}_{2}\right)$ and the following bounds in $[0, T]: y(T) \leq y(t) \leq u(t) \leq \bar{y}(t) \leq \bar{y}(T)$. If furthermore $F$ is a regular CP-structured reaction functional, then $u$ satisfies $\left(\mathrm{L}_{3}\right)$

Proof. The proof of $\inf _{v \in L^{2}(\Omega)} \Phi(v)>-\infty$ is obtained from a standard calculation (see [?]).

Step 1. We prove existence of a solution $u$ of $(\mathcal{P})$ for $T=T^{*}>0$ small enough, which satisfies $\left(\mathrm{L}_{1}\right),\left(\mathrm{L}_{2}\right)$ and the bounds $\underline{y}\left(T^{*}\right) \leq \underline{y}(t) \leq u(t) \leq \bar{y}(t) \leq \bar{y}\left(T^{*}\right)$.

By definition of CP-structured reaction functionals, $F:[0,+\infty) \times L^{2}(\Omega) \rightarrow \mathbb{R}^{\Omega}$ is defined for all $t \in[0,+\infty)$, all $v \in L^{2}(\Omega)$, and for a.e. $x \in \Omega$ by $F(t, v)(x)=f(t, x, v(x))$, where for all $\zeta \in \mathbb{R}$

$$
f(t, x, \zeta)=r(t, x) \cdot g(\zeta)+q(t, x),
$$

and where $g: \mathbb{R} \rightarrow \mathbb{R}^{l}$ is locally Lipschitz continuous. Fix arbitrary $T^{\prime}>0$. The restriction of $g$ to the interval $\left[y\left(T^{\prime}\right), \bar{y}\left(T^{\prime}\right)\right]$ is Lipschitz continuous with some lipschitz constant $L_{g}{ }^{3}$ Consequently $\zeta \mapsto f(t, x, \zeta)$ is Lipschitz continuous with respect to $\zeta$, uniformly with respect to $(t, x)$ in $\left[\underline{y}\left(T^{\prime}\right), \bar{y}\left(T^{\prime}\right)\right]$, with

$$
\left|f(t, x, \zeta)-f\left(t, x, \zeta^{\prime}\right)\right| \leq L\left|\zeta-\zeta^{\prime}\right|
$$

where $L=L_{g}\|r\|_{L^{\infty}\left(\left[0, T^{\prime}\right] \times \mathbb{R}^{N}, \mathbb{R}^{l}\right)}$. According to the Mac Shane extension lemma, $g$ can be extended into a Lipschitz continuous function $\widetilde{g}$ in $\mathbb{R}$. Hence the extension $\tilde{f}$ of $f$ defined by $\widetilde{f}(t, x, \zeta)=r(t, x)$. $\widetilde{g}(\zeta)+q(t, x)$ is Lipschitz continuous with respect to $\zeta$ in $\mathbb{R}$, uniformly with respect to $(t, x)$, with the same Lipschitz constant $L$. Consequently, the functional $\widetilde{F}:[0,+\infty) \times L^{2}(\Omega) \rightarrow L^{2}(\Omega)$ defined by $\widetilde{F}(t, v)(x)=\widetilde{f}(t, x, v(x))$ satisfies $\left(\mathrm{C}_{1}\right)$ and $\left(\mathrm{C}_{2}\right)$ with $L(t)=L$.

Therefore, according to Theorem 2.1. for $T^{*}>0$ small enough, that we can choose such that $T^{*} \leq T^{\prime}$, the problem

$$
(\widetilde{\mathcal{P}})\left\{\begin{array}{l}
\frac{d \widetilde{u}}{d t}(t)+D \Phi(\widetilde{u}(t))=\widetilde{F}(t, \widetilde{u}(t)) \text { for a.e. } t \in\left(0, T^{*}\right) \\
\widetilde{u}(0)=u_{0}
\end{array}\right.
$$

admits a unique solution in $C\left(\left[0, T^{*}\right], X\right)$ which satisfies $\left(\mathrm{L}_{1}\right)$ and $\left(\mathrm{L}_{2}\right)$.

By using Proposition 3.1. we are going to prove that for all $t \in\left[0, T^{*}\right], \widetilde{u}(t) \in[\underline{y}(t), \bar{y}(t)] \subset$ $\left[\underline{y}\left(T^{*}\right), \bar{y}\left(T^{*}\right)\right]$. From condition (CP), the function $y$, which does not depend on $x$, is a subsolution of the reaction-diffusion problem $\widetilde{\mathcal{P}}\left(\underline{\rho}, a_{0} \underline{y}(t), \widetilde{F}\right)$ in the sense of Proposition 3.1 . Indeed since $\underline{y}(t) \in\left[\underline{y}\left(T^{\prime}\right), \bar{y}\left(T^{\prime}\right)\right], D_{\xi} W(x, 0)=0, \overline{\text { and } \nabla} \underline{y}=0$, we have for all $t \in\left[0, T^{*}\right]$

$$
\left\{\begin{array}{l}
\widetilde{F}(t, \underline{y}(t))=F(t, \underline{y}(t))=f(t, \cdot, \underline{y}(t)) \geq \underline{f}(t, \underline{y}(t))=\underline{y}^{\prime}(t)=\frac{d \underline{y}}{d t}(t)-\operatorname{div} D_{\xi} W(\cdot, \nabla \underline{y}(t)), \\
\text { initial condition } \underline{y}(0)=\underline{\rho}, \\
\text { boundary condition } a_{0} \underline{y}(t)+D_{\xi} W(x, \nabla \underline{y}(t)) \cdot \mathbf{n}=a_{0} \underline{y}(t) \text { on } \partial \Omega .
\end{array}\right.
$$

On the other hand $\widetilde{u}$ is a solution of $(\widetilde{\mathcal{P}})$, thus a supersolution of $\widetilde{\mathcal{P}}\left(u_{0}, \phi, \widetilde{F}\right)$. From the comparison principle, Proposition 3.1 , since $\underline{\rho} \leq u_{0}$, and $a_{0} \underline{y}(t) \leq a_{0} \underline{y}(0)=a_{0} \underline{\rho} \leq h$, we infer that $\underline{y}(t) \leq \widetilde{u}(t)$

\footnotetext{
${ }^{3}$ To simplify the notation, we do not indicate the dependance on $T^{\prime}$.
} 
for a.e. $t \in\left(0, T^{*}\right)$. Actually, inequality $\underline{y}(t) \leq \widetilde{u}(t)$ holds for all $t \in\left[0, T^{*}\right]$ (invoke the continuity of $\left.t \mapsto\left\|(\widetilde{u}(t)-\underline{y}(t))^{-}\right\|_{X}\right)$. Reasoning similarly with $\bar{y}$ which is a supersolution of $\widetilde{\mathcal{P}}\left(\bar{\rho}, a_{0} \bar{y}(t), \widetilde{F}\right)$, we obtain that $\bar{y}(t) \geq \widetilde{u}(t)$ for all $t \in[0, T]^{*}$. To sum up we have $\widetilde{u}(t) \in[\underline{y}(t), \bar{y}(t)] \subset\left[\underline{y}\left(T^{*}\right), \bar{y}\left(T^{*}\right)\right]$ for all $t \in\left[0, T^{*}\right]$.

We claim that $\widetilde{u}$ is actually solution of $(\mathcal{P})$ in $C\left(\left[0, T^{*}\right], X\right)$. Indeed, from above, for all $t \in\left[0, T^{*}\right]$ we have $\widetilde{u}(t) \in[\underline{y}(t), \bar{y}(t)] \subset\left[\underline{y}\left(T^{*}\right), \bar{y}\left(T^{*}\right)\right]$ which in turn is include in $\left[\underline{y}\left(T^{\prime}\right), \bar{y}\left(T^{\prime}\right)\right]$. Therefore $\widetilde{F}(t, \widetilde{u}(t))=F(t, \widetilde{u}(t))$ so that $\widetilde{u}$ is solution of $(\mathcal{P})$. From now on we write $u$ for $\widetilde{u}$.

Step 2. We prove that there exists a global solution of $(\mathcal{P})$. We use the notation of Theorem 2.2 . and still denote by $u \in C\left(\left[0, T_{M a x}\right), X\right)$ the maximal solution of $(\mathcal{P})$.

By applying Theorem 2.2 it suffices to establish that there is no blow-up in finite time. Assume that $T_{\text {Max }}<+\infty$. From Step 1 we infer that for all $T<T_{\text {Max }}$, and all $t \in[0, T]$ we have $u(t) \in$ $\left[\underline{y}\left(T_{M a x}\right), \bar{y}\left(T_{M a x}\right)\right]$. Hence

$$
\|u\|_{C([0, T], X)} \leq \mathcal{L}_{N}(\Omega)^{\frac{1}{2}} \max \left(\left|\underline{y}\left(T_{M a x}\right)\right|,\left|\bar{y}\left(T_{M a x}\right)\right|\right),
$$

which makes $\lim _{T \rightarrow T_{\text {Max }}}\|u\|_{C([0, T], X)}=+\infty$ impossible.

Step 3. We finally establish that if $F$ is a regular CP-structured reaction functional, then $G$ : $[0,+\infty) \rightarrow L^{2}(\Omega)$, defined by $G(t)=F(t, u(t))$, belongs to $W^{1,1}\left(0, T, L^{2}(\Omega)\right)$ for all $T>0$. According to Theorem 2.1. we will infer that $u$ satisfies $\left(\mathrm{L}_{3}\right)$,

For all $s<t$ in $[0, T]$, and from the fact that $q, r$ and $u$ are absolutely continuous, we have

$$
\begin{aligned}
&\|F(t, u(t))-F(s, u(s))\|_{X} \leq\|F(t, u(t))-F(s, u(t))\|_{X}+\|F(s, u(t))-F(s, u(s))\|_{X} \\
& \leq\|q(t)-q(s)\|_{X}+\sup _{\zeta \in[\underline{y}(T), \bar{y}(T)]}|g(\zeta)|\|r(t)-r(s)\|_{L^{2}\left(\Omega, \mathbb{R}^{l}\right)} \\
& \quad+\|r\|_{L^{\infty}\left([0, T] \times \mathbb{R}^{N}, \mathbb{R}^{l}\right)} L_{g}\|u(t)-u(s)\|_{X}
\end{aligned}
$$

where ${ }^{4}$ the function $\varphi_{u}:[0, T] \rightarrow \mathbb{R}_{+}$, given by

$$
\varphi_{u}(\tau)=\left\|\frac{d q}{d t}(\tau)\right\|_{X}+\sup _{\zeta \in[\underline{y}(T), \bar{y}(T)]}|g(\zeta)|\left\|\frac{d r}{d t}(\tau)\right\|_{L^{2}\left(\Omega, \mathbb{R}^{l}\right)}+\|r\|_{L^{\infty}\left([0, T] \times \mathbb{R}^{N}, \mathbb{R}^{l}\right)} L_{g}\left\|\frac{d u}{d t}(\tau)\right\|
$$

belongs to $L^{1}(0, T)$. From (7), we easily deduce that $G$ is absolutely continuous, then belongs to $W^{1,1}(0, T, X)$. This completes the proof.

By an easy adaptation of the previous proof, applying this time Proposition 3.2 , we obtain the following result.

Theorem 3.2. Let $F$ be a CP-structured reaction functional, with $\underline{\rho}, \bar{\rho}$ and $\underline{y}, \bar{y}$ given by $(\mathrm{CP})$, and let $\Phi$ be the functional of the calculus of variations (5). Assume that $\underline{\underline{\rho}} \leq 0 \leq \bar{\rho}$. Then for any $T>0$, the Cauchy problem

$$
(\mathcal{P})\left\{\begin{array}{l}
\frac{d u}{d t}(t)+D \Phi(u(t))=F(t, u(t)) \text { for a.e. } t \in(0, T) \\
u(0)=u_{0}, \quad \underline{\rho} \leq u_{0} \leq \bar{\rho}, \quad u_{0} \in \overline{\operatorname{dom}(D \Phi)}
\end{array}\right.
$$

admits a unique solution $u \in C\left([0, T], L^{2}(\Omega)\right)$ satisfying assertions $\left(\mathrm{L}_{1}\right),\left(\mathrm{L}_{2}\right)$ and the following bounds in $[0, T]: \underline{y}(T) \leq \underline{y}(t) \leq u(t) \leq \bar{y}(t) \leq \bar{y}(T)$. If furthermore $F$ is a regular CP-structured reaction functional, then u satisfies $\left(\mathrm{L}_{3}\right)$,

Remark 3.3. 1) The set of functions $u_{0} \in \overline{\operatorname{dom}(D \Phi)}$ satisfying $\rho \leq u_{0} \leq \bar{\rho}$ is non empty. For the functional 44 of Theorem 3.1. any constant in $[\underline{\rho}, \bar{\rho}]$ is suitable since $H^{1}(\Omega)=\operatorname{dom}(\Phi) \subset$ $\overline{\operatorname{dom}(\Phi)}=\overline{\operatorname{dom}(D \Phi)}$ (for the last equality we refer to [, Lemma 17.4.1]). For the functional (5) of Theorem 3.2, $u_{0}=0$ is suitable.

\footnotetext{
${ }^{4}$ We still wrote $L g$ the Lipchitz constant of the restriction of $g$ to $[\underline{y}(T), \bar{y}(T)]$.
} 
2) In the proof of Theorem 3.1, we have established that if $u$ is the solution of $(\mathcal{P})$, then, for all $t \in[0, T]$, the function $F(t, u(t))$ belongs to $L^{2}(\Omega)$ since $F(t, u(t))=\widetilde{F}(t, \widetilde{u}(t))$ for all $t \in[0, T]$.

3) In Theorem 3.1. the mixed Dirichlet-Neumann boundary condition fulfilled by the solution $u$ at $t \in] 0, T]$, is expressed in condition $\left(\mathrm{L}_{3}\right)$ by the fact that $u(t) \in \operatorname{dom}(D \Phi)$ for all $\left.\left.t \in\right] 0, T\right]$, and is given by:

$$
a_{0} u(t)+D_{\xi} W(\cdot, \nabla u(t)) \cdot \mathbf{n}=h \text { on } \partial \Omega .
$$

Therefore, when $F$ is a regular CP-structured reaction functional, $(\mathcal{P})$ may be written as

$$
(\mathcal{P})\left\{\begin{array}{l}
\left.\left.\frac{d u}{d t}(t)-\operatorname{div} D_{\xi} W(\cdot, \nabla u(t))=F(t, u(t)) \text { for all } t \in\right] 0, T\right] \quad\left(\text { equality in } L^{2}(\Omega)\right) \\
u(0)=u_{0}, \quad \underline{\rho} \leq u_{0} \leq \bar{\rho}, \quad u_{0} \in \overline{\operatorname{dom}(D \Phi)} \\
\left.\left.a_{0} u(t)+D_{\xi} W(\cdot, \nabla u(t)) \cdot \mathbf{n}=h \text { on } \partial \Omega \text { for all } t \in\right] 0, T\right] .
\end{array}\right.
$$

4) As regards Theorem 3.2 , the same remark holds, i.e., problem $(\mathcal{P})$ may be written as

$$
(\mathcal{P})\left\{\begin{array}{l}
\left.\left.\frac{d u}{d t}(t)-\operatorname{div} D_{\xi} W(\cdot, \nabla u(t))=F(t, u(t)) \text { for all } t \in\right] 0, T\right] \quad\left(\text { equality in } L^{2}(\Omega)\right) \\
u(0)=u_{0}, \quad \underline{\rho} \leq u_{0} \leq \bar{\rho}, \quad u_{0} \in \overline{\operatorname{dom}(D \Phi)}, \\
u(t)=0 \text { on } \Gamma \text { for all } t \in] 0, T], \\
\left.\left.D_{\xi} W(\cdot, \nabla u(t)) \cdot \mathbf{n}=0 \text { on } \partial \Omega \backslash \Gamma \text { for all } t \in\right] 0, T\right] .
\end{array}\right.
$$

3.5. Estimate of the $L^{2}(\Omega)$-norm of the right derivative. From above we know that when the CPstructured reaction functional is regular, the solution of $\mathcal{P}$ admits a right derivative at each $t \in(0, T]$. The next estimate below is crucial in the proof of the compactness step (Step 2) of the convergence theorem, Theorem 4.1 .

Proposition 3.3. Under hypotheses of Theorems 3.1, 3.2, when $F$ is a regular CP-structured reaction functional, for all $t \in(0, T]$ we have

$$
\left\|\frac{d u^{+}}{d t}(t)\right\|_{X} \leq C+\left(C+\frac{1}{t}\right) \int_{0}^{t}\left\|\frac{d u}{d t}(\tau)\right\|_{X} d \tau
$$

where

$$
C=\max \left(L_{g}\|r\|_{L^{\infty}\left([0, T] \times \mathbb{R}^{N}, \mathbb{R}^{l}\right)}, \int_{0}^{T}\left\|\frac{d q}{d t}(\tau)\right\|_{X} d \tau+\sup _{\zeta \in[\underline{y}(T), \bar{y}(T)]}|g(\zeta)| \int_{0}^{T}\left\|\frac{d r}{d t}(\tau)\right\|_{L^{2}\left(\Omega, \mathbb{R}^{l}\right)} d \tau\right),
$$

and $L g$ denotes the Lipschitz constant of the restriction of $g$ to $[\underline{\rho}, \bar{\rho}]$.

Proof. Step 1. We establish the following lemma.

Lemma 3.3. Let $X$ be a Hilbert space, $T>0, G \in W^{1,1}(0, T, X)$ and $\Phi: X \rightarrow \mathbb{R} \cup\{+\infty\}$ be a convex proper lower semicontinuous functional. Let $u$ satisfy

$$
\left\{\begin{array}{l}
\frac{d u}{d t}(t)+\partial \Phi(u(t)) \ni G(t) \text { for a.e. } t \in(0, T), \\
u(0) \in \overline{\operatorname{dom}(\partial \Phi)} .
\end{array}\right.
$$

Then the right derivative of $u$ satisfies for all $t \in] 0, T]$ the following estimate

$$
\left\|\frac{d u^{+}}{d t}(t)\right\|_{X} \leq \frac{1}{t} \int_{0}^{t}\left\|\frac{d u}{d t}(s)\right\|_{X} d s+\int_{0}^{t}\left\|\frac{d G}{d t}(s)\right\|_{X} d s .
$$

Proof. For $h>0$ intended to tend to 0 , set $H:=G(\cdot+h)$ and let $v$ be the solution of

$$
\left\{\begin{array}{l}
\frac{d v}{d t}(t)+\partial \Phi(v(t)) \ni H(t) \text { for a.e. } t \in(0, T), \\
v(0)=u(h) .
\end{array}\right.
$$


Clearly $v(t)=u(t+h)$ (recall that $u$ which solves $(10)$ is the restriction to $[0, T]$ of a unique global solution $u \in C([0,+\infty), X)$ of $(10)$ in $(0,+\infty)$, and that $u(t) \in \operatorname{dom}(\Phi)$ for all $t \in(0, T))$. From (10), (11), and the monotonicity of $\partial \Phi$, we infer that for a.e. $\sigma \in(0, T)$

$$
\left\langle\frac{d v}{d t}(\sigma)-\frac{d u}{d t}(\sigma), v(\sigma)-u(\sigma)\right\rangle \leq\langle H(\sigma)-G(\sigma), v(\sigma)-u(\sigma)\rangle,
$$

hence

$$
\frac{1}{2} \frac{d}{d t}\|v(\sigma)-u(\sigma)\|_{X}^{2} \leq\langle H(\sigma)-G(\sigma), v(\sigma)-u(\sigma)\rangle .
$$

Integrating over $(s, t)$ where $0 \leq s \leq t \leq T$, we obtain for all $t \in[0, T]$

$$
\begin{aligned}
\frac{1}{2}\|v(t)-u(t)\|_{X}^{2} & \leq \frac{1}{2}\|v(s)-u(s)\|_{X}^{2}+\int_{s}^{t}\langle H(\sigma)-G(\sigma), v(\sigma)-u(\sigma)\rangle d \sigma \\
& \leq \frac{1}{2}\|v(s)-u(s)\|_{X}^{2}+\int_{0}^{t} \mid H(\sigma)-G(\sigma)\left\|_{X}\right\| v(\sigma)-u(\sigma) \| d \sigma .
\end{aligned}
$$

Thus, according to the Gronvall type lemma, [13, Lemma A.5], it follows that for all $t \in[0, T]$ and all $s \in[0, t]$

that is

$$
\|v(t)-u(t)\|_{X} \leq\|v(s)-u(s)\|_{X}+\int_{0}^{t} \mid H(\sigma)-G(\sigma) \|_{X} d \sigma
$$

$$
\|u(t+h)-u(t)\|_{X} \leq\|u(s+h)-u(s)\|_{X}+\int_{0}^{t} \mid G(\sigma+h)-G(\sigma) \|_{X} d \sigma .
$$

Dividing by $h$ and letting $h \rightarrow 0$, we infer that for all $t \in[0, T]$ and all $s \in[0, t]$

$$
\left\|\frac{d u^{+}}{d t}(t)\right\|_{X} \leq\left\|\frac{d u^{+}}{d t}(s)\right\|_{X}+\int_{0}^{t}\left\|\frac{d G}{d t}(\sigma)\right\|_{X} d \sigma
$$

(for a justification in order to obtain the last integral, we refer the reader to [13, Proposition A2]). By integration over $(0, t)$, we obtain for all $t \in[0, T]$

$$
\begin{aligned}
t\left\|\frac{d u^{+}}{d t}(t)\right\|_{X} & \leq \int_{0}^{t}\left\|\frac{d u^{+}}{d t}(s)\right\|_{X}+t \int_{0}^{t}\left\|\frac{d G}{d t}(s)\right\|_{X} d s \\
& =\int_{0}^{t}\left\|\frac{d u}{d t}(s)\right\|_{X}+t \int_{0}^{t}\left\|\frac{d G}{d t}(s)\right\|_{X} d s
\end{aligned}
$$

which gives the result for all $t \in] 0, T]$. This ends the proof of Lemma 3.3

Last step. The thesis of Proposition 3.3 follows by combining Lemma 3.3 and the expression of the total variation of $G$ given by (7) and (8) where $G(t)=F(t, u(t))$.

\section{General Convergence theorem for a Class of nonlinear ReaCtion-Diffusion problems}

Consider a sequence $\left(\Phi_{n}\right)_{n \in \mathbb{N}}$ of functionals of the calculus of variations $\Phi_{n}: L^{2}(\Omega) \rightarrow \mathbb{R} \cup\{+\infty\}$ defined by

$$
\Phi_{n}(u)=\left\{\begin{array}{cl}
\int_{\Omega} W_{n}(x, \nabla u(x)) d x+\frac{1}{2} \int_{\partial \Omega} a_{0, n} u^{2} d \mathcal{H}_{N-1}-\int_{\partial \Omega} h_{n} u d \mathcal{H}_{N-1} & \text { if } u \in H^{1}(\Omega) \\
+\infty & \text { otherwise }
\end{array}\right.
$$

where $h_{n} \in L_{\mathcal{H}_{N-1}}^{2}(\partial \Omega), a_{0, n} \in L_{\mathcal{H}_{N-1}}^{\infty}(\partial \Omega), a_{0, n} \geq 0 \mathcal{H}_{N-1}$-a.e. in $\partial \Omega, a_{0, n} \geq \sigma_{n}$ on $\Gamma \subset \partial \Omega$ with $\mathcal{H}_{N-1}(\Gamma)>0$ for some $\sigma_{n}>0$, and $W_{n}: \mathbb{R}^{N} \times \mathbb{R}^{N} \rightarrow \mathbb{R}$ is a measurable function satisfying the following conditions:

$\left(\mathrm{D}_{1, n}\right)$ there exist $\left\{\alpha_{n}\right\}_{n \in \mathbb{N}} \subset \mathbb{R}_{+}^{*}$ and $\left\{\beta_{n}\right\}_{n \in \mathbb{N}} \subset \mathbb{R}_{+}^{*}$ such that for a.e. $x \in \mathbb{R}^{N}$, all $\xi \in \mathbb{R}^{N}$, and all $n \in \mathbb{N}$

$$
\alpha_{n}|\xi|^{2} \leq W_{n}(x, \xi) \leq \beta_{n}\left(1+|\xi|^{2}\right),
$$

$\left(\mathrm{D}_{2, n}\right)$ for a.e. $x \in \mathbb{R}^{N}$ and every $n \in \mathbb{N}$, the function $W_{n}(x, \cdot)$ is convex and differentiable with $D_{\xi} W_{n}(x, 0)=0$ a.e. in $\mathbb{R}^{N}$, 
$\left(\mathrm{D}_{3, n}\right)\left(W_{n}\right)_{n \in \mathbb{N}}$ is uniformly strongly convex, i.e., for some $\gamma>0$ it holds for all $\xi \in \mathbb{R}^{N}$,

$$
\inf _{n \in \mathbb{N}} \inf _{x \in \mathbb{R}^{N}} D_{\xi} W_{n}(x, \xi) \cdot \xi \geq \gamma|\xi|^{2} .
$$

In the following, we fix $T>0$ and we consider a sequence $\left(F_{n}\right)_{n \in \mathbb{N}}$ of regular CP-structured reaction functionals, each of them being associated with $\left(r_{n}, g_{n}, q_{n}\right)$, i.e., $F_{n}(t, v)(x)=f_{n}(t, x, v(x))$ for all $t \in[0, T]$, a.e. $x \in \Omega$ and all $v \in L^{2}(\Omega)$, where

$$
f_{n}(t, x, \zeta)=r_{n}(t, x) \cdot g_{n}(\zeta)+q_{n}(t, x) \text { for all }(t, x, \zeta) \in[0,+\infty) \times \mathbb{R}^{N} \times \mathbb{R} .
$$

We assume that for all $n \in \mathbb{N}$ the fonction $g_{n}$ is locally Lipschitz continuous, uniformly with respect to $n$, i.e., for every interval $I \subset \mathbb{R}$ there exists $L_{I} \geq 0$ such that for every $\left(\zeta, \zeta^{\prime}\right) \in \mathbb{R}^{2}$

$$
\sup _{n \in \mathbb{N}}\left|g_{n}(\zeta)-g_{n}\left(\zeta^{\prime}\right)\right| \leq L_{I}\left|\zeta-\zeta^{\prime}\right|
$$

This condition is fulfilled for example by $g_{n}=\left(g_{n, i}\right)_{i=1, \ldots, l}$ where the scalar functions $g_{n, i}$ are convex and satisfy for all $\zeta \in \mathbb{R}, 0 \leq g_{n, i}(\zeta) \leq \beta_{i}\left(1+|\zeta|^{p_{i}}\right)$ for some $\beta_{i}>0$ and $p_{i} \geq 1$. This is the case of Example A.1 $b$ ) where $\gamma_{n}>0$ is substitute for $\gamma>0$.

We assume that the absolute continuity of the functions $r_{n}$ and $q_{n}$ holds uniformly with respect to $n$, i.e.,

$$
\begin{aligned}
& \sup _{n \in \mathbb{N}} \int_{0}^{T}\left\|\frac{d r_{n}}{d t}(t, \cdot)\right\|_{L^{2}\left(\Omega, \mathbb{R}^{l}\right)} d t<+\infty, \\
& \sup _{n \in \mathbb{N}} \int_{0}^{T}\left\|\frac{d q_{n}}{d t}(t, \cdot)\right\|_{L^{2}(\Omega)} d t<+\infty .
\end{aligned}
$$

Finally, we assume that

$$
\underline{\rho}:=\inf _{n \in \mathbb{N}} \underline{y}_{n}(T)>-\infty \quad \text { and } \quad \bar{\rho}:=\sup _{n \in \mathbb{N}} \bar{y}_{n}(T)<+\infty,
$$

and, for all $n \in \mathbb{N}$,

$$
a_{0, n} \underline{\rho}_{n} \leq h_{n} \leq a_{0, n} \bar{\rho}_{n} \text { on } \partial \Omega
$$

where $\underline{y}_{n}$ and $\bar{y}_{n}$ are given by condition $(\mathrm{CP})$ fulfilled by each $F_{n}$. Recall that these two functions are solution of suitable o.d.e. with initial condition $\underline{\rho}_{n}$ and $\bar{\rho}_{n}$ respectively. When considering the case

$$
a_{0, n}(x)= \begin{cases}0 & \text { if } x \in \partial \Omega \backslash \Gamma \\ +\infty & \text { if } x \in \Gamma\end{cases}
$$

and $h_{n}=0$, for all $n \in \mathbb{N}$, then 16 has to be replaced by

$$
\underline{\rho}_{n} \leq 0 \leq \bar{\rho}_{n} \quad \text { for all } n \in \mathbb{N} .
$$

In order to establish a convergence result for reaction-diffusion problems $\left(\mathcal{P}_{n}\right)$ with diffusion part $D \Phi_{n}$ and reaction part $F_{n}$, we take advantage of standard results involving $\Gamma$-convergence of the functionals $\Phi_{n}$ to $\Phi$, and particularly in homogenization framework (see [8, Subsection 12.4]). More precisely, it is convenient to establish the convergence of the sequence of problems $\left(\mathcal{P}_{n}\right)$ under the hypothesis of the Mosco-convergence of the sequence $\left(\Phi_{n}\right)_{n \in \mathbb{N}}$, introduced in [18, 19], i.e., the $\Gamma$-convergence of the functionals $\Phi_{n}$ when $L^{2}(\Omega)$ is equipped both with its strong and its weak topology. For the definition and variational properties of this notion we refer the reader to [8, Section 17.4.2], and for the connection with Moreau-Yosida approximations we refer to [7, 16. Note that even if $\Phi_{n}$ is Gâteaux differentiable according to $\left(\mathrm{D}_{2, n}\right)$ we are not ensured that its Mosco-limit $\Phi$ is Gâteaux differentiable. We will denote by $\Phi_{n} \stackrel{M}{\rightarrow} \Phi$ the Mosco-convergence of the sequence $\left(\Phi_{n}\right)_{n \in \mathbb{N}}$ to $\Phi$. A first important lemma (which is proved in Appendix C concerns the Mosco-convergence of functionals defined in $L^{2}(0, T, X)$.

Lemma 4.1. Let $\left(X,\|\cdot\|_{X}\right)$ be a reflexive Banach space whose norm together with its dual norm is strictly convex, and such that weak convergence of sequences and convergence of their norms imply strong convergence. Let $\left(\psi_{n}\right)_{n \in \mathbb{N}}, \psi$ be a sequence of convex uniformly proper lower semicontinuous functions 
from $X$ into $\mathbb{R} \cup\{+\infty\}$ such that $\psi_{n} \stackrel{M}{\rightarrow} \psi$ and consider $\left(\Psi_{n}\right)_{n \in \mathbb{N}}, \Psi: L^{2}(0, T, X) \rightarrow \mathbb{R} \cup\{+\infty\}$ defined by

Then $\Psi_{n} \stackrel{M}{\rightarrow} \Psi$.

$$
\Psi_{n}(v):=\int_{0}^{T} \psi_{n}(v(t)) d t ; \Psi(v):=\int_{0}^{T} \psi(v(t)) d t .
$$

Recall that the sequence $\left(\psi_{n}\right)_{n \in \mathbb{N}}, \psi$ is said to be uniformly proper if $\psi$ is proper and if there exists a bounded sequence $\left(u_{n, 0}\right)_{n \in \mathbb{N}}$ in $X$ such that $\sup _{n \in \mathbb{N}} \psi_{n}\left(u_{n, 0}\right)<+\infty$.

Here is the main result of Section 4 .

Theorem 4.1 (General convergence theorem). Assume that $\left(W_{n}\right)_{n \in \mathbb{N}}$ satisfies $\left(\mathrm{D}_{1, n}\right)$, $\left(\mathrm{D}_{2, n}\right)$, and $\left(\mathrm{D}_{3, n}\right)$, and that the sequence of CP-structured reaction functionals $\left(F_{n}\right)_{n \in \mathbb{N}}$, of the form (12), satisfies (13), 14), 15], 116) or (17) when, for all $n \in \mathbb{N}, h_{n}=0$ and

$$
a_{0, n}(x)= \begin{cases}0 & \text { if } x \in \partial \Omega \backslash \Gamma \\ +\infty & \text { if } x \in \Gamma .\end{cases}
$$

Let $u_{n}$ be the unique solution of the Cauchy problem

$$
\left(\mathcal{P}_{n}\right)\left\{\begin{array}{l}
\frac{d u_{n}}{d t}(t)+D \Phi_{n}\left(u_{n}(t)\right)=F_{n}\left(t, u_{n}(t)\right) \text { for a.e. } t \in(0, T) \\
u_{n}(0)=u_{n}^{0}, \quad \underline{\rho}_{n} \leq u_{n}^{0} \leq \bar{\rho}_{n}, \quad u_{n}^{0} \in \operatorname{dom}\left(\Phi_{n}\right) .
\end{array}\right.
$$

Assume that

$\left(\mathrm{H}_{1}\right) \Phi_{n} \stackrel{M}{\rightarrow} \Phi$ and $\sup _{n \in \mathbb{N}}\left\|h_{n}\right\|_{L_{\mathcal{H}_{N-1}}^{2}(\partial \Omega)}<+\infty ;$

$\left(\mathrm{H}_{2}\right) \sup _{n \in \mathbb{N}} \Phi_{n}\left(u_{n}^{0}\right)<+\infty$;

$\left(\mathrm{H}_{3}\right)$ there exists $u^{0}$ such that $u_{n}^{0} \rightarrow u^{0}$ strongly in $L^{2}(\Omega)$;

$\left(\mathrm{H}_{4}\right)$ there exists $g$ such that $g_{n}$ pointwise converge to $g$;

$\left(\mathrm{H}_{5}\right) \sup _{n \in \mathbb{N}}\left\|r_{n}\right\|_{L^{\infty}\left([0, T] \times \mathbb{R}^{N}, \mathbb{R}^{l}\right)}<+\infty$, and there exists $r \in L^{\infty}\left([0, T] \times \mathbb{R}^{N}, \mathbb{R}^{l}\right)$

such that $r_{n} \rightarrow r$ in $L^{2}\left(0, T, L^{2}\left(\Omega, \mathbb{R}^{l}\right)\right)$;

$\left(\mathrm{H}_{6}\right)$ for all $t \in[0, T], \sup _{n \in \mathbb{N}}\left\|q_{n}(t, \cdot)\right\|_{L^{2}(\Omega)}<+\infty$, and there exists $q$ such that $q_{n} \rightarrow q$ in $L^{2}\left(0, T, L^{2}(\Omega)\right)$.

Then $\left(u_{n}\right)_{n \in \mathbb{N}}$ uniformly converges in $C\left([0, T], L^{2}(\Omega)\right)$ to the unique solution of the problem

$$
(\mathcal{P})\left\{\begin{array}{l}
\frac{d u}{d t}(t)+\partial \Phi(u(t)) \ni F(t, u(t)) \text { for a.e. } t \in(0, T) \\
u(0)=u^{0}, \quad \underline{\rho}:=\inf _{n \in \mathbb{N}} \underline{y}_{n}(T) \leq u^{0} \leq \sup _{n \in \mathbb{N}} \bar{y}_{n}(T):=\bar{\rho}, \quad u_{0} \in \operatorname{dom}(\Phi) .
\end{array}\right.
$$

The reaction functional $F:[0,+\infty) \times L^{2}(\Omega) \rightarrow \mathbb{R}^{\Omega}$ is defined, for all $t \in[0, T]$, all $v \in L^{2}(\Omega)$ and for a.e. $x \in \Omega$, by

$$
F(t, v)(x)=f(t, x, v(x)) \quad \text { and } \quad f(t, x, \zeta)=r(t, x) \cdot g(\zeta)+q(t, x) .
$$

Moreover, $\frac{d u_{n}}{d t} \rightarrow \frac{d u}{d t}$ weakly in $L^{2}\left(0, T, L^{2}(\Omega)\right)$ and $\inf _{n \in \mathbb{N}} \underline{y}_{n}(T) \leq u \leq \sup _{n \in \mathbb{N}} \bar{y}_{n}(T)$.

If furthermore $\Phi\left(u_{n}^{0}\right) \rightarrow \Phi\left(u^{0}\right), r_{n} \rightarrow r$ in $L^{2}\left(0, T, L^{2}\left(\Omega, \mathbb{R}^{l}\right)\right)$, and $q_{n} \rightarrow q$ in $L^{2}\left(0, T, L^{2}(\Omega)\right)$, then $\frac{d u_{n}}{d t} \rightarrow \frac{d u}{d t}$ in $L^{2}\left(0, T, L^{2}(\Omega)\right)$.

Proof. We only establish the proof for $\Phi_{n}$ given of the first form, i.e., when the domain of the subdifferntial contains mixed Dirichlet-Neumann boundary conditions. The proof of the second case is slightly shorter, with some easy adaptations. Note that in the statement of Theorem 4.1, we assume that $u_{n}^{0} \in H^{1}(\Omega)=\operatorname{dom}\left(\Phi_{n}\right)$. But $\operatorname{dom}\left(\Phi_{n}\right) \subset \overline{\operatorname{dom}\left(\Phi_{n}\right)}=\overline{\operatorname{dom}\left(D \Phi_{n}\right)}$, thus $u_{n}^{0} \in \overline{\operatorname{dom}\left(D \Phi_{n}\right)}$. Therefore, according to Theorem 3.1. $\left(\mathcal{P}_{n}\right)$ has a unique solution $u_{n}$ which satisfies $\left(\mathrm{L}_{2}\right)$ and $\left(\mathrm{L}_{3}\right)$ of Theorem 2.1 . 
and the bounds $\underline{y}_{n}(T) \leq u_{n} \leq \bar{y}_{n}(T)$. Finally, note that since $u_{n}^{0} \in \operatorname{dom}\left(\Phi_{n}\right)$, we have the additional regularity: $\frac{d u_{n}}{d t}$ belongs to $L^{2}\left(0, T, L^{2}(\Omega)\right)$ (see [8, Theorem 17.2.5] or [13, Theorem 3.6]).

Step 1. We establish

$$
\begin{aligned}
& \sup _{n \in \mathbb{N}}\left\|u_{n}\right\|_{C(0, T, X)} \leq \mathcal{L}_{N}(\Omega)^{\frac{1}{2}} \max (|\underline{\rho}|,|\bar{\rho}|) ; \\
& \sup _{n \in \mathbb{N}}\left\|\frac{d u_{n}}{d t}\right\|_{L^{2}(0, T, X)}<+\infty
\end{aligned}
$$

(recall that $\rho$ and $\bar{\rho}$ belong to $\mathbb{R}$ from $(15 p)$.

Inequality (18) follows directly from $\underline{\rho} \leq \underline{y}_{n}(T) \leq u_{n} \leq \bar{y}_{n}(T) \leq \bar{\rho}$. Let us establish (19). In what follows the letter $C$ denotes a constant which can vary from line to line. From $\left(\mathcal{P}_{n}\right)$ we deduce that for a.e. $t \in(0, T)$,

$$
\left\|\frac{d u_{n}}{d t}(t)\right\|_{X}^{2}+\left\langle D \Phi_{n}\left(u_{n}(t)\right), \frac{d u_{n}}{d t}(t)\right\rangle=\left\langle F_{n}\left(t, u_{n}(t)\right), \frac{d u_{n}}{d t}(t)\right\rangle .
$$

We have used the fact that $F_{n}\left(t, u_{n}(t)\right)$ belongs to $L^{2}(\Omega)$ as stated in Remark 3.3 . By integrating this equality over $(0, T)$, we obtain

$$
\int_{0}^{T}\left\|\frac{d u_{n}}{d t}(t)\right\|_{X}^{2} d t+\int_{0}^{T}\left\langle D \Phi_{n}\left(u_{n}(t)\right), \frac{d u_{n}}{d t}(t)\right\rangle d t=\int_{0}^{T}\left\langle F_{n}\left(t, u_{n}(t)\right), \frac{d u_{n}}{d t}(t)\right\rangle d t .
$$

But $\frac{d u_{n}}{d t}$ belongs to $L^{2}(0, T, X)$ and $t \mapsto \Phi_{n}\left(u_{n}(t)\right)$ is absolutely continuous (see [13, Theorem 3.6]). Consequently for a.e. $t \in(0, T), \frac{d}{d t} \Phi\left(u_{n}(t)\right)=\left\langle D \Phi u_{n}(t), \frac{d u_{n}}{d t}(t)\right\rangle$ (see [8, Proposition 17.2.5]). Recall that there exists $\mu>0$ such that $\Phi_{n}+\mu\left(\|\cdot\|_{X}+1\right) \geq 0$. Therefore from (20) and (18) we deduce

$$
\begin{aligned}
\int_{0}^{T}\left\|\frac{d u_{n}}{d t}(t)\right\|_{X}^{2} d t & =-\Phi_{n}\left(u_{n}(T)\right)+\Phi_{n}\left(u_{n}^{0}\right)+\int_{0}^{T}\left\langle F_{n}\left(t, u_{n}(t)\right), \frac{d u_{n}}{d t}(t)\right\rangle d t \\
& \leq \mu\left(\left\|u_{n}(T)\right\|_{X}+1\right)+\sup _{n \in \mathbb{N}} \Phi_{n}\left(u_{n}^{0}\right) \\
& +\left(\int_{0}^{T}\left\|F_{n}\left(t, u_{n}(t)\right)\right\|_{X}^{2} d t\right)^{\frac{1}{2}}\left(\int_{0}^{T}\left\|\frac{d u_{n}}{d t}(t)\right\|_{X}^{2} d t\right)^{\frac{1}{2}} \\
& \leq C+\sup _{n \in \mathbb{N}} \Phi_{n}\left(u_{n}^{0}\right)+\left(\int_{0}^{T}\left\|F_{n}\left(t, u_{n}(t)\right)\right\|_{X}^{2} d t\right)^{\frac{1}{2}}\left(\int_{0}^{T}\left\|\frac{d u_{n}}{d t}(t)\right\|_{X}^{2} d t\right)^{\frac{1}{2}} .
\end{aligned}
$$

By using the structure of the CP-structured reaction functional $F_{n}$, we have

$$
\left\|F_{n}\left(t, u_{n}(t)\right)\right\|_{X}^{2}=\left\|\widetilde{F}_{n}\left(t, u_{n}(t)\right)\right\|_{X}^{2} \leq 2\left\|F_{n}(t, 0)\right\|_{X}^{2}+2 L^{2}\left\|u_{n}(t)\right\|_{C([0, T], X)}^{2}
$$

where $L=L_{I} \sup _{n \in \mathbb{N}}\left\|r_{n}\right\|_{L^{\infty}\left([0, T] \times \mathbb{R}^{N}, \mathbb{R}^{l}\right)}$, and $L_{I}$ is the Lipschitz constant of $\left\{g_{n}\right\}_{n \in \mathbb{N}}$ in the interval $I=[\underline{\rho}, \bar{\rho}]$. On the other hand, we have clearly

$$
\left\|F_{n}(t, 0)\right\|_{X}^{2} \leq C\left(1+\left\|q_{n}(t, \cdot)\right\|_{X}^{2}\right)
$$

where, from hypothesis $\left(\mathrm{H}_{4}\right)$ and $\left(\mathrm{H}_{5}\right), C$ is a nonnegative constant which does not depend on $n$. Hence

$$
\int_{0}^{T}\left\|F_{n}(t, 0)\right\|_{X}^{2} d t \leq C\left(1+\left\|q_{n}\right\|_{L^{2}(0, T, X)}^{2}\right)
$$

so that, according to hypothesis $\left(\mathrm{H}_{6}\right)$

$$
\sup _{n \in \mathbb{N}} \int_{0}^{T}\left\|F_{n}(t, 0)\right\|_{X}^{2} d t<+\infty .
$$

Combining (18) and 25), 23) yields

$$
\sup _{n \in \mathbb{N}} \int_{0}^{T}\left\|F_{n}\left(t, u_{n}(t)\right)\right\|_{X}^{2} d t<+\infty .
$$


From 22 and $\left(\mathrm{H}_{2}\right)$, we infer that

$$
\int_{0}^{T}\left\|\frac{d u_{n}}{d t}(t)\right\|_{X}^{2} d t \leq C\left(1+\left(\int_{0}^{T}\left\|\frac{d u_{n}}{d t}(t)\right\|_{X}^{2} d t\right)^{\frac{1}{2}}\right)
$$

where $C$ is a nonnegative constant which does not depend on $n$, from which we deduce (19).

Step 2. We prove that there exist $u \in C([0, T], X)$, and a subsequence of $\left(u_{n}\right)_{n \in \mathbb{N}}$ not relabeled, satisfying $u_{n} \rightarrow u$ in $C([0, T], X)$.

We apply the Ascoli-Arzela compactness theorem. From $\left[18,\left(u_{n}\right)_{n \in \mathbb{N}}\right.$ is bounded in $C([0, T], X)$. Moreover, for $s<t,(s, t) \in\left[0, T^{2}\right]$, we have

$$
\left\|u_{n}(t)-u_{n}(s)\right\|_{X} \leq \int_{s}^{t}\left\|\frac{d u_{n}}{d t}(\tau)\right\|_{X} d \tau \leq(t-s)^{\frac{1}{2}}\left\|\frac{d u_{n}}{d t}\right\|_{L^{2}(0, T, X)} \leq(t-s)^{\frac{1}{2}} \sup _{n \in \mathbb{N}}\left\|\frac{d u_{n}}{d t}\right\|_{L^{2}(0, T, X)}
$$

which, according to 19 , proves the equicontinuity of the sequence $\left(u_{n}\right)_{n \in \mathbb{N}}$. It remains to establish for each $t \in[0, T]$, the relative compactness in $X$ of the set $E_{t}:=\left\{u_{n}(t): n \in \mathbb{N}\right\}$. For $t=0$ there is nothing to prove because of hypothesis $\left(\mathrm{H}_{3}\right)$ on the initial condition. For $\left.\left.t \in\right] 0, T\right]$ we are going to use that $H^{1}(\Omega)$ is compactly embedded in $L^{2}(\Omega)$. For that purpose, we are going to see that the boundedness of $\nabla u_{n}(t)$ requires the sharp estimate of Proposition 3.3 .

According to Theorem 3.1, $u_{n}$ satisfies $\left(\mathrm{L}_{3}\right)$, then possesses a right derivative at each $\left.\left.t \in\right] 0, T\right]$ (at $t=T$, this is due to the fact that $u_{n}$ belongs to $C([0,+\infty), X)$ so that the right derivative of $u_{n}$ at $t=T$ is nothing but the right derivative of the restriction of $u_{n}$ to $\left.[0, T]\right)$. Moreover,

$$
\left.\left.\frac{d u_{n}^{+}}{d t}(t)+D \Phi_{n}\left(u_{n}(t)\right)=F_{n}\left(t, u_{n}(t)\right) \text { for all } t \in\right] 0, T\right]
$$

Taking $u_{n}(t)$ as a test function, we infer that for all $\left.\left.t \in\right] 0, T\right]$

$$
\left\langle\frac{d u_{n}^{+}}{d t}(t), u_{n}(t)\right\rangle+\left\langle D \Phi_{n}\left(u_{n}(t)\right), u_{n}(t)\right\rangle=\left\langle F_{n}\left(t, u_{n}(t)\right), u_{n}(t)\right\rangle
$$

hence, from the Green formula and the fact that $u_{n}(t) \in \operatorname{dom} D \Phi_{n}$ for all $\left.\left.t \in\right] 0, T\right]$,

$$
\begin{aligned}
& \int_{\Omega} D_{\xi} W_{n}\left(x, \nabla u_{n}(t)\right) \cdot \nabla u_{n}(t) d x \\
& =\int_{\partial \Omega} D_{\xi} W_{n}\left(x, \nabla u_{n}(t)\right) \cdot \mathbf{n} u_{n}(t) d \mathcal{H}_{N-1}-\int_{\Omega} \frac{d u_{n}^{+}}{d t}(t) u_{n}(t) d x+\int_{\Omega} F_{n}\left(t, u_{n}(t)\right) u_{n}(t) d x \\
& =\int_{\partial \Omega}\left(\phi_{n}-a_{0, n} u_{n}(t)\right) u_{n}(t) d \mathcal{H}_{N-1}-\int_{\Omega} \frac{d u_{n}^{+}}{d t}(t) u_{n}(t) d x+\int_{\Omega} F_{n}\left(t, u_{n}(t)\right) u_{n}(t) d x \\
& \leq \int_{\partial \Omega} \phi_{n} u_{n}(t) d \mathcal{H}_{N-1}-\int_{\Omega} \frac{d u_{n}^{+}}{d t}(t) u_{n}(t) d x+\int_{\Omega} F_{n}\left(t, u_{n}(t)\right) u_{n}(t) d x .
\end{aligned}
$$

Choose $\nu \in \mathbb{R}, 0<\nu<\frac{2 \gamma}{C_{\text {trace }}}$, where $\gamma$ is the positive constant of the uniform strong convexity condition $\left(\mathrm{D}_{3, n}\right)$, and $C_{\text {trace }}$ is the constant of continuity of the trace operator. From $\left[26,,\left(\mathrm{D}_{3, n}\right)\right.$, and 18 we infer that for all $t \in] 0, T]$,

$$
\begin{aligned}
\gamma \int_{\Omega}\left|\nabla u_{n}(t)\right|^{2} d x & \leq\left\|\phi_{n}\right\|_{L_{\mathcal{H}_{N-1}}^{2}(\partial \Omega)}\left\|u_{n}(t)\right\|_{L_{\mathcal{H}_{N-1}}^{2}(\partial \Omega)} \\
& +\mathcal{L}_{N}(\Omega)^{\frac{1}{2}} \max (|\underline{\rho}|,|\bar{\rho}|)\left(\left\|\frac{d u_{n}^{+}}{d t}(t)\right\|_{X}+\left\|F_{n}\left(t, u_{n}(t)\right)\right\|_{X}\right) \\
& \leq \frac{C_{\text {trace }}}{2 \nu}\left\|\phi_{n}\right\|_{L_{\mathcal{H}_{N-1}}^{2}(\partial \Omega)}^{2}+\frac{C_{\text {trace }} \nu}{2}\left\|u_{n}(t)\right\|_{H^{1}(\Omega)}^{2} \\
& +\mathcal{L}_{N}(\Omega)^{\frac{1}{2}} \max (|\underline{\rho}|,|\bar{\rho}|)\left(\left\|\frac{d u_{n}^{+}}{d t}(t)\right\|_{X}+\left\|F_{n}\left(t, u_{n}(t)\right)\right\|_{X}\right) \\
& \leq \frac{C_{\text {trace }}}{2 \nu}\left\|\phi_{n}\right\|_{L_{\mathcal{H}_{N-1}}^{2}}^{2}(\partial \Omega)+\frac{C_{\text {trace }} \nu}{2}\left(\int_{\Omega}\left|\nabla u_{n}(t)\right|^{2} d x+\mathcal{L}_{N}(\Omega) \max (|\underline{\rho}|,|\bar{\rho}|)^{2}\right) \\
& +\mathcal{L}_{N}(\Omega)^{\frac{1}{2}} \max (|\underline{\rho}|,|\bar{\rho}|)\left(\left\|\frac{d u_{n}^{+}}{d t}(t)\right\|_{X}+\left\|F_{n}\left(t, u_{n}(t)\right)\right\|_{X}\right) .
\end{aligned}
$$


Hence

$$
\begin{aligned}
\left(\gamma-\frac{C_{\text {trace }} \nu}{2}\right) \int_{\Omega}\left|\nabla u_{n}(t)\right|^{2} d x & \leq \frac{C_{\text {trace }}}{2 \nu} \sup _{n \in \mathbb{N}}\left\|\phi_{n}\right\|_{L_{\mathcal{H}_{N-1}}^{2}(\partial \Omega)^{2}}+\frac{C_{\text {trace }} \nu}{2} \mathcal{L}_{N}(\Omega) \max (|\underline{\rho}|,|\bar{\rho}|)^{2} \\
& +\mathcal{L}_{N}(\Omega)^{\frac{1}{2}} \max (|\underline{\rho}|,|\bar{\rho}|)\left(\left\|\frac{d u_{n}^{+}}{d t}(t)\right\|_{X}+\left\|F_{n}\left(t, u_{n}(t)\right)\right\|_{X}\right) .
\end{aligned}
$$

To conclude, it suffices to prove that

$$
\sup _{n \in \mathbb{N}}\left(\left\|\frac{d u_{n}^{+}}{d t}(t)\right\|_{X}+\left\|F_{n}\left(t, u_{n}(t)\right)\right\|_{X}\right)<+\infty .
$$

Indeed, from (27), (18), and the compactness embedding $H^{1}(\Omega) \rightarrow L^{2}(\Omega)$ we will conclude the compactness of the set $E_{t}$ for each $\left.\left.t \in\right] 0, T\right]$. For proving $[28$, we establish successively

$$
\begin{aligned}
& \sup _{n \in \mathbb{N}}\left\|F_{n}\left(t, u_{n}(t)\right)\right\|_{X}<+\infty ; \\
& \sup _{n \in \mathbb{N}}\left\|\frac{d u_{n}^{+}}{d t}(t)\right\|_{X}<+\infty .
\end{aligned}
$$

Proof of 29). This estimate follows straightforwardly from (18), 23), (24), and hypothesis $\left(\mathrm{H}_{6}\right)$. Proof of (30). By applying Proposition 3.3. we deduce that there exists $C_{n}$ such that

$$
\left\|\frac{d u_{n}^{+}}{d t}(t)\right\|_{X} \leq C_{n}+\left(C_{n}+\frac{1}{t}\right) \int_{0}^{T}\left\|\frac{d u_{n}}{d t}(t)\right\|_{X} d t .
$$

From (9), the uniform Lipchitz condition (13) together with $\left(\mathrm{H}_{4}\right),\left(\mathrm{H}_{5}\right)$, and $\left(\mathrm{H}_{6}\right)$, we infer that $\sup _{n \in \mathbb{N}} C_{n}<+\infty$. Hence 30 follows from 19$]$.

Step 3. We assert that $\frac{d u_{n}}{d t} \rightarrow \frac{d u}{d t}$ weakly in $L^{2}(0, T, X)$ for a non relabeled subsequence, and that $\underline{\rho} \leq u \leq \bar{\rho}$. The first claim is a straightforward consequence of $(19)$ and Step 2. The second one follows easily from inequality $\underline{\rho} \leq u_{n} \leq \bar{\rho}$ and $u_{n} \rightarrow u$ in $C([0, T], X)$.

Step 4. We prove that $u$ is the unique solution of $(\mathcal{P})$. From Step 2, there exists $u \in C([0, T], X)$ and a (non relabeled) subsequence such that $u_{n} \rightarrow u$ in $C([0, T], X)$. To simplify the notation, we still write $G_{n}(t)=F_{n}\left(t, u_{n}(t)\right)$ and we use the subsequence obtained in Step 3, that we do not relabel. According to the Fenchel extremality condition (see [8, Proposition 9.5.1]) $\left(\mathcal{P}_{n}\right.$ ) is equivalent to

$$
\Phi_{n}\left(u_{n}(t)\right)+\Phi_{n}^{*}\left(G_{n}(t)-\frac{d u_{n}}{d t}(t)\right)+\left\langle\frac{d u_{n}}{d t}(t)-G_{n}(t), u_{n}(t)\right\rangle=0
$$

for a.e. $t \in(0, T)$ (together with the initial condition that we do not write), which is also equivalent to

$$
\int_{0}^{T}\left[\Phi_{n}\left(u_{n}(t)\right)+\Phi_{n}^{*}\left(G_{n}(t)-\frac{d u_{n}}{d t}(t)\right)+\left\langle\frac{d u_{n}}{d t}(t)-G_{n}(t), u_{n}(t)\right\rangle\right] d t=0 .
$$

Note that equivalence above is due to the Legendre-Fenchel inequality which asserts that inequality $\Phi_{n}\left(u_{n}(t)\right)+\Phi_{n}^{*}\left(G_{n}(t)-\frac{d u_{n}}{d t}(t)\right)+\left\langle\frac{d u_{n}}{d t}(t)-G_{n}(t), u_{n}(t)\right\rangle \geq 0$ for a.e. $t \in(0, T)$, is always true (see [8, Remark 9.5.1]). Therefore, $\left(\mathcal{P}_{n}\right)$ is equivalent to

$$
\int_{0}^{T}\left[\Phi_{n}\left(u_{n}(t)\right)+\Phi^{*}\left(G_{n}(t)-\frac{d u_{n}}{d t}(t)\right)+\frac{d}{d t} \frac{1}{2}\left\|u_{n}(t)\right\|^{2}-\left\langle G_{n}(t), u_{n}(t)\right\rangle\right] d t=0,
$$

or, equivalently, to

$$
\int_{0}^{T}\left[\Phi_{n}\left(u_{n}(t)\right)+\Phi_{n}^{*}\left(G_{n}(t)-\frac{d u_{n}}{d t}(t)\right)\right] d t+\frac{1}{2}\left(\left\|u_{n}(T)\right\|^{2}-\left\|u_{n}^{0}\right\|^{2}\right)-\int_{0}^{T}\left\langle G_{n}(t), u_{n}(t)\right\rangle d t=0 .
$$

From hypothesis $\left(\mathrm{H}_{3}\right)$ we have

$$
\left\|u_{n}^{0}\right\|_{X} \rightarrow\left\|u^{0}\right\|_{X}
$$

Combining $u_{n}(T)=u_{n}^{0}+\int_{0}^{T} \frac{d u_{n}}{d t}(t) d t$ with $\frac{d u_{n}}{d t} \rightarrow \frac{d u}{d t}$ in $L^{2}(0, T, X)$, we infer that

$$
\liminf _{n \rightarrow+\infty}\left\|u_{n}(T)\right\|^{2} \geq\|u(T)\|^{2} .
$$


We postpone the proof of the following convergence at the last step of the proof (see Lemma 4.2)

$$
G_{n} \rightarrow G \text { weakly in } L^{2}(0, T, X)
$$

where $G(t)=F(t, u(t))$ and $F(t, u(t))(x)=r(t, x) \cdot g(u(t, x))+q(t, x)$. Passing to the limit in (31), from 32 , 33), (34), Step 3, and Lemma 4.1, we obtain ${ }^{5}$

$$
\int_{0}^{T}\left[\Phi(u(t))+\Phi^{*}\left(G(t)-\frac{d u}{d t}(t)\right)\right] d t+\frac{1}{2}\left(\|u(T)\|^{2}-\left\|u^{0}\right\|^{2}\right)-\int_{0}^{T}\langle G(t), u(t)\rangle d t \leq 0
$$

or equivalently

$$
\int_{0}^{T}\left[\Phi(u(t))+\Phi^{*}\left(G(t)-\frac{d u}{d t}(t)\right)+\left\langle\frac{d u}{d t}(t)-G(t), u(t)\right\rangle\right] d t \leq 0 .
$$

But from the Legendre-Fenchel inequality we have $\Phi(u(t))+\Phi^{*}\left(G(t)-\frac{d u}{d t}(t)\right)+\left\langle\frac{d u}{d t}(t)-G(t), u(t)\right\rangle \geq$ 0 , so that 35 yields that for a.e. $t \in(0, T), \Phi(u(t))+\Phi^{*}\left(G(t)-\frac{d u}{d t}(t)\right)+\left\langle\frac{d u}{d t}(t)-G(t), u(t)\right\rangle=0$ which is, according to [8, Proposition 9.5.1], equivalent to

$$
\frac{d u}{d t}(t)+\partial \Phi(u(t)) \ni G(t) \text { for a.e. } t \in(0, T) .
$$

We have already proved that $\underline{\rho} \leq u_{0} \leq \bar{\rho}$ in Step 3. It remains to establish that $u_{0} \in \operatorname{dom}(\Phi)$. From $\left(\mathrm{H}_{1}\right)$ and $\left(\mathrm{H}_{2}\right)$, we infer that

$$
\Phi\left(u_{0}\right) \leq \liminf _{n \rightarrow+\infty} \Phi_{n}\left(u_{n}^{0}\right) \leq \sup _{n \in \mathbb{N}} \Phi_{n}\left(u_{n}^{0}\right)<+\infty,
$$

which prove the thesis. For the proof of uniqueness of

$$
(\mathcal{P})\left\{\begin{array}{l}
\frac{d u}{d t}(t)+\partial \Phi(u(t)) \ni F(t, u(t)) \text { for a.e. } t \in(0, T) \\
u(0)=u^{0}, \quad a \leq u^{0} \leq b, \quad u^{0} \in H^{1}(\Omega)
\end{array}\right.
$$

it is enough to reproduce the proof of uniqueness of Theorem 2.1, with a Lipschitz constant for $F$ given by $L(t)=\|r\|_{L^{\infty}\left([0, T] \times \mathbb{R}^{N}, \mathbb{R}^{l}\right)} L_{[\rho, \bar{\rho}]}$. Since every subsequence of the subsequence of $\left(u_{n}\right)_{n \in \mathbb{N}}$ obtained above converges to the same limit $\bar{u}$ in $C([0, T], X)$, the sequence $\left(u_{n}\right)_{n \in \mathbb{N}}$ converges to $u$ in $C([0, T], X)$. Idem for the sequence $\left(\frac{d u_{n}}{d t}\right)_{n \in \mathbb{N}}$ which converges to $\frac{d u}{d t}$ weakly in $L^{2}(0, T, X)$.

Step 5. We show that if $\Phi\left(u_{n}^{0}\right) \rightarrow \Phi\left(u^{0}\right), r_{n} \rightarrow r$ strongly in $L^{2}\left(0, T, L^{2}\left(\Omega, \mathbb{R}^{l}\right)\right)$ and $q_{n} \rightarrow q$ strongly in $L^{2}(0, T, X)$, then $\frac{d u_{n}}{d t} \rightarrow \frac{d u}{d t}$ strongly in $L^{2}(0, T, X)$. From Step 3 and Step 4 we have $\frac{d u_{n}}{d t} \rightarrow \frac{d u}{d t}$ weakly in $L^{2}(0, T, X)$, hence it suffices to establish that $\left\|\frac{d u_{n}}{d t}\right\|_{L^{2}(0, T, X)}^{2} \rightarrow\left\|\frac{d u}{d t}\right\|_{L^{2}(0, T, X)}^{2}$ to prove the claim. By repeating the proof of Lemma 4.2 below under the hypotheses of strong convergence of $r_{n}$ and $q_{n}$ to $r$ and $q$ respectively, it is easily seen that $G_{n}$ strongly converges to $G$ in $L^{2}(0, T, X)$. Therefore, passing to the limit on 21 , and since $\Phi_{n} \stackrel{M}{\rightarrow} \Phi$, we deduce that

$$
\begin{aligned}
\limsup _{n \rightarrow+\infty} \int_{0}^{T}\left\|\frac{d u_{n}}{d t}(t)\right\|_{X}^{2} d t & =-\liminf _{n \rightarrow+\infty} \Phi_{n}\left(u_{n}(T)\right)+\Phi\left(u^{0}\right)+\int_{0}^{T}\left\langle F(t, u(t)), \frac{d u}{d t}(t)\right\rangle d t \\
& \leq-\Phi(u(T))+\Phi\left(u^{0}\right)+\int_{0}^{T}\left\langle F(t, u(t)), \frac{d u}{d t}(t)\right\rangle d t \\
& =\int_{0}^{T}\left\|\frac{d u}{d t}(t)\right\|_{X}^{2} d t .
\end{aligned}
$$

The conclusion follows from the lower semicontinuity of the convex function $\zeta \mapsto\|\zeta\|_{L^{2}(0, T, X)}^{2}$ in $L^{2}(0, T, X)$.

Last step. We establish the convergence (34) invoked in Step 4.

Lemma 4.2. The functional $G_{n}=F_{n}\left(\cdot, u_{n}\right)$ weakly converges in $L^{2}(0, T, X)$ to $G$ defined by $G(t)=$ $F(t, u(t))$ where $F(t, u(t))(x)=r(t, x) \cdot g(u(t, x))+q(t, x)$.

\footnotetext{
${ }^{5}$ From hypotheses $\left(\mathrm{H}_{1}\right)\left(\mathrm{H}_{2}\right)$ and $\left(\mathrm{H}_{3}\right)$ the sequence $\left(\Phi_{n}\right)_{n \in \mathbb{N}}, \Phi$, is clearly uniformly proper.
} 
Proof. Recall that $G_{n}(t)=H_{n}(t)+q_{n}(t)$ where

$$
H_{n}(t)(x)=r_{n}(t, x) \cdot g_{n}\left(u_{n}(t, x)\right) .
$$

Hence, since $q_{n} \rightarrow q$ in $L^{2}(0, T, X)$, it remains to prove that $H_{n} \rightarrow H$ in $L^{2}(0, T, X)$ where $H(t)(x)=$ $r(t, x) \cdot g(u(t, x))$. According to 130 in the interval $I=[\underline{\rho}, \bar{\rho}]$, we have ${ }^{6}$

$$
\left\|g_{n}\left(u_{n}(t)\right)-g(u(t))\right\|_{L^{2}\left(\Omega, \mathbb{R}^{l}\right)} \leq L_{I}\left\|u_{n}(t)-u(t)\right\|_{X}+\left\|g_{n}(u(t))-g(u(t))\right\|_{L^{2}\left(\Omega, \mathbb{R}^{l}\right)} .
$$

Hence

$$
\int_{0}^{T}\left\|g_{n}\left(u_{n}(t)\right)-g(u(t))\right\|_{L^{2}\left(\Omega, \mathbb{R}^{l}\right)}^{2} d t \leq 2 L_{I}^{2} \int_{0}^{T}\left\|u_{n}(t)-u(t)\right\|_{X}^{2} d t+\int_{0}^{T}\left\|g_{n}(u(t))-g(u(t))\right\|_{L^{2}\left(\Omega, \mathbb{R}^{l}\right)}^{2} d t .
$$

On the other hand, from $[13)$, and hypothesis $\left(\mathrm{H}_{4}\right)$ we clearly deduce that $\left|g_{n}(\zeta)\right| \leq C(1+|\zeta|)$ where $C$ is a nonnegative constant depending only on $L_{I}$ and $g(0)$. Consequently, applying the Lebesgue dominated convergence theorem and $\left(\mathrm{H}_{4}\right)$, we infer that

$$
\lim _{n \rightarrow+\infty} \int_{0}^{T}\left\|g_{n}(u(t))-g(u(t))\right\|_{L^{2}\left(\Omega, \mathbb{R}^{l}\right)}^{2} d t=0 .
$$

Passing to the limit in $(36)$ we deduce that $g_{n}\left(u_{n}(\cdot)\right) \rightarrow g(u(\cdot))$ strongly in $L^{2}\left(0, T, L^{2}\left(\Omega, \mathbb{R}^{l}\right)\right)$. The conclusion of Lemma 4.2 follows from the fact that $r_{n} \rightarrow r$ weakly in $L^{2}\left(0, T, L^{2}\left(\Omega, \mathbb{R}^{l}\right)\right)$.

The proof of Theorem 4.1 is complete.

In some cases, we can specify the domain of the limit functional $\Phi$ as in the proposition below.

Proposition 4.1. Let denote by $\operatorname{dom}(\Phi)$ the domain of the Mosco-limit $\Phi$ of the sequence $\left(\Phi_{n}\right)_{n \in \mathbb{N}}$. Then we have

i) if $\alpha_{n}=\alpha$ for all $n \in \mathbb{N}$, then $\operatorname{dom}(\Phi) \subset H^{1}(\Omega)$,

ii) if $\liminf _{n \rightarrow \infty} \beta_{n}<+\infty, a_{0, n} \rightarrow a_{0}$ for the $\sigma\left(L_{\mathcal{H}_{N-1}}^{\infty}(\partial \Omega), L_{\mathcal{H}_{N-1}}^{1}(\partial \Omega)\right)$ topology, and $h_{n} \rightarrow h$ weakly in $L_{\mathcal{H}_{N-1}}^{2}(\partial \Omega)$, then $H^{1}(\Omega) \subset \operatorname{dom}(\Phi)$.

Proof. Let us establish the first assertion. Let $v \in \operatorname{dom}(\Phi)$, then from $\left(H_{1}\right)$, there exists $v_{n} \rightarrow v$ strongly in $L^{2}(\Omega)$ such that $\lim _{n \rightarrow+\infty} \Phi_{n}\left(v_{n}\right)=\Phi(v)<+\infty$. From the uniform lower growth condition of $\Phi_{n}$, and hypotheses $\left(\mathrm{H}_{1}\right)$ and $\left(\mathrm{H}_{2}\right)$ we infer that for any $\nu>0$,

$$
\begin{aligned}
\alpha \int_{\Omega}\left|\nabla v_{n}(t)\right|^{2} d x & \leq \sup _{n \in \mathbb{N}} \Phi_{n}\left(v_{n}\right)+\left\|h_{n}\right\|_{L_{\mathcal{H}_{N-1}}^{2}(\partial \Omega)}\left\|v_{n}\right\|_{L_{\mathcal{H}_{N-1}}^{2}}(\partial \Omega) \\
& \leq \sup _{n \in \mathbb{N}} \Phi_{n}\left(v_{n}\right)+\frac{C_{\text {trace }}}{2 \nu}\left\|h_{n}\right\|_{L_{\mathcal{H}_{N-1}}^{2}(\partial \Omega)}^{2}+\frac{C_{\text {trace }} \nu}{2}\left\|v_{n}\right\|_{H^{1}(\Omega)}^{2} .
\end{aligned}
$$

Hence choosing $\nu$ such that $\alpha-\frac{1}{2} C_{\text {trace }} \nu>0$, we obtain for some constant $C>0$,

$$
\int_{\Omega}\left|\nabla v_{n}(t)\right|^{2} d x \leq C\left(1+\left\|v_{n}\right\|_{L^{2}(\Omega)}^{2}\right)
$$

and, finally, from $\left(\mathrm{H}_{3}\right)$

$$
\sup _{n \in \mathbb{N}}\left\|v_{n}\right\|_{H^{1}(\Omega)}<+\infty
$$

Therefore, there exists a subsequence, that we do not relabel, and $w \in H^{1}(\Omega)$ satisfying $v_{n} \rightarrow w$ weakly in $H^{1}(\Omega)$ and strongly in $L^{2}(\Omega)$. Hence $v=w \in H^{1}(\Omega)$.

On the other hand, for $v \in H^{1}(\Omega)$, according to $\left(H_{1}\right)$ and the growth condition, one has

$$
\Phi(v) \leq \liminf _{n \rightarrow+\infty} \Phi_{n}(v) \leq \liminf _{n \rightarrow+\infty} \beta_{n}\left(1+\int_{\Omega}|\nabla v|^{2} d x\right)+\frac{1}{2} \int_{\partial \Omega} a_{0} v^{2} d \mathcal{H}_{N-1}-\int_{\partial \Omega} h v d \mathcal{H}_{N-1}<+\infty,
$$

from which we infer the second assertion.

\footnotetext{
${ }^{6}$ To simplify the notation we write $g_{n}\left(u_{n}(t)\right)$ for the function $x \mapsto g_{n}\left(u_{n}(t, x)\right)$.
} 
For each $n \in \mathbb{N}$ let us write $\Phi_{n}: L^{2}(\Omega) \rightarrow \mathbb{R} \cup\{+\infty\}$ as follows:

$$
\Phi_{n}(u)=\left\{\begin{array}{cl}
\widetilde{\Phi}_{n}(u)+\frac{1}{2} \int_{\partial \Omega} a_{0, n} u^{2} d \mathcal{H}_{N-1}-\int_{\partial \Omega} h_{n} u d \mathcal{H}_{N-1} & \text { if } u \in H^{1}(\Omega) \\
+\infty & \text { otherwise, }
\end{array}\right.
$$

where $\widetilde{\Phi}_{n}: H^{1}(\Omega) \rightarrow \mathbb{R}_{+}$is defined by $\widetilde{\Phi}_{n}(u)=\int_{\Omega} W_{n}(x, \nabla u(x)) d x$. The following result gives sufficient conditions for the Mosco-convergence of $\left(\Phi_{n}\right)_{n \in \mathbb{N}}$ when we assume that $\widetilde{\Phi}_{n} \Gamma$-converges to $\widetilde{\Phi}$ with respect to the $L^{2}(\Omega)$ topology.

Proposition 4.2. Assume that

$\left(\mathrm{H}_{1}^{\prime}\right) \bullet$ there exist $\alpha>0$ and $\beta>0$ such that the sequence $\left(W_{n}\right)_{n \in \mathbb{N}}$ satisfies $\left(\mathrm{D}_{1, n}\right)$ with $\alpha_{n}=\alpha$ and $\beta_{n}=\beta$ for all $n \in \mathbb{N}$;

- $\widetilde{\Phi}_{n} \Gamma$-converges to $\widetilde{\Phi}$ when $H^{1}(\Omega)$ is equipped with the strong convergence of $L^{2}(\Omega)$;

- $a_{0, n} \rightarrow a_{0}$ strongly in $L_{\mathcal{H}_{N-1}}^{\infty}(\partial \Omega)$;

- $h_{n} \rightarrow h$ strongly in $L_{\mathcal{H}_{N-1}}^{2}(\partial \Omega)$.

Then $\Phi_{n} \stackrel{M}{\rightarrow} \Phi$ where $\Phi: L^{2}(\Omega) \rightarrow \mathbb{R} \cup\{+\infty\}$ is given by

$$
\Phi(u)=\left\{\begin{array}{cl}
\widetilde{\Phi}(u)+\frac{1}{2} \int_{\partial \Omega} a_{0} u^{2} d \mathcal{H}_{N-1}-\int_{\partial \Omega} h u d \mathcal{H}_{N-1} & \text { if } u \in H^{1}(\Omega) \\
+\infty & \text { otherwise. }
\end{array}\right.
$$

Proof. The proof fall into two steps.

Step 1. Let $v_{n} \rightarrow v$ weakly in $L^{2}(\Omega)$, we establish that $\Phi(v) \leq \liminf _{n \rightarrow+\infty} \Phi_{n}\left(v_{n}\right)$.

We assume that $\liminf _{n \rightarrow+\infty} \Phi_{n}\left(v_{n}\right)<+\infty$ and we reason with various subsequences that we do not relabel. Moreover $C$ denotes various positive constants. From $\left(\mathrm{H}_{1}^{\prime}\right)$ the uniform lower bound of $W_{n}$, and the continuity of the trace operator, we have, for $\nu>0$,

$$
\begin{aligned}
\alpha \int_{\Omega}\left|\nabla v_{n}\right|^{2} d x & \leq C+\left\|h_{n}\right\|_{L_{\mathcal{H}_{N-1}}^{2}(\partial \Omega)}\left\|v_{n}\right\|_{L_{\mathcal{H}_{N-1}}^{2}(\partial \Omega)} \\
& \leq C+\frac{C_{\text {trace }}}{2 \nu}\left\|h_{n}\right\|_{L_{\mathcal{H}_{N-1}}^{2}(\partial \Omega)}^{2}+\frac{C_{\text {trace }} \nu}{2}\left\|v_{n}\right\|_{H^{1}(\Omega)}^{2} .
\end{aligned}
$$

Hence

$$
\left(\alpha-\frac{C_{\text {trace }} \nu}{2}\right) \int_{\Omega}\left|\nabla v_{n}\right|^{2} d x \leq C\left(1+\left\|v_{n}\right\|_{H^{1}(\Omega)}^{2}\right) .
$$

Therefore, choosing $\nu<\frac{2 \alpha}{C_{\text {trace }}}$, we deduce that

$$
\sup _{n \in \mathbb{N}}\left\|v_{n}\right\|_{H^{1}(\Omega)}<+\infty .
$$

Consequently, there exist a subsequence and $w \in H^{1}(\Omega)$ such that $v_{n} \rightarrow w$ weakly in $H^{1}(\Omega)$ and strongly in $L^{2}(\Omega)$. Thus $w=v$ so that $v \in H^{1}(\Omega)$ and $v_{n} \rightarrow v$ strongly in $L^{2}(\Omega)$. According to $\left(\mathrm{H}_{1}^{\prime}\right)$, we infer that

$$
\widetilde{\Phi}(v) \leq \liminf _{n \rightarrow+\infty} \widetilde{\Phi}_{n}\left(v_{n}\right)
$$

On the other hand

$$
\begin{aligned}
\int_{\partial \Omega} a_{0, n} v_{n}^{2} d \mathcal{H}_{N-1} & =\int_{\partial \Omega}\left(a_{0, n}-a_{0}\right) v_{n}^{2} d \mathcal{H}_{N-1}+\int_{\partial \Omega} a_{0} v_{n}^{2} d \mathcal{H}_{N-1} \\
& \geq-\left\|a_{0, n}-a_{0}\right\|_{L_{\mathcal{H}_{N-1}}^{\infty}(\partial \Omega)} \sup _{n \in \mathbb{N}} \int_{\partial \Omega} v_{n}^{2} d \mathcal{H}_{N-1}+\int_{\partial \Omega} a_{0} v_{n}^{2} d \mathcal{H}_{N-1} .
\end{aligned}
$$

According to the weak continuity of the trace operator from $H^{1}(\Omega)$ into $L_{\mathcal{H}_{N-1}}^{2}(\partial \Omega)$ and to the lower semicontinuity of the map $w \mapsto \int_{\partial \Omega} a_{0} w^{2} d \mathcal{H}_{N-1}$, we infer that

$$
\int_{\partial \Omega} a_{0} v^{2} d \mathcal{H}_{N-1} \leq \liminf _{n \rightarrow+\infty} \int_{\partial \Omega} a_{0, n} v_{n}^{2} d \mathcal{H}_{N-1}
$$


Finally, since $h_{n} \rightarrow h$ strongly in $L_{\mathcal{H}_{N-1}}^{2}(\partial \Omega)$, and $v_{n} \rightarrow v$ weakly in $L_{\mathcal{H}_{N-1}}^{2}(\partial \Omega)$, we have

$$
\lim _{n \rightarrow+\infty} \int_{\partial \Omega} h_{n} v_{n} d \mathcal{H}_{N-1}=\int_{\partial \Omega} h v d \mathcal{H}_{N-1} .
$$

The proof of the claim is obtained by collecting (37), (38), and (39).

Step 2. Assume that $\Phi(v)<+\infty$. We prove that there exists a sequence $\left(v_{n}\right)_{n \in \mathbb{N}}$ strongly converging to $v$ in $L^{2}(\Omega)$ such that $\limsup _{n \rightarrow+\infty} \Phi_{n}\left(v_{n}\right) \leq \Phi(v)$.

Since $\Phi(v)<+\infty$, we infer that $v \in H^{1}(\Omega)$, and, according to hypothesis $\left(\mathrm{H}_{1}^{\prime}\right)$, there exists a sequence $\left(w_{n}\right)_{n \in \mathbb{N}}$ in $H^{1}(\Omega)$ strongly converging to $v$ in $L^{2}(\Omega)$, such that

$$
\lim _{n \rightarrow+\infty} \widetilde{\Phi}_{n}\left(w_{n}\right)=\Phi(v) .
$$

By using the well known De Giorgi slicing method, (it is precisely at this point that we use the uniform growth condition), we can modify $w_{n}$ into a function $v_{n}$ in $H^{1}(\Omega)$ satisfying $v_{n}=v$ on $\partial \Omega$ and

$$
\limsup _{n \rightarrow+\infty} \widetilde{\Phi}_{n}\left(v_{n}\right) \leq \widetilde{\Phi}(v)
$$

(see proof of [8, Corollary 11.2.1]). Then clearly $\lim \sup _{n \rightarrow+\infty} \Phi_{n}\left(v_{n}\right) \leq \Phi(v)$, which proof the claim.

Proposition 4.2 leads straight to the following corollary of Theorem 4.1 which is applied in Theorem 5.1 below.

Corollary 4.1. Under hypotheses of Theorem 4.1 where $\left(\mathrm{H}_{1}\right)$ is replaced by $\left(\mathrm{H}_{1}^{\prime}\right)$, the same conclusions hold.

Remark 4.1. We can, in some sense, justify our convention which consists to see the functional

$$
\widetilde{\Phi}(u)=\left\{\begin{array}{cl}
\int_{\Omega} W(x, \nabla u(x)) d x & \text { if } u \in H_{\Gamma}^{1}(\Omega) \\
+\infty & \text { otherwise }
\end{array}\right.
$$

as a particular case of

$$
\Phi(u)=\left\{\begin{array}{cl}
\int_{\Omega} W(x, \nabla u(x)) d x+\frac{1}{2} \int_{\partial \Omega} a_{0} u^{2} d \mathcal{H}_{N-1}-\int_{\partial \Omega} h u d \mathcal{H}_{N-1} & \text { if } u \in H^{1}(\Omega) \\
+\infty & \text { otherwise }
\end{array}\right.
$$

with $h=0$ and $a_{0}(x)= \begin{cases}0 & \text { if } x \in \partial \Omega \backslash \Gamma \\ +\infty & \text { if } x \in \Gamma\end{cases}$

For this purpose we apply suitably Theorem 4.1 Set $h_{n}=0$ and $a_{0, n}(x)=\left\{\begin{array}{ll}0 & \text { if } x \in \partial \Omega \backslash \Gamma \\ n & \text { if } x \in \Gamma\end{array}\right.$. We have

$$
\Phi_{n}(u)=\left\{\begin{array}{cl}
\int_{\Omega} W(x, \nabla u(x)) d x+\frac{n}{2} \int_{\Gamma} u^{2} d \mathcal{H}_{N-1} & \text { if } u \in H^{1}(\Omega) \\
+\infty & \text { otherwise. }
\end{array}\right.
$$

On the other hand, set $F_{n}=F$ and $u_{0}^{n}=u_{0}, \quad \underline{\rho} \leq u_{0} \leq \bar{\rho}, \quad u_{0} \in \overline{\operatorname{dom}(D \Phi)}$. The conditions $a_{0, n} \rho \leq h_{n} \leq a_{0, n} \bar{\rho}$ on $\partial \Omega$ become $\underline{\rho} \leq 0 \leq \bar{\rho}$. We claim that $\Phi_{n}$ Mosco-converges to $\widetilde{\Phi}$.

Consider a sequence $\left(v_{n \in \mathbb{N}}\right)_{n \in \mathbb{N}}$ satisfying $v_{n} \rightarrow v$ strongly in $L^{2}(\Omega)$ and $\liminf \operatorname{in}_{n \rightarrow+\infty} \Phi_{n}\left(v_{n}\right)<+\infty$. In what follows, we reason with various subsequences that we do not relabel. From

we infer that

$$
\sup _{n \in \mathbb{N}} \frac{n^{2}}{2} \int_{\Gamma} v_{n}^{2} d \mathcal{H}_{N-1}<+\infty
$$

$$
v_{n} \rightarrow 0 \text { strongly in } L_{\mathcal{H}_{N-1}}^{2}(\Gamma)
$$


On the other hand, from

$$
\sup _{n \in \mathbb{N}} \int_{\Omega} W\left(x, \nabla v_{n}(x)\right) d x<+\infty
$$

and the lower bound condition of $W$, we deduce that the sequence $\left(v_{n}\right)_{n \in \mathbb{N}}$ is bounded in $H^{1}(\Omega)$ (recall that $v_{n} \rightarrow v$ in $\left.L^{2}(\Omega)\right)$. Therefore $v_{n} \rightarrow v$ weakly in $H^{1}(\Omega)$, and, according to the continuity of the trace operator from $H^{1}(\Omega)$ into $L_{\mathcal{H}_{N-1}}^{2}(\partial \Omega), v_{n} \rightarrow v$ weakly in $L_{\mathcal{H}_{N-1}}^{2}(\Gamma)$. From 40 we infer that $v=0$ in $\Gamma$, hence $v \in H_{\Gamma}^{1}(\Omega)$ and $\widetilde{\Phi}(v)=\int_{\Omega} W(x, \nabla v(x)) d x$. Since for all $n \in \mathbb{N}$,

$$
\int_{\Omega} W(x, \nabla v(x)) d x \leq \Phi_{n}\left(v_{n}\right)
$$

we deduce that $\widetilde{\Phi}(v) \leq \liminf _{n \rightarrow+\infty} \Phi_{n}\left(v_{n}\right)$.

Take now $v \in H_{\Gamma}^{1}(\Omega)$ (otherwise we have nothing to prove), and set $v_{n}=v$. Since $\Phi(v)=\Phi_{n}(v)$, we have $\lim _{n \rightarrow+\infty} \Phi_{n}\left(v_{n}\right)=\widetilde{\Phi}(v)$, which proves the claim.

Since all other conditions of Theorem 4.1 are fulfilled, we deduce that problem $\left(\mathcal{P}_{n}\right)$ with mixed Dirichlet-Neumann boundary conditions

$$
\left(\mathcal{P}_{n}\right)\left\{\begin{array}{l}
\left.\left.\frac{d u}{d t}(t)-\operatorname{div} D_{\xi} W(\cdot, \nabla u(t))=F(t, u(t)) \text { for all } t \in\right] 0, T\right] \quad\left(\text { equality in } L^{2}(\Omega)\right) \\
u(0)=u_{0}, \quad \underline{\rho} \leq u_{0} \leq \bar{\rho}, \quad u_{0} \in \overline{\operatorname{dom}(D \Phi)}, \\
\left.\left.n u(t)+D_{\xi} W(\cdot, \nabla u(t)) \cdot \mathbf{n}=0 \text { on } \partial \Omega \text { for all } t \in\right] 0, T\right] .
\end{array}\right.
$$

converges in the sense of Theorem 4.1. to problem $(\mathcal{P})$ with homogeneous Dirichlet-Neumann boundary conditions

$$
(\mathcal{P})\left\{\begin{array}{l}
\left.\left.\frac{d u}{d t}(t)-\operatorname{div} D_{\xi} W(\cdot, \nabla u(t))=F(t, u(t)) \text { for all } t \in\right] 0, T\right] \quad\left(\text { equality in } L^{2}(\Omega)\right) \\
u(0)=u_{0}, \quad \underline{\rho} \leq u_{0} \leq \bar{\rho}, \quad u_{0} \in \overline{\operatorname{dom}(D \Phi)}, \\
u(t)=0 \text { on } \Gamma \text { for all } t \in] 0, T], \\
\left.\left.D_{\xi} W(\cdot, \nabla u(t)) \cdot \mathbf{n}=0 \text { on } \partial \Omega \backslash \Gamma \text { for all } t \in\right] 0, T\right] .
\end{array}\right.
$$

\section{Application to stochastic homogenization}

The behavior of heterogeneous media in physics or mechanics has been thoroughly analyzed from a mathematical perspective through the framework of homogenization. In this context, diffusion problems with periodic heterogeneities are now well understood, and diffusion in random media has been fairly well analyzed in [17] and [8, Sections 17.4.4, 17.4.5], where the diffusion operator is the subdifferential of a random energy.

By contrast, homogenization of reaction-diffusion problems modeling for example biological invasion in the context of food limited population dynamics, does not seem to be addressed. The interplay between environment heterogeneities in the individual evolution of propagation species, plays an essential role. Indeed, empirical observations suggest that growth rates, or various thresholds which appear in the models, are mostly influenced by the environment, and vary in each small habitats (forests, marshes, hedges, etc.). Most of the time, these heterogeneities appear very small compared with the dimension of the domain, and statistically, are homogeneously distributed. Therefore both diffusion and reaction parts in the problems modeling the propagation, present random coefficients and a small parameter $\varepsilon$ which accounts for the dimension of heterogeneities. To identify the effective coefficients (effective growth rate, various effective thresholds etc.), the purpose of this section is to describe the equivalent homogenized problem when $\varepsilon$ goes to zero. The procedure consists in applying Theorem 4.1 in Section 4. 
5.1. Probabilistic setting. For any topological space $\mathcal{X}$, we denote by $\mathcal{B}(\mathcal{X})$ its Borel $\sigma$-field, and we return to the basic concepts of [8, Section 12.4.3] (see also references therein) concerning ergodic dynamic systems. Let $(\Sigma, \mathcal{A}, \mathbf{P})$ be a probability space. Let $\left(T_{z}\right)_{z \in \mathbb{Z}^{N}}$ be a group of $\mathbf{P}$-preserving transformations on $\Sigma$, i.e., for all $z \in \mathbb{Z}^{N}$, the map $T_{z}: \Sigma \rightarrow \Sigma$ is $\mathcal{A}$ measurable and satisfies $T_{z} \# \mathbf{P}=\mathbf{P}$, where we use the standard notation $T_{z} \# \mathbf{P}$ to denote the image measure (or push forward) of $\mathbf{P}$ by $T_{z}$. We denote by $\mathcal{I}$ the $\sigma$-algebra of invariant sets of $\mathcal{A}$ by the group $\left(T_{z}\right)_{z \in \mathbb{Z}^{N}}$ and, for every $\mathbf{h}$ in the space $L_{\mathbf{P}}^{1}(\Sigma)$ of $\mathbf{P}$-integrable functions, by $\mathbf{E}^{\mathcal{I}} \mathbf{h}$ the conditional expectation of $\mathbf{h}$ with respect to $\mathcal{I}$, i.e., the unique $\mathcal{I}$-measurable function in $L_{\mathbf{P}}^{1}(\Sigma)$ satisfying for every $E \in \mathcal{I}$

$$
\int_{E} \mathbf{E}^{\mathcal{I}} \mathbf{h}(\omega) d \mathbf{P}(\omega)=\int_{E} \mathbf{h}(\omega) d \mathbf{P}(\omega) .
$$

If $\mathcal{I}$ is made up of sets with probability 0 or 1 , the discrete dynamical system $\left(\Sigma, \mathcal{A}, \mathbf{P},\left(T_{z}\right)_{z \in \mathbb{Z}^{N}}\right)$ is said to be ergodic. Under this condition, we have $\mathbf{E}^{\mathcal{I}} \mathbf{h}=\mathbf{E h}$ where $\mathbf{E h}=\int_{\Sigma} \mathbf{h}(\omega) d \mathbf{P}(\omega)$ is the mathematical expectation of $\mathbf{h}$.

A sufficient condition to ensure ergodicity is the so called mixing condition which expresses an asymptotic independence: for all sets $E$ and $F$ of $\mathcal{A}$

$$
\lim _{|z| \rightarrow+\infty} \mathbf{P}\left(T_{z} E \cap F\right)=\mathbf{P}(E) \mathbf{P}(F) .
$$

Ergodicity is indeed obtained from (41) by taking $E=F$ in $\mathcal{I}$. In what follows we will also need the following technical standard results.

Invariance and $\mathcal{I}$-measurability. A function $\mathbf{h}: \Sigma \rightarrow \mathbb{R}$ is $\mathcal{I}$-measurable if and only if it is invariant under the group $\left(T_{z}\right)_{z \in \mathbb{Z}}$, i.e., $\mathbf{h} \circ T_{z}=\mathbf{h}$ for all $z \in \mathbb{Z}^{N}$. For implication

( $h$ is $\mathcal{I}$-measurable $\Longrightarrow h$ is invariant),

the claim is indeed the straightforward consequence of

$$
T_{z}^{-1} \mathbf{h}^{-1}(\{\mathbf{h}(\omega)\})=h^{-1}(\{\mathbf{h}(\omega)\}) \Longleftrightarrow \mathbf{h}\left(T_{z}(\omega)\right)=\mathbf{h}(\omega) .
$$

The other implication is immediate.

The conditional Lebesgue dominated convergence theorem. Let $\left(\mathbf{h}_{n}\right)_{n \in \mathbb{N}}$ be a sequence in $L_{\mathbf{P}}(\Sigma)$ such that $\mathbf{h}_{n} \rightarrow \mathbf{h}, \mathbf{P}$-a.s. in $\Sigma$, and assume that there exists $\widetilde{\mathbf{h}} \in L_{\mathbf{P}}(\Sigma)$ such that $\left|\mathbf{h}_{n}\right| \leq \widetilde{\mathbf{h}}$ for all $n \in \mathbb{N}$. Let $\mathcal{G}$ be a sub $\sigma$-algebra of $\mathcal{A}$, then $\mathbf{E}^{\mathcal{G}} \mathbf{h}_{n} \rightarrow \mathbf{E}^{\mathcal{G}} \mathbf{h}, \mathbf{P}$-a.s. in $\Sigma$. The proof follows a similar method as in the proof of the standard Lebesgue dominated convergence theorem, using the conditional Fatou Lemma instead of the standard Fatou Lemma.

In the next two sections, $\left(\Sigma, \mathcal{A}, \mathbf{P},\left(T_{z}\right)_{z \in \mathbb{Z}^{N}}\right)$ is a given discrete dynamical system.

5.1.1. The random diffusion part. Given $\alpha>0, \beta>0$, and $\gamma>0$, we denote by $\operatorname{Conv}_{\alpha, \beta, \gamma}$ the class of functions $g: \mathbb{R}^{N} \times \mathbb{R}^{N} \rightarrow \mathbb{R},(x, \xi) \mapsto g(x, \xi)$, satisfying conditions $\left(\mathrm{D}_{1, n}\right),\left(\mathrm{D}_{2, n}\right)$, and $\left(\mathrm{D}_{3, n}\right)$, The class $\operatorname{Conv}_{\alpha, \beta, \gamma}$ is endowed with the $\sigma$-algebra $\mathcal{T}_{\operatorname{Conv}_{\alpha, \beta, \gamma}}$ which is the trace of the product $\sigma$-algebra of $\mathbb{R}^{\mathbb{R}^{N} \times \mathbb{R}^{N}}$, i.e., the smallest $\sigma$-algebra on $\operatorname{Conv}_{\alpha, \beta, \gamma}$ such that all the evaluation maps

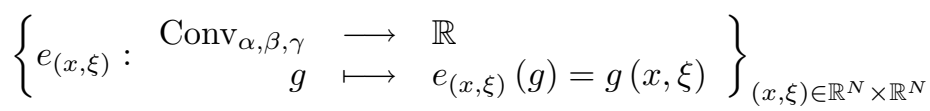

are measurable.

We consider a random convex integrand $W: \Sigma \times \mathbb{R}^{N} \times \mathbb{R}^{N} \rightarrow \mathbb{R}$, i.e., a $\left(\mathcal{A} \otimes \mathcal{B}\left(\mathbb{R}^{N}\right) \otimes \mathcal{B}\left(\mathbb{R}^{N}\right), \mathcal{B}(\mathbb{R})\right)$ measurable function such that for every $\omega \in \Sigma$, the function $W(\omega, \cdot, \cdot)$, belongs to the class $\operatorname{Conv}_{\alpha, \beta, \gamma}$. Since $W(\cdot, x, \zeta)$ is $(\mathcal{A}, \mathcal{B}(\mathbb{R}))$-measurable for all $(x, \xi) \in \mathbb{R}^{N} \times \mathbb{R}^{N}$, the map $\widetilde{W}: \Sigma \rightarrow \operatorname{Conv}_{\alpha, \beta, \gamma}$, $\omega \mapsto W(\omega, \cdot, \cdot)$, is $\left(\mathcal{A}, \mathcal{T}_{\operatorname{Conv}_{\alpha, \beta, \gamma}}\right)$-measurable, and we denote by $\widetilde{\mathbf{P}}$ its law, that is $\widetilde{\mathbf{P}}=\widetilde{W} \# \mathbf{P}$.

We assume that $W$ satisfies the following covariance property with respect to the dynamical system $\left(\Sigma, \mathcal{A}, \mathbf{P},\left(T_{z}\right)_{z \in \mathbb{Z}^{N}}\right)$ : for all $z \in \mathbb{Z}^{N}$

$$
W\left(T_{z} \omega, x, \xi\right)=W(\omega, x+z, \xi) \text { for a.e. } x \in \mathbb{R}^{N} \text {, for all } \xi \in \mathbb{R}^{N} \text {, and for } \mathbf{P} \text {-a.e. } \omega \in \Sigma \text {. }
$$

For all $g$ in $\operatorname{Conv}_{\alpha, \beta, \gamma}$ and all $z \in \mathbb{Z}^{N}$, let us set $\widetilde{T}_{z} g(x, \cdot)=g(x+z, \cdot)$. This defines a group $\left(\widetilde{T}_{z}\right)_{z \in \mathbb{Z}^{N}}$ which acts on $\operatorname{Conv}_{\alpha, \beta, \gamma}$, and clearly, $\widetilde{T}_{z}: \operatorname{Conv}_{\alpha, \beta, \gamma} \rightarrow \operatorname{Conv}_{\alpha, \beta, \gamma}$ is $\mathcal{T}_{\operatorname{Conv}_{\alpha, \beta, \gamma}}$-measurable for all $z \in \mathbb{Z}^{N}$. 
Then it is easy to show that the covariance property implies that the law $\widetilde{\mathbf{P}}$ of $\widetilde{W}$ is invariant under the $\operatorname{group}\left(\widetilde{T}_{z}\right)_{z \in \mathbb{Z}^{N}}$, that is $\widetilde{T}_{z} \# \widetilde{\mathbf{P}}=\widetilde{\mathbf{P}}$ for all $z \in \mathbb{Z}^{N}$. We say that the random function $W$ is periodic in law.

We write $\varepsilon$ to denote a sequence $\left(\varepsilon_{n}\right)_{n \in \mathbb{N}}$ of positive numbers with $\lim _{n \rightarrow+\infty} \varepsilon_{n}=0$, which is denoted by $\varepsilon \rightarrow 0$. Then, the following random functional $\widetilde{\Phi}_{\varepsilon}: \Sigma \times L^{2}(\Omega) \longrightarrow \mathbb{R}_{+} \cup\{+\infty\}$ defined by

$$
\widetilde{\Phi}_{\varepsilon}(\omega, u)=\left\{\begin{array}{cl}
\int_{\Omega} W\left(\omega, \frac{x}{\varepsilon}, \nabla u\right) d x & \text { if } u \in H^{1}(\Omega) \\
+\infty & \text { otherwise. }
\end{array}\right.
$$

models a random energy concerning various steady-states situations, where the small parameter $\varepsilon$ accounts the size of the randomly distributed heterogeneities in the context of a statistically homogeneous media. The measurability of $\omega \mapsto \widetilde{\Phi}_{\varepsilon}(\omega, u)$ for all $u \in H^{1}(\Omega)$ may be obtained by standard arguments (see for instance [8, Section 12.4.3 and Proposition 12.4.1]).

Under the hypotheses above on $\widetilde{W}$ with respect to the discrete dynamical system $\left(\Sigma, \mathcal{A}, \mathbf{P},\left(T_{z}\right)_{z \in \mathbb{Z}^{N}}\right)$, it is now standard, using the subadditive ergodic theorem ([17] or [8, Theorem 12.4.3]), that for $\mathbf{P}$-a.s. $\omega$ in $\Sigma$ the sequence $\left(\widetilde{\Phi}_{\varepsilon}(\omega, \cdot)\right)_{\varepsilon>0} \Gamma$-converges to the integral functional $\widetilde{\Phi}^{h o m}(\omega, \cdot)$, where $\widetilde{\Phi}^{\text {hom }}$ : $\Sigma \times L^{2}(\Omega) \longrightarrow \mathbb{R}_{+} \cup\{+\infty\}$ is given by

$$
\widetilde{\Phi}^{\text {hom }}(\omega, u)=\left\{\begin{array}{cl}
\int_{\Omega} W^{h o m}(\omega, \nabla u) d x & \text { if } u \in H^{1}(\Omega) \\
+\infty & \text { otherwise }
\end{array}\right.
$$

when $L^{2}(\Omega)$ is equipped with its strong convergence. Let $Y$ denote the unit cell $(0,1)^{N}$, then, for every $\xi \in \mathbb{R}^{N}$, the density $W^{\text {hom }}$ is given, for $\mathbf{P}$-a.s. $\omega \in \Sigma$, by

$$
\begin{aligned}
W^{h o m}(\omega, \xi) & =\lim _{n \rightarrow+\infty} \mathbf{E}^{\mathcal{I}} \inf \left\{\frac{1}{n^{N}} \int_{n Y} W(\omega, y, \xi+\nabla u(y)) d y: u \in H_{0}^{1}(n Y)\right\} \\
& =\inf _{n \in \mathbb{N}^{*}} \mathbf{E}^{\mathcal{I}} \inf \left\{\frac{1}{n^{N}} \int_{n Y} W(\omega, y, \xi+\nabla u(y)) d y: u \in H_{0}^{1}(n Y)\right\} .
\end{aligned}
$$

If $\left(\Sigma, \mathcal{A}, \mathbf{P},\left(T_{z}\right)_{z \in \mathbb{Z}^{N}}\right)$ is ergodic, then $W^{\text {hom }}$ is deterministic and given for $\mathbf{P}$-a.s. $\omega \in \Sigma$ by

$$
\begin{aligned}
W^{h o m}(\xi) & =\lim _{n \rightarrow+\infty} \mathbf{E} \inf \left\{\frac{1}{n^{N}} \int_{n Y} W(\omega, y, \xi+\nabla u(y)) d y: u \in H_{0}^{1}(n Y)\right\} \\
& =\inf _{n \in \mathbb{N}^{*}} \mathbf{E} \inf \left\{\frac{1}{n^{N}} \int_{n Y} W(\omega, y, \xi+\nabla u(y)) d y: u \in H_{0}^{1}(n Y)\right\} .
\end{aligned}
$$

For a proof we refer the reader to [8, Proposition 12.4.3, Theorem 12.4.7] and references therein.

Given $h \in L_{\mathcal{H}^{N-1}}^{2}(\partial \Omega)$, and $a_{0} \in L_{\mathcal{H}_{N-1}}^{\infty}(\partial \Omega), a_{0} \geq 0, a_{0} \geq \sigma$ on $\Gamma \subset \partial \Omega$ with $\mathcal{H}_{N-1}(\Gamma)>0$, for some $\sigma>0$, we consider the random functionals $\Phi_{\varepsilon}$ and $\Phi^{\text {hom }}$ defined by

$$
\Phi_{\varepsilon}(\omega, u)=\left\{\begin{array}{cl}
\widetilde{\Phi}_{\varepsilon}(\omega, u)+\frac{1}{2} \int_{\partial \Omega} a_{0} u d \mathcal{H}_{N-1}-\int_{\partial \Omega} h u d \mathcal{H}_{N-1} & \text { if } u \in H^{1}(\Omega) \\
+\infty & \text { otherwise, }
\end{array}\right.
$$

and

$$
\Phi^{h o m}(\omega, u)=\left\{\begin{array}{cl}
\widetilde{\Phi}^{h o m}(\omega, u)+\frac{1}{2} \int_{\partial \Omega} a_{0} u d \mathcal{H}_{N-1}-\int_{\partial \Omega} h u d \mathcal{H}_{N-1} & \text { if } u \in H^{1}(\Omega) \\
+\infty & \text { otherwise. }
\end{array}\right.
$$

According to Lemma 3.1 for P-a.s. $\omega \in \Sigma$, the subdifferential of $\Phi_{\varepsilon}(\omega, \cdot)$ (actually its Gâteaux derivative) is the operator $A_{\varepsilon}(\omega): L^{2}(\Omega) \rightarrow 2^{L^{2}(\Omega)}$ defined for every $\omega \in \Sigma$ by

$$
\operatorname{dom} A_{\varepsilon}(\omega)=\left\{v \in H^{1}(\Omega): \operatorname{div} D_{\xi} W\left(\omega, \frac{\dot{\varepsilon}}{\varepsilon}, \nabla v\right) \in L^{2}(\Omega), a_{0} v+D_{\xi} W\left(\omega, \frac{\dot{\varepsilon}}{\varepsilon}, \nabla v\right) \cdot \mathbf{n}=h \text { on } \partial \Omega\right\}
$$


and, for all $v \in \operatorname{dom} A_{\varepsilon}(\omega)$,

$$
A_{\varepsilon}(\omega) v=-\operatorname{div} D_{\xi} W\left(\omega, \frac{\dot{\varepsilon}}{\varepsilon}, \nabla v\right) .
$$

Similarly the subdifferential of $\Phi^{h o m}(\omega, \cdot)$ is the operator $A^{\text {hom }}(\omega): L^{2}(\Omega) \rightarrow 2^{L^{2}(\Omega)}$ defined for every $\omega \in \Sigma$ by

$$
\operatorname{dom} A^{h o m}(\omega)=\left\{v \in H^{1}(\Omega): \operatorname{div} \partial_{\xi} W^{h o m}(\omega, \nabla v) \in L^{2}(\Omega), a_{0} v+\partial_{\xi} W^{h o m}(\omega, \nabla v) \cdot \mathbf{n}=h \text { on } \partial \Omega\right\}
$$

and, for all $v \in \operatorname{dom} A(\omega)$,

$$
A^{h o m}(\omega) v=-\operatorname{div} \partial_{\xi} W^{h o m}(\omega, \nabla v) .
$$

When $W$ is ergodic, then $A^{\text {hom }}$ is deterministic and

$$
A^{h o m} v=-\operatorname{div} \partial_{\xi} W^{h o m}(\nabla v) .
$$

Note that we can not guarantee a priori that $W^{h o m}(\omega, \cdot)$ is Gâteaux-differentiable, hence $\partial_{\xi} W^{h o m}(\omega, \cdot)$ is possibly multivalued. Nevertheless, to shorten the notation, we write indifferently $\partial_{\xi} W^{\text {hom }}(\omega, \cdot)$ to denote the subdiffrential $\partial_{\xi} W^{h o m}(\omega, \cdot)$ or any of its elements. We emphasize the fact that $A^{\text {hom }}(\omega)$ is the $\mathbf{P}$-a.s. graph limit of the operator $A_{\varepsilon}(\omega)$, and that under the following additional condition on the Fenchel conjugate of $\xi \mapsto W(\omega, x, \xi)$, the density $W^{\text {hom }}(\omega, \cdot)$ is Gâteaux differentiable for $\mathbf{P}$ a.e. $\omega \in \Sigma$, so that $\partial_{\xi} W^{h o m}(\omega, \cdot)$ is univalent and reduced to a pointwise limit:

$\left(\mathrm{D}_{3}^{*}\right)$ there exists $\gamma^{*}>0$ such that $\left\langle\xi_{1}^{*}-\xi_{2}^{*}, \xi^{1}-\xi^{2}\right\rangle \geq \gamma^{*}\left|\xi_{1}-\xi_{2}\right|^{2}$ for $\mathbf{P}$ a.e. $\omega \in \Sigma$, for a.e. $x \in \mathbb{R}^{N}$, for all $\left(\xi_{1}, \xi_{2}\right) \in \mathbb{R}^{N} \times \mathbb{R}^{N}$ and all $\left(\xi_{1}^{*}, \xi_{2}^{*}\right) \in \partial_{\xi} W^{*}\left(\omega, x, \xi_{1}\right) \times \partial_{\xi} W^{*}\left(\omega, x, \xi_{2}\right)$.

For a proof, we refer the reader to [8, Proposition 17.4.6].

5.1.2. The random reaction part. We consider a regular CP-structured random functional, i.e., a functional

$$
F: \Sigma \times[0,+\infty) \times L^{2}(\Omega) \rightarrow \mathbb{R}^{\Omega}
$$

defined by $F(\omega, t, v)(x)=f(\omega, t, x, v(x))$ where

$$
f: \Sigma \times[0,+\infty) \times \mathbb{R}^{N} \times \mathbb{R} \rightarrow \mathbb{R}
$$

is a $\left(\mathcal{A} \otimes \mathcal{B}(\mathbb{R}) \otimes \mathcal{B}\left(\mathbb{R}^{N}\right) \otimes \mathcal{B}(\mathbb{R}), \mathcal{B}(\mathbb{R})\right)$-measurable function such that for $\mathbf{P}$-a.s. $\omega \in \Sigma,(t, x, \zeta) \mapsto$ $f(\omega, t, x, \zeta)$ is a regular CP-structured reaction function associated with $(r(\omega), g, q(\omega, \cdot))$. Furthermore, we make the following additional hypotheses on $r$ and $q$ : we assume that $r \in W^{1,2}\left(0, T, L_{\text {loc }}^{2}\left(\mathbb{R}^{N}, \mathbb{R}^{l}\right)\right)$, $q \in W^{1,2}\left(0, T, L_{\text {loc }}^{2}\left(\mathbb{R}^{N}\right)\right)$, that for all bounded Borel sets $B$ of $\mathbb{R}^{N}$, the real valued functions

$$
\begin{aligned}
\omega & \mapsto r(\omega, t, \cdot) \|_{L^{2}\left(B, \mathbb{R}^{l}\right)}^{2} \text { for all } t \in[0, T], \\
\omega & \mapsto q(\omega, t, \cdot) \|_{L^{2}(B)}^{2} \text { for all } t \in[0, T], \\
\omega & \mapsto \int_{0}^{T}\left\|\frac{d r}{d t}(\omega, \tau, \cdot)\right\|_{L^{2}\left(B, \mathbb{R}^{l}\right)}^{2} d \tau \\
\omega & \mapsto \int_{0}^{T}\left\|\frac{d q}{d t}(\omega, \tau, \cdot)\right\|_{L^{2}(B)}^{2} d \tau
\end{aligned}
$$

belong to $L_{\mathbf{P}}(\Sigma)$, and that $r$ and $q$, satisfy the covariance property with respect to the dynamical system $\left(\Sigma, \mathcal{A}, \mathbf{P},\left(T_{z}\right)_{z \in \mathbb{Z}^{N}}\right)$, i.e., that for all $z \in \mathbb{Z}^{N}$, all $t \in[0,+\infty)$, a.e. $x \in \mathbb{R}^{N}$ and $\mathbf{P}$-a.s. $\omega \in \Sigma$,

$$
\begin{aligned}
& r(\omega, t, x+z)=r\left(T_{z} \omega, t, x\right), \\
& q(\omega, t, x+z)=q\left(T_{z} \omega, t, x\right) .
\end{aligned}
$$

We set $f_{\varepsilon}(\omega, t, x, \zeta):=f\left(\omega, t, \frac{x}{\varepsilon}, \zeta\right)$, and define the functional $F_{\varepsilon}$ by $F_{\varepsilon}(\omega, t, v)(x)=f\left(\omega, t, \frac{x}{\varepsilon}, v(x)\right)$. Note that in the expression of the condition $(\mathrm{CP})$ the functions $\underline{f}, \bar{f}, \underline{y}, \bar{y}$, and the numbers $\underline{\rho}, \bar{\rho}$ may depend on $\omega$ (we sometimes omit it to shorten the notation), and that $F_{\varepsilon}$ is a CP-structured reaction functional whose condition (CP) is exactly that of $F$, i.e., with the functions $\underline{f}, \bar{f}, \underline{y}, \bar{y}, \underline{\rho}$ and $\bar{\rho}$. Since $y$ and $\bar{y}$ do not depend on $\varepsilon$, condition (15) is automatically satisfied. We assume that condition (16) or condition (17) is satisfied, i.e., $a_{0} \rho \leq h \leq a_{0} \bar{\rho}$ or $\rho \leq 0 \leq \bar{\rho}$. Let us show that (14) holds for P-a.s. $\omega \in \Sigma$. This condition is a straightforward consequence of the following more accurate result. 
Lemma 5.1. There exists $N_{\text {lem }}$ in $\mathcal{A}$ with $\mathbf{P}\left(N_{\text {lem }}\right)=0$ such that for all $\omega \in \Sigma \backslash N_{\text {lem }}$, we have

$$
\begin{aligned}
& \lim _{\varepsilon \rightarrow 0} \int_{0}^{T}\left\|\frac{d r}{d t}\left(\omega, \tau, \frac{\dot{s}}{\varepsilon}\right)\right\|_{L^{2}\left(\Omega, \mathbb{R}^{l}\right)}^{2} d \tau=\mathcal{L}_{N}(\Omega) \mathbf{E}^{\mathcal{I}} \int_{0}^{T}\left\|\frac{d r}{d t}(\omega, \tau, \cdot)\right\|_{L^{2}\left(Y, \mathbb{R}^{l}\right)}^{2} d \tau, \\
& \limsup _{\varepsilon \rightarrow 0} \int_{0}^{T}\left\|\frac{d r}{d t}\left(\omega, t, \frac{\cdot}{\varepsilon}\right)\right\|_{L^{2}\left(\Omega, \mathbb{R}^{l}\right)} d t \leq\left[T \mathcal{L}_{N}(\Omega) \mathbf{E}^{\mathcal{I}} \int_{0}^{T}\left\|\frac{d r}{d \tau}(\omega, \tau, \cdot)\right\|_{L^{2}\left(Y, \mathbb{R}^{l}\right)}^{2} d \tau\right]^{\frac{1}{2}}, \\
& \lim _{\varepsilon \rightarrow 0} \int_{0}^{T}\left\|\frac{d q}{d t}\left(\omega, \tau, \frac{\cdot}{\varepsilon}\right)\right\|_{L^{2}(\Omega)}^{2} d \tau=\mathcal{L}_{N}(\Omega) \mathbf{E}^{\mathcal{I}} \int_{0}^{T}\left\|\frac{d q}{d t}(\omega, \tau, \cdot)\right\|_{L^{2}(Y)}^{2} d \tau, \\
& \limsup _{\varepsilon \rightarrow 0} \int_{0}^{T}\left\|\frac{d q}{d t}\left(\omega, t, \frac{\cdot}{\varepsilon}\right)\right\|_{L^{2}(\Omega)} d t \leq\left[T \mathcal{L}_{N}(\Omega) \mathbf{E}^{\mathcal{I}} \int_{0}^{T}\left\|\frac{d q}{d t}(\omega, \tau, \cdot)\right\|_{L^{2}(Y)}^{2} d \tau\right]^{\frac{1}{2}} .
\end{aligned}
$$

Proof. We only prove 49 and $(50)$, the proof of 447$)$ and $(48)$ is similar. Consider the set function $\mathbb{A}$ from the class $\mathcal{B}_{b}\left(\mathbb{R}^{N}\right)$ of bounded Borel subsets of $\mathbb{R}^{N}$ into the space $L_{\mathbf{P}}^{1}(\Sigma)$ of $\mathbf{P}$-integrable real valued functions, defined by

$$
\mathbb{A}(B)(\cdot)=\int_{0}^{T}\left\|\frac{d q}{d \tau}(\cdot, \tau, \cdot)\right\|_{L^{2}(B)}^{2} d \tau .
$$

From 45 , the process $\mathbb{A}$ is well defined. Then, for every $(A, B) \in \mathcal{B}_{b}\left(\mathbb{R}^{N}\right) \times \mathcal{B}_{b}\left(\mathbb{R}^{N}\right)$ with $A \cap B=\emptyset$, from additivity of the integral we have

$$
\mathbb{A}(A \cup B)=\mathbb{A}(A)+\mathbb{A}(B) .
$$

Moreover, from 46 we deduce that

$$
\mathbb{A}(z+B)=\mathbb{A}(B) \circ T_{z} .
$$

Furthermore, A fulfills the following domination property: for all Borel set $A$ included in $Y$,

$$
\mathbb{A}(A) \leq h:=\int_{0}^{T}\left\|\frac{d q}{d \tau}(\cdot, \tau, \cdot)\right\|_{L^{2}(Y)}^{2} d \tau
$$

with $h \in L_{\mathbf{P}}^{1}(\Sigma)$. Therefore, $\mathbb{A}$ is an additive process indexed by $\mathcal{B}_{b}\left(\mathbb{R}^{N}\right)$, covariant with respect to $\left(T_{z}\right)_{z \in \mathbb{Z}^{N}}$ (see [8, Definition 12.4.1] and references therein). According to the additive ergodic theorem (see [8, Theorem 12.4.1]), there exists $N \in \mathcal{A}$ with $\mathbf{P}(N)=0$ such that for all $7 \omega \in \Sigma \backslash N$,

$$
\begin{aligned}
\lim _{\varepsilon \rightarrow 0} \frac{\mathbb{A}\left(\frac{1}{\varepsilon} \Omega\right)}{\mathcal{L}_{N}\left(\frac{1}{\varepsilon} \Omega\right)} & =\lim _{\varepsilon \rightarrow 0} \int_{0}^{T} \frac{\varepsilon^{N}}{\mathcal{L}_{N}(\Omega)}\left\|\frac{d q}{d \tau}(\omega, \tau, \cdot)\right\|_{L^{2}\left(\frac{1}{\varepsilon} \Omega\right)}^{2} d \tau \\
& =\mathbf{E}^{\mathcal{I}} \int_{0}^{T}\left\|\frac{d q}{d \tau}(\omega, \tau, \cdot)\right\|_{L^{2}(Y)}^{2} d \tau .
\end{aligned}
$$

Hence, a change of scale gives

$$
\lim _{\varepsilon \rightarrow 0} \int_{0}^{T}\left\|\frac{d q}{d \tau}\left(\omega, \tau, \frac{\cdot}{\varepsilon}\right)\right\|_{L^{2}(\Omega)}^{2} d \tau=\mathcal{L}_{N}(\Omega) \mathbf{E}^{\mathcal{I}} \int_{0}^{T}\left\|\frac{d q}{d \tau}(\omega, \tau, \cdot)\right\|_{L^{2}(Y)}^{2} d \tau .
$$

We obtain 50 by combining with

$$
\int_{0}^{T}\left\|\frac{d q}{d t}\left(\omega, t, \frac{\cdot}{\varepsilon}\right)\right\|_{L^{2}(\Omega)} d t \leq T^{\frac{1}{2}}\left[\int_{0}^{T}\left\|\frac{d q}{d \tau}\left(\omega, \tau, \frac{\cdot}{\varepsilon}\right)\right\|_{L^{2}(\Omega)}^{2} d \tau\right]^{\frac{1}{2}} .
$$

This completes the proof

${ }^{7}$ Strictly speaking the almost sure convergence holds when $\Omega$ is a convex set. Using approximation of $\Omega$ by finite union of convex subset, it is easy to show that the convergence holds for regular $\Omega$ of class $C^{1}$ (see [14, Remark 3.3]). 
5.2. General homogenization theorem for a class of nonlinear reaction-diffusion equations. Given a sequence $\left(u_{\varepsilon}^{0}\right)$ of $\left(\mathcal{A}, \mathcal{B}\left(L^{2}(\Omega)\right)\right)$-measurable functions $u_{\varepsilon}^{0}: \Sigma \rightarrow H^{1}(\Omega)$, by combining Theorem 4.1 of the previous section together with the variational convergence of the sequence of random energies $\Phi_{\varepsilon}$ specified above, we intend to analyze the asymptotic behavior in $C\left(0, T, L^{2}(\Omega)\right)$ of the solution $u_{\varepsilon}(\omega)$ of the random reaction-diffusion problem when $\varepsilon \rightarrow 0$ :

$$
\left(\mathcal{P}_{\varepsilon}(\omega)\right)\left\{\begin{array}{l}
\frac{d u_{\varepsilon}(\omega)}{d t}(t)-\operatorname{div} D_{\xi} W\left(\omega, \frac{\dot{-}}{\varepsilon}, \nabla u_{\varepsilon}(\omega)(t)\right)=F_{\varepsilon}\left(\omega, t, u_{\varepsilon}(\omega)(t)\right) \text { in } L^{2}(\Omega), \text { for a.e. } t \in(0, T) \\
u_{\varepsilon}(\omega, 0)=u_{\varepsilon}^{0}(\omega), \underline{\rho}(\omega) \leq u_{\varepsilon}^{0}(\omega, \cdot) \leq \bar{\rho}(\omega), \\
\left.\left.a_{0} u_{\varepsilon}(\omega)(t)+D_{\xi} W\left(\omega, \frac{\dot{-}}{\varepsilon}, \nabla u_{\varepsilon}(\omega)(t)\right) \cdot \mathbf{n}=h \text { on } \partial \Omega \text { for all } t \in\right] 0, T\right] .
\end{array}\right.
$$

Theorem 5.1. For each $\omega \in \Sigma$, let us denote by $u_{\varepsilon}(\omega)$ the unique solution in $C\left([0, T], L^{2}(\Omega)\right)$ of the (random) reaction-diffusion problem $\left(\mathcal{P}_{\varepsilon}(\omega)\right)$. Assume that for $\mathbf{P}$-a.s. $\omega \in \Sigma, u_{\varepsilon}^{0}(\omega)$ strongly converges to $u^{0}(\omega)$ in $L^{2}(\Omega)$, and that $\sup _{\varepsilon>0} \Phi_{\varepsilon}\left(\omega, u_{\varepsilon}^{0}(\omega)\right)<+\infty$. Then, for $\mathbf{P}$-a.s. $\omega \in \Sigma, u_{\varepsilon}(\omega)$ uniformly converges in $C\left([0, T], L^{2}(\Omega)\right)$ to the unique solution of the reaction-diffusion problem

$$
\left(\mathcal{P}^{\text {hom }}(\omega)\right)\left\{\begin{array}{l}
\frac{d u(\omega)}{d t}(t)-\operatorname{div} \partial_{\xi} W^{h o m}(\omega, \nabla u(\omega)(t)) \ni F^{\text {hom }}(\omega, t, u(\omega)(t)) \text { in } L^{2}(\Omega), \text { for a.e. } t \in(0, T) \\
u(\omega)(0)=u^{0}(\omega), \underline{y}(\omega, T) \leq u^{0}(\omega, \cdot) \leq \bar{y}(\omega, T) \\
\left.\left.a_{0} u(\omega)(t)+\partial_{\xi} W^{h o m}(\omega, \nabla u(\omega)(t)) \cdot \mathbf{n} \ni h \text { on } \partial \Omega \text { for all } t \in\right] 0, T\right]
\end{array}\right.
$$

where $F^{\text {hom }}$ is given by $F^{\text {hom }}(\omega, t, v)(x)=f^{\text {hom }}(\omega, t, x, v(x))$ with

$$
\begin{aligned}
f^{\text {hom }}(\omega, t, x, \zeta) & =\bar{r}(\omega, t) \cdot g(\zeta)+\bar{q}(\omega, t), \quad \bar{r}(\omega, t)=\mathbf{E}^{\mathcal{I}}\left(\int_{(0,1)^{N}} r(\omega, t, y) d y\right) \\
\text { and } \quad \bar{q}(\omega, t) & =\mathbf{E}^{\mathcal{I}}\left(\int_{(0,1)^{N}} q(\omega, t, y) d y\right) .
\end{aligned}
$$

Moreover, for $\mathbf{P}$-a.s. $\omega \in \Sigma, \frac{d u_{\varepsilon}(\omega)}{d t} \rightarrow \frac{d u(\omega)}{d t}$ weakly in $L^{2}\left(0, T, L^{2}(\Omega)\right)$ and $\underline{y}(\omega, T) \leq u(\omega) \leq \bar{y}(\omega, T)$.

When the dynamical system $\left(\Sigma, \mathcal{A}, \mathbf{P},\left(T_{z}\right)_{z \in \mathbb{Z}^{N}}\right)$ is ergodic, the initial condition is deterministic, i.e., $u_{\varepsilon}^{0}(\omega)=u_{\varepsilon}^{0}$ for $\mathbf{P}$-a.s. $\omega \in \Sigma$, together with $\underline{\rho}, \underline{f}, \bar{\rho}$, and $\bar{f}$, then $\left(\mathcal{P}^{\text {hom }}(\omega)=\mathcal{P}^{\text {hom }}\right)$ is deterministic and is given by

$$
\left(\mathcal{P}^{h o m}\right)\left\{\begin{array}{l}
\frac{d u}{d t}(t)-\operatorname{div} \partial_{\xi} W^{h o m}(\nabla u(t)) \ni F^{h o m}(t, u(t)) \text { in } L^{2}(\Omega), \text { for a.e. } t \in(0, T) \\
u(0)=u^{0}, \underline{y}(T) \leq u^{0}(\cdot) \leq \bar{y}(T), \\
\left.\left.a_{0} u(t)+\partial_{\xi} W^{h o m}(\nabla u(t)) \cdot \mathbf{n} \ni h \text { on } \partial \Omega \text { for all } t \in\right] 0, T\right]
\end{array}\right.
$$

where $F^{\text {hom }}$ is given by $F^{\text {hom }}(t, v)(x)=f^{\text {hom }}(t, x, v(x))$ with

$f^{h o m}(t, x, \zeta)=\bar{r}(t) \cdot g(\zeta)+\bar{q}(t), \bar{r}(t)=\mathbf{E}\left(\int_{(0,1)^{N}} r(\cdot, t, y) d y\right)$ and $\bar{q}(t)=\mathbf{E}\left(\int_{(0,1)^{N}} q(\cdot, t, y) d y\right)$.

Moreover, for $\mathbf{P}$-a.s. $\omega \in \Sigma, \frac{d u_{\varepsilon}(\omega)}{d t} \rightarrow \frac{d u}{d t}$ weakly in $L^{2}\left(0, T, L^{2}(\Omega)\right)$ and $\underline{y}(T) \leq u \leq \bar{y}(T)$.

If in addition $W$ satisfies $\left(\mathrm{D}_{3}^{*}\right)$, then $\partial_{\xi} W^{\text {hom }}(\omega, \nabla u(t))$ or $\partial_{\xi} W^{\text {hom }}(\nabla u(t))$ are univalent equal to $D_{\xi} W^{\text {hom }}(\omega, \nabla u(t))$ or $D_{\xi} W^{\text {hom }}(\nabla u(t))$, and the differential inclusions are equalities.

Proof. The proof is a straightforward consequence of Theorem 4.1 and consists in checking $\left(\mathrm{H}_{1}\right)$, $\left(\mathrm{H}_{5}\right)$, and $\left(\mathrm{H}_{6}\right)$. In the whole proof, we reason with the set of full probability $\Sigma^{\prime}=\Sigma \backslash N_{\text {lem }}$ where $N_{\text {lem }}$ is the $\mathbf{P}$-negligible set given by Lemma 5.1 .

Proof of $\left.\left(\mathrm{H}_{1}\right)\right]: \Phi_{\varepsilon}(\omega, \cdot) \stackrel{M}{\rightarrow} \Phi^{\text {hom }}(\omega, \cdot)$. According to [8, Theorem 12.4.7] in the scalar version, we deduce that for P-a.s. $\omega$ in $\Sigma^{\prime}$, the sequence of functional $\left(\widetilde{\Phi}_{\varepsilon}(\omega, \cdot)\right)_{\varepsilon>0}$, defined in Proposition 
4.2. $\Gamma$-converges to the random integral functional $\widetilde{\Phi}^{h o m}(\omega, \cdot)$ when $L^{2}(\Omega)$ is endowed with the strong convergence. We can conclude by using Proposition 4.2 .

Proof of $\left(\mathrm{H}_{5}\right)$ : We have to establish that for P-a.s. $\omega \in \Sigma^{\prime}, \sup _{\varepsilon>0}\|r(\omega, \cdot, \dot{\bar{\varepsilon}})\|_{L^{\infty}\left([0, T] \times \mathbb{R}^{N}, \mathbb{R}^{l}\right)}<+\infty$ and $r(\omega, \cdot, \dot{\bar{\varepsilon}}) \rightarrow \bar{r}(\omega, \cdot)$ in $L^{2}\left(0, T, L^{2}\left(\Omega, \mathbb{R}^{l}\right)\right)$.

The first claim is obvious. To show that $r(\omega, \cdot, \dot{\bar{\varepsilon}}) \rightarrow \bar{r}(\omega, \cdot)$ in $L^{2}\left(0, T, L^{2}\left(\Omega, \mathbb{R}^{l}\right)\right)$ we need the following lemma.

Lemma 5.2. There exists $N \in \mathcal{A}$ with $\mathbf{P}(N)=0$, such that for every $t \in[0, T]$ and every $\omega \in \Sigma^{\prime} \backslash N$,

$$
r\left(\omega, t, \frac{\cdot}{\varepsilon}\right) \rightarrow \bar{r}(\omega, \cdot):=\mathbf{E}^{\mathcal{I}}\left(\int_{(0,1)^{N}} r(\omega, t, y) d y\right)
$$

weakly in $L^{2}\left(\Omega, \mathbb{R}^{l}\right)$.

Proof. Fix $t \in[0, T] \cap \mathbb{Q}$. From (42) we can apply [14, Theorem 4.2]), straightforward consequence of the additive ergodic theorem (see [8, Theorem 12.4.1]): there exists $N_{t} \in \mathcal{A}$ with $\mathbf{P}\left(N_{t}\right)=0$ such that for every $\omega \in \Sigma^{\prime} \backslash N_{t}$

$$
r\left(\omega, t, \frac{\cdot}{\varepsilon}\right) \rightarrow \bar{r}(\omega, t)
$$

weakly in $L^{2}\left(\Omega, \mathbb{R}^{l}\right)$. Set $N:=\cup_{t \in[0, T] \cap \mathbb{Q}} N_{t}$. We are going to show that for all $\omega \in \Sigma^{\prime} \backslash N$, the weak convergence $r(\omega, t, \dot{\bar{\varepsilon}}) \rightarrow \bar{r}(\omega, t)$, holds for all $t \in[0, T]$. Let $\omega \in \Sigma^{\prime} \backslash N, \varphi \in L^{2}\left(\Omega, \mathbb{R}^{l}\right), t \in[0, T]$ and $\left(t_{n}\right)_{n \in \mathbb{N}}$ be a sequence in $[0, T] \cap \mathbb{Q}$ converging to $t$ with $t_{n} \leq t$. We have

$$
\left\langle r\left(\omega, t, \frac{\dot{-}}{\varepsilon}\right), \varphi\right\rangle_{L^{2}\left(\Omega, \mathbb{R}^{l}\right)}=\left\langle r\left(\omega, t_{n}, \frac{\dot{亠}}{\varepsilon}\right), \varphi\right\rangle_{L^{2}\left(\Omega, \mathbb{R}^{l}\right)}+\left\langle r\left(\omega, t, \frac{\dot{-}}{\varepsilon}\right)-r\left(\omega, t_{n}, \frac{\dot{\varepsilon}}{\varepsilon}\right), \varphi\right\rangle_{L^{2}\left(\Omega, \mathbb{R}^{l}\right)},
$$

with, from the weak convergence above, $\lim _{\varepsilon \rightarrow 0}\left\langle r\left(\omega, t_{n}, \dot{\bar{\varepsilon}}\right), \varphi\right\rangle_{L^{2}\left(\Omega, \mathbb{R}^{l}\right)}=\left\langle\bar{r}\left(\omega, t_{n}\right), \varphi\right\rangle_{L^{2}\left(\Omega, \mathbb{R}^{l}\right)}$. Let us set $R_{\varepsilon}\left(\omega, t, t_{n}\right):=\left\langle r(\omega, t, \dot{\dot{\varepsilon}})-r\left(\omega, t_{n}, \dot{\bar{\varepsilon}}\right), \varphi\right\rangle_{L^{2}\left(\Omega, \mathbb{R}^{l}\right)}$. Since $r(\omega, \cdot) \in W^{1,2}\left(0, T, L_{\mathrm{loc}}^{2}\left(\mathbb{R}^{N}, \mathbb{R}^{l}\right)\right)$, we infer that

$$
\begin{aligned}
\left|R_{\varepsilon}\left(\omega, t, t_{n}\right)\right| & \leq\left\|r\left(\omega, t, \frac{\cdot}{\varepsilon}\right)-r\left(\omega, t_{n}, \frac{\cdot}{\varepsilon}\right)\right\|_{L^{2}\left(\Omega, \mathbb{R}^{l}\right)}\|\varphi\|_{L^{2}\left(\Omega, \mathbb{R}^{l}\right)} \\
& \leq\|\varphi\|_{L^{2}\left(\Omega, \mathbb{R}^{l}\right)} \int_{t_{n}}^{t}\left\|\frac{d r}{d \tau}\left(\omega, \tau, \frac{\cdot}{\varepsilon}\right)\right\|_{L^{2}\left(\Omega, \mathbb{R}^{l}\right)} d \tau \\
& \leq\|\varphi\|_{L^{2}\left(\Omega, \mathbb{R}^{l}\right)}\left(t_{n}-t\right)^{\frac{1}{2}}\left(\int_{0}^{T}\left\|\frac{d r}{d \tau}\left(\omega, \tau, \frac{\cdot}{\varepsilon}\right)\right\|_{L^{\infty}\left(\mathbb{R}^{N}, \mathbb{R}^{l}\right)}^{2} d \tau\right)^{\frac{1}{2}} .
\end{aligned}
$$

Letting $\varepsilon \rightarrow 0$, then $n \rightarrow+\infty$ in (51), from 52 and (47) of Lemma 5.1, we deduce that

$$
\begin{aligned}
\lim _{n \rightarrow+\infty} \lim _{\varepsilon \rightarrow 0}\left\langle r\left(\omega, t, \frac{\cdot}{\varepsilon}\right), \varphi\right\rangle_{L^{2}\left(\Omega, \mathbb{R}^{l}\right)} & =\lim _{n \rightarrow+\infty}\left\langle\bar{r}\left(\omega, t_{n}\right), \varphi\right\rangle_{L^{2}\left(\Omega, \mathbb{R}^{l}\right)} \\
& =\langle\bar{r}(\omega, t), \varphi\rangle_{L^{2}\left(\Omega, \mathbb{R}^{l}\right)}
\end{aligned}
$$

which ends the proof of Lemma 5.2 provided that we justify the convergence

$$
\lim _{n \rightarrow+\infty}\left\langle\bar{r}\left(\omega, t_{n}\right), \varphi\right\rangle_{L^{2}\left(\Omega, \mathbb{R}^{l}\right)}=\langle\bar{r}(\omega, t), \varphi\rangle_{L^{2}\left(\Omega, \mathbb{R}^{l}\right)},
$$

which is a straightforward consequence of the continuity of $t \mapsto \int_{Y} r(\omega, t, y) d y$ and the conditional Lebesgue dominated convergence theorem.

Proof of $\left(\mathrm{H}_{5}\right)$ continued. Fix $\omega \in \Sigma^{\prime} \backslash N$. Let $\varphi \in L^{2}\left(0, T, L^{2}\left(\Omega, \mathbb{R}^{l}\right)\right)$. According to Lemma 5.2 . for all $t \in[0, T]$ we have

$$
\left\langle r\left(\omega, t, \frac{\cdot}{\varepsilon}\right), \varphi(t)\right\rangle_{L^{2}\left(\Omega, \mathbb{R}^{l}\right)} \rightarrow\langle\bar{r}(\omega, t), \varphi(t)\rangle_{L^{2}\left(\Omega, \mathbb{R}^{l}\right)}
$$


and the conclusion follows from the Lebesgue dominated convergence theorem. Indeed, the domination property is obtained as follows: we have

$$
\begin{aligned}
& \left|\left\langle r\left(\omega, t, \frac{\dot{ }}{\varepsilon}\right), \varphi(t)\right\rangle_{L^{2}\left(\Omega, \mathbb{R}^{l}\right)}\right| \\
& \leq\left\|r\left(\omega, t, \frac{\dot{\varepsilon}}{\varepsilon}\right)\right\|_{L^{2}\left(\Omega, \mathbb{R}^{l}\right)}\|\varphi(t)\|_{L^{2}\left(\Omega, \mathbb{R}^{l}\right)} \\
& \leq\left(\left\|r\left(\omega, 0, \frac{\dot{\varepsilon}}{\varepsilon}\right)\right\|_{L^{2}\left(\Omega, \mathbb{R}^{l}\right)}+\int_{0}^{T}\left\|\frac{d r}{d t}\left(\omega, \tau, \frac{\dot{\varepsilon}}{\varepsilon}\right)\right\|_{L^{2}\left(\Omega, \mathbb{R}^{l}\right)} d \tau\right)\|\varphi(t)\|_{L^{2}\left(\Omega, \mathbb{R}^{l}\right)} \\
& \leq \sup _{\varepsilon>0}\left(\left\|r\left(\omega, 0, \frac{\cdot}{\varepsilon}\right)\right\|_{L^{2}\left(\Omega, \mathbb{R}^{l}\right)}+\int_{0}^{T}\left\|\frac{d r}{d t}\left(\omega, \tau, \frac{\cdot}{\varepsilon}\right)\right\|_{L^{2}\left(\Omega, \mathbb{R}^{l}\right)} d \tau\right)\|\varphi(t)\|_{L^{2}\left(\Omega, \mathbb{R}^{l}\right)}
\end{aligned}
$$

where

and, from 48 ,

$$
\sup _{\varepsilon>0}\left\|r\left(\omega, 0, \frac{\dot{ }}{\varepsilon}\right)\right\|_{L^{2}\left(\Omega, \mathbb{R}^{l}\right)} \leq \mathcal{L}_{N}(\Omega)^{\frac{1}{2}}\|r(\omega, \cdot, \cdot)\|_{L^{\infty}\left([0, T] \times \mathbb{R}^{N}, \mathbb{R}^{l}\right)}
$$

$$
\sup _{\varepsilon>0} \int_{0}^{T}\left\|\frac{d r}{d t}\left(\omega, \tau, \frac{\cdot}{\varepsilon}\right)\right\|_{L^{2}\left(\Omega, \mathbb{R}^{l}\right)} d \tau<+\infty .
$$

Proof of $\left(\mathrm{H}_{6}\right)$. First, we have to prove that for P-a.s. $\omega \in \Sigma^{\prime}$, and for all $t \in[0, T]$

$$
\sup _{\varepsilon>0}\left\|q\left(\omega, t, \frac{\cdot}{\varepsilon}\right)\right\|_{L^{2}(\Omega)}<+\infty .
$$

For this, by reproducing the proof of Lemma 5.1, and using this time the additive ergodic theorem for the process $B \mapsto\|q(\omega, 0, \cdot)\|_{L^{2}(B)}^{2}$, which is well defined according to $(43)$, we easily obtain that there exists $N_{0}$ with $\mathbf{P}\left(N_{0}\right)=0$ such that for all $\omega \in \Sigma^{\prime} \backslash N_{0}$,

$$
\lim _{\varepsilon \rightarrow 0}\left\|q\left(\omega, 0, \frac{\dot{-}}{\varepsilon}\right)\right\|_{L^{2}(\Omega)}^{2}=\mathcal{L}_{N}(\Omega) \mathbf{E}^{\mathcal{I}}\|q(\omega, 0, \cdot)\|_{L^{2}(Y)}^{2} .
$$

Then for all $\omega \in \Sigma^{\prime} \backslash N_{0}$ and all $t \in[0, T]$ we have

$$
\left\|q\left(\omega, t, \frac{\dot{-}}{\varepsilon}\right)\right\|_{L^{2}(\Omega)} \leq\left\|q\left(\omega, 0, \frac{\dot{-}}{\varepsilon}\right)\right\|_{L^{2}(\Omega)}+\int_{0}^{T}\left\|\frac{d q}{d t}\left(\omega, \tau, \frac{\dot{\varepsilon}}{\varepsilon}\right)\right\|_{L^{2}(\Omega)} d \tau
$$

and the claim follows from $(53)$ and $(50)$. The rest of the proof concerning the weak convergence is exactly that of condition $\left(\mathrm{H}_{5}\right)$

\section{Appendix A. Examples of CP-Structured ReACtion FunCtionals}

Examples A.1. Let us examine a first class of examples of CP-structured reaction functionals for which condition $(\mathrm{CP})$ is readily checked. Assume that for a.e. $(t, x) \in(0,+\infty) \times \mathbb{R}, f(t, x, 0) \geq 0$ and that there exists $\rho>0$ such that $f(t, x, \rho) \leq 0$. Then $(\mathrm{CP})$ is satisfied. Indeed, take $\underline{f}=\bar{f}=0$ and $\rho=0$, $\bar{\rho}=\rho$. Then $\underline{y}=0$ and $\bar{y}=\rho$ are solution of $\underline{\mathrm{ODE}_{\mathrm{DE}}}$ and $\overline{\mathrm{ODE}}$ respectively, and

$$
\begin{aligned}
& f(t, \underline{y}(t))=0 \leq f(t, x, 0)=f(t, x, y(t)) \\
& f(t, x, \rho)=f(t, x, \bar{y}(t)) \leq 0=\bar{f}(t, \bar{y}(t)) .
\end{aligned}
$$

For various discussions and references about examples $\boldsymbol{c}$, d), e $]$ and $f$ ) below, we refer the reader to [21].

a) Example derived from food limited population models.

The Fisher logistic growth model. The reaction function is

$$
f(t, x, \zeta)=r(t, x) \zeta\left(1-\frac{\zeta}{K}\right)
$$

where $r(t, x) \geq 0$ and $K>0$. The function $g$ defined by $g(\zeta)=\zeta\left(1-\frac{\zeta}{K}\right)$ is locally Lipschitz continuous. Moreover, $f(t, x, 0)=0$ and $f(t, x, \rho) \leq 0$ for $\rho \geq K$. Therefore the functional $F$ is a $\mathrm{CP}$-structured reaction functional associated with $(r, g, 0)$.

The interpretation of this model is the following:

- $u(t, x)$ is the population density of some species at time $t$ located at $x$, 
- $r(t, x)$ is the growth rate of the population at time $t$, located at $x$,

- $K$ is the carrying capacity, i.e., the capacity of the environment to sustain the population,

- $\frac{1}{u} \frac{d u}{d t}$ is the per-capita growth rate.

The same conclusion holds for the following extension of the previous logistic function proposed by Turner-Bradley-Kirk

$$
f(t, x, \zeta)=r(t, x) \zeta^{1+\beta(1-\gamma)}\left(1-\left(\frac{\zeta}{K}\right)^{\beta}\right)^{\gamma}
$$

where $\beta>0, \gamma>0$ and $\gamma<1+\frac{1}{\beta}$ (this last condition ensures that the maximal growth is obtained for $\zeta>0$ ). For the analysis of this function and various logistic growth models, we refer the reader to 25 .

The logistic growth model with immigration (or stocking). The reaction function is

$$
f(t, x, \zeta)=r(t, x) \zeta\left(1-\frac{\zeta}{K}\right)+q(t, x)
$$

The interpretation is that of the logistic growth model where in addition $q(t, x) \geq 0$ denotes the immigration rate. We have $f(t, x, 0) \geq 0$. Assuming that $S:=\sup _{(t, x) \in[0,+\infty) \times \mathbb{R}^{N}} \frac{q}{r}(t, x)<+\infty$, we see that $f(t, x, \rho) \leq 0$ for $\rho \geq K \frac{1+\sqrt{1+\frac{4 S}{K}}}{2}$.

We will consider the logistic growth model with emigration (or harvesting) in Example A.4 because it does not fall into this category.

The Fisher logistic growth model with Allee effect. The reaction function is

$$
f(t, x, \zeta)=r(t, x) \zeta\left(1-\frac{\zeta}{K}\right)\left(\frac{\zeta-a(t, x)}{K}\right)
$$

where $0 \leq a \leq K$. The function $f$ may be written

$$
f(t, x, \zeta)=r(t, x) \frac{\zeta^{2}}{K}\left(1-\frac{\zeta}{K}\right)-r(t, x) a(t, x) \frac{\zeta}{K}\left(1-\frac{\zeta}{K}\right)
$$

and $f(t, x, 0)=0, f(t, x, \rho) \leq 0$ for $\rho \geq K$. Therefore the functional $F$ is a CP-structured reaction functional associated with $\left(\left(r_{i}, g_{i}\right)_{i=1,2}, 0\right)$ where $g_{1}(\zeta)=\frac{\zeta^{2}}{K}\left(1-\frac{\zeta}{K}\right), g_{2}(\zeta)=\frac{\zeta}{K}\left(1-\frac{\zeta}{K}\right)$ and $r_{1}=r, r_{2}=-r a$.

The interpretation of this model is the same as the one of Fisher model with the additional critical density $a$ below which the per-capita growth rate turns negative. We can also consider the logistic growth model with Allee effect and immigration by setting

$$
f(t, x, \zeta)=r(t, x) \zeta\left(1-\frac{\zeta}{K}\right)\left(\frac{\zeta-a(t, x)}{K}\right)+q(t, x)
$$

with the stocking rate $q(t, x) \geq 0$. We have $f(t, x, 0) \geq 0$ and $f(t, x, \rho) \leq 0$ for $\rho$ large enough depending on $\sup _{(t, x) \in[0,+\infty) \times \mathbb{R}^{N}} \frac{q}{r}(t, x)$.

b) Example derived from haematopoiesis (Wazewska-Czyziewska E Lasota model). The reaction function is

$$
f(t, x, \zeta)=-\mu(t, x) \zeta+P(t, x) \exp (-\gamma \zeta)
$$

with $\mu>0, P>0$, and $\gamma>0$. In this example $g_{1}(\zeta)=\zeta, g_{2}(\zeta)=\exp (-\gamma \zeta), r_{1}=-\mu$ and $r_{2}=P$. Moreover, $f(t, x, 0)=0$ and $f(t, x, \rho) \leq 0$ for $\rho \geq \sup _{(t, x) \in[0,+\infty) \times \mathbb{R}^{N}} \frac{P(t, x)}{\mu(t, x)}$ which is assumed to be finite. Therefore the functional $F$ is a CP-structured reaction functional associated with $\left(\left(r_{i}, g_{i}\right)_{i=1,2}, 0\right)$.

The interpretation of this the model is the following:

- $u(t, x)$ is the number of red-blood cell at time $t$ located at $x$,

- $\mu(t, x)$ is the probability of death of red-blood cells, $P$ and $\gamma$ are two coefficients related to the production of red-blood cells per unit time. 
For a generalization of this function in the context of delay ordinary differential equations, we refer the reader to 24 .

c) Example derived from nuclear reactor dynamics and heat conduction

First model. The reaction function is

$$
f(t, x, \zeta)=r(t, x) \zeta(a-b \zeta)+q(x)
$$

with $a>0, b>0$, and $q \geq 0$. In this example $i=1, g_{1}(\zeta)=\zeta(a-b \zeta), r_{1}=r$. Moreover, $f(t, x, 0)=q(t, x) \geq 0$ and $f(t, x, \rho) \leq 0$ for

$$
\rho \geq \frac{a+\sqrt{a^{2}+4 \overline{\left(\frac{q}{r}\right)}}}{2 b}
$$

where $\overline{\left(\frac{q}{r}\right)}=\sup _{(t, x) \in[0,+\infty) \times \mathbb{R}^{N}} \frac{q(t, x)}{r(t, x)}$ which is assumed to be finite. Therefore the functional $F$ is a CP-structured reaction functional associated with $\left(\left(r_{1}, g_{1}\right), q\right)$.

The interpretation of this the model is the following:

- $u(t, x)$ is the one velocity neutron flux at time $t$ located at $x$, i.e., the total path length covered by all neutrons in one cubic centimeter during one second, of the beam of neutrons traveling in a single direction. Mathematically, $u(t, x)=m(t, x) v(t, x)$ where $m(t, x)$ is the neutron density (neutrons $\left./ \mathrm{cm}^{3}\right)$ and $v(t, x)$ the neutron velocity $(\mathrm{cm} / \mathrm{sec})$.

- $q(t, x)$ is an additional source.

d) Example derived from heat transfer: the Stefan's-Boltzmann fourth-power law in heat transfer. The reaction function is

$$
f(t, x, \zeta)=r(t, x)\left(a^{4}-\zeta^{4}\right)
$$

with $a>0$ and $r>0$. In this example $i=1, g_{1}(\zeta)=a^{4}-\zeta^{4}, r_{1}=r$. We have $f(t, x, 0)=$ $r(t, x) a^{4}>0$ and $f(t, x, \rho) \leq 0$ for $\rho \geq a$.

The interpretation of this the model is the following:

- $T$ is temperature radiated by a black body,

- $a$ is the temperature of surroundings,

- $r$ is related to the radiating area and the emissivity of the radiator.

e) Example derived from chemical reactor and combustion models. The reaction function is

$$
f(t, x, \zeta)=-r(t, x) \zeta^{p}
$$

with $p \geq 1$ and $r(t, x) \geq 0$, or its generalization $f(t, x, \zeta)=-\left[r_{1}(t, x) \zeta^{p_{1}}+r_{2}(t, x) \zeta^{p_{2}}\right]$ with $p_{1} \geq 1$, $p_{2} \geq 1$, and $r_{1}(t, x) \geq 0, r_{2}(t, x) \geq 0$. In this example, $i=1,2, g_{i}(\zeta)=\zeta^{p_{i}}$. We have $f(t, x, 0)=0$, and $f(t, x, \rho) \leq 0$ for any $\rho>0$.

The interpretation of this the model is the following for $i=1$ :

- $u(t, x)$ is the mass concentration of the combustible material at time $t$, located at $x$ in a nonisothermal reaction,

- $r$ is given according to Arrhenius kinetics by $r(t, x)=\exp \left(\gamma-\frac{\gamma}{v(t, x)}\right)$ where $v(t, x)$ is the temperature and $\gamma$ is the Arrhenius number, and $p$ is the order of the reaction.

f) Example derived from enzyme kinetics models in biochemical system. The reaction function is

$$
f(t, x, \zeta)=-r(t, x) \frac{\zeta}{1+a \zeta}, \quad \text { or } \quad f(t, x, \zeta)=-r(t, x) \frac{\zeta}{1+a \zeta+b \zeta^{2}},
$$

with $a>0, b>0$, and $r \geq 0$. In this example, $i=1, g_{1}(\zeta)=\frac{\zeta}{1+a \zeta}$ or $\frac{\zeta}{1+a \zeta+b \zeta^{2}}$ that we extend by 0 for $\zeta<0$. We have $f(t, x, 0)=0$ and $f(t, x, \rho) \leq 0$ for any $\rho>0$.

The interpretation of this the model is the following for $i=1$ :

- $u(t, x)$ is the substrate concentration,

- $r$ depends on the total amount of enzyme and various rates of the reaction,

- $r$ depends on various rates of the reaction. 
Examples A.2. We examine now a second class of examples. We assume, as in the previous examples, that for a.e. $(t, x) \in(0,+\infty) \times \mathbb{R}, f(t, x, 0) \geq 0$, but the second condition is no longer satisfied. Nevertheless we assume that there exists a constant $M \geq 0$ such that $f \leq M$. Then $[\mathrm{CP})$ is satisfied. Indeed, take $f=0, \rho=0$ as for the previous class of examples, and $\bar{f}=M$ and $\bar{\rho}$ any positive $\rho$. Then $\underline{y}=0$ and $\bar{y}=M t+\rho$ are solution of $\underline{\mathrm{ODE}}$ and $\overline{\mathrm{ODE}}$ respectively, with

$$
\begin{aligned}
& f(t, \underline{y}(t))=0 \leq f(t, x, 0)=f(t, x, \underline{y}(t)) \\
& f(t, x, M t+\rho)=f(t, x, \bar{y}(t)) \leq M=\bar{f}(t, \bar{y}(t)) .
\end{aligned}
$$

Example derived from thermal explosions in the theory of combustion. The reaction function is

$$
f(t, x, \zeta)=\left\{\begin{array}{cc}
r(t, x) \exp \left(\gamma\left(1-\frac{1}{\zeta}\right)\right) & \text { if } \zeta>0 \\
0 & \text { if } \zeta \leq 0
\end{array}\right.
$$

In this example $i=1, r_{1}=r$ and $g_{1}=f / r$. We have $f(t, x, 0)=0$ and $f \leq M$ with $M=$ $\sup _{(t, x) \in[0,+\infty) \times \mathbb{R}^{N}} r(t, x) \exp (\gamma)$ where $\sup _{(t, x) \in[0,+\infty) \times \mathbb{R}^{N}} r(t, x)$ is assumed to be finite.

The interpretation of this model is the following:

- $u(t, x)$ is temperature at time $t$ located at $x$ in thermal explosion,

- $\gamma$ and $r$ are physical coefficients (see 21] and references therein).

Examples A.3. We deal with a third class of examples where we still assume that for a.e. $(t, x) \in$ $(0,+\infty) \times \mathbb{R}, f(t, x, 0) \geq 0$, but $f$ does not satisfies the second condition fulfilled by the two previous class of examples, but satisfies $f(t, x, \zeta) \leq a \zeta^{p}$ for some $a>0$ and $p \geq 1$. Then (CP) is satisfied. Indeed, take $f=0, \rho=0$ as for the previous class of examples, $\bar{f}(t, \zeta)=a \zeta^{p}$ and $\bar{\rho}$ any positive $\rho$. Then $y=0$ is solution of $\underline{\text { ODE }}$ and $\bar{y}$ defined by

$$
\bar{y}(t)=\left\{\begin{array}{cc}
\rho \exp (a t) & \text { when } p=1 \\
\left((1-\rho) a t+\rho^{1-p}\right)^{\frac{1}{1-p}} & \text { when } p>1
\end{array}\right.
$$

is solution of $\overline{\mathrm{ODE}}$ (when $p>1, \overline{\mathrm{ODE}}$ is the classical Bernouilli o.d.e $y^{\prime}=a y^{p}$ ) with

$$
\begin{aligned}
& f(t, \underline{y}(t))=0 \leq f(t, x, 0)=f(t, x, \underline{y}(t)) \\
& f(t, x, \bar{y}(t)) \leq a \bar{y}(t)^{p}=\bar{f}(t, \bar{y}(t)) .
\end{aligned}
$$

Example derived from nuclear reactor dynamics and heat conduction or from chemical reactor. The reaction function is

$$
f(t, x, \zeta)=r(t, x) \zeta^{p}
$$

where $p \geq 1$. In this example $i=1, r_{1}=r$ and $g_{1}=\zeta^{p}$. We have $f(t, x, 0)=0$ and $f \leq \bar{r} \zeta^{p}$, where $\bar{r}=\sup _{(t, x) \in[0,+\infty) \times \mathbb{R}^{N}} r(t, x)$ is assumed to be finite.

The interpretation of this the model is the following:

- $u(t, x)$ represents the one velocity neutron flux at time $t$ located at $x$ in case there is a positive temperature feedback. A second interpretation occurs in the scope of chemical reactor, where $u(t, x)$, this time, is the concentration of a chemical labile species (see 21] and references therein). For the case $p=2$ see 23 .

Examples A.4. We finally complete our examples by examining a last class for which the first condition $f(t, x, 0) \geq 0$ is not satisfied. We assume that there exists $\rho>0$ such that for all $(t, x) \in[0,+\infty) \times \mathbb{R}^{N}$, $f(t, x, \rho) \leq 0$ and $f(t, x, \zeta) \geq \zeta(1-\zeta)-a$ for some $a>\frac{1}{4}$. Since $\zeta(1-\zeta)-a<0$, it is not assured that $f(t, x, 0) \geq 0$. Nevertheless we claim that condition $(\mathrm{CP})$ is satisfied. Indeed take $\bar{\rho}=\rho, \bar{f}=0$ as in Examples A.1. On the other hand, take $\underline{f}(t, \zeta)=\zeta(1-\zeta)-a$ and $\underline{\rho}$ any negative number. Then $\underline{y}$ is the solution to the ordinary differential equation

and is given by

$$
\underline{\mathrm{ODE}}\left\{\begin{array}{l}
\underline{y^{\prime}}=\underline{y}(1-\underline{y})-a \\
\underline{y}(0)=\underline{\rho},
\end{array}\right.
$$

$$
\underline{y}(t)=\frac{\underline{\rho}-\frac{1-2 \underline{\rho}-\lambda^{2}}{2 \lambda} \tan \left(\frac{\lambda t}{2}\right)}{1-\frac{1-2 \underline{\rho}}{\lambda} \tan \left(\frac{\lambda t}{2}\right)},
$$


where $\lambda=\sqrt{4 a-1}$.

Example derived from food limited population models with emigration (or harvesting, or extraction). The reaction function is

$$
f(t, x, \zeta)=r(t, x) \zeta\left(1-\frac{\zeta}{K}\right)-q(t, x) .
$$

The interpretation is that of the logistic growth model where in addition $0 \leq q(t, x)$ denotes the emigration rate. The change of variable $\frac{\zeta}{K}=s$, and the change of function $\tilde{f}(t, x, s)=\frac{1}{r K} f(t, x, K s)$ where $\underline{r}(t, x)=\inf _{(t, x) \in[0,+\infty) \times \mathbb{R}^{N}} r(t, x)$ is assume to be positive, leads to $\tilde{f}(t, x, s) \geq s(1-s)-$ $\sup _{(t, x) \in[0,+\infty) \times \mathbb{R}^{N}} q(t, x)$. We are in the general situation described above provided that we assume $a:=\sup _{(t, x) \in[0,+\infty) \times \mathbb{R}^{N}} q(t, x)>\frac{\underline{r} K}{4}$. For further examples on logistic growth models with migration, we refer the reader to 9 .

\section{Appendix B. Examples of Stochastic homogenization of A DifFusive Fisher Food Limited POPUlation MODEL With Allee EFFECT}

As an application of Theorem 5.1, we treat the stochastic homogenization of the reaction-diffusion problem describing the food limited population model whose reaction function corresponds to that of the Fisher model with Allee effect (see Examples A.1 of the appendix A). We assume that the growth rate $r$, along with the critical threshold $a$ below which the per-capita growth rate turns negative, are influenced by the heterogeneities of the spatial environment and change in each small habitats. However we assume that the carrying capacity $K$ is constant. In a first example, we assume that the distribution of the heterogeneities is following a regular random patch model, i.e., in the probabilistic setting, the dynamical system is that of a random checkerboard-like environment. In a second example, the heterogeneities are distributed following a Poisson point process. In the two examples, in order to simplify the model, we assume that $r$ and $a$ do not depend on the time variable $t$. Otherwise, it would be sufficient to make the appropriate assumptions concerning the absolute continuity on $r$ and $a$ with respect to the time variable, without changing the constructions below. It is interesting to note that the homogenized critical density $a^{\text {hom }}$ is now a function of the growth rate. To shorten the notation we assume that the Fenchel conjugate of $\xi \mapsto W(\omega, x, \xi)$ satisfies $\left(\mathrm{D}_{3}^{*}\right)$

B.1. Random checkerboard-like environment. Given two triples $\left(r^{-}, a^{-}, W^{-}\right)$and $\left(r^{+}, a^{+}, W^{+}\right)$ in $[0,+\infty] \times[0, K] \times \operatorname{Conv}_{\alpha, \beta, \gamma}$ where $W^{-}, W^{+}$do not depend on $x$, and $\mathbf{p} \in[0,1]$, we consider the product $\Sigma=\left\{\left(r^{-}, a^{-}, W^{-}\right),\left(r^{+}, a^{+}, W^{+}\right)\right\}^{\mathbb{Z}^{N}}$ equipped with the $\sigma$-algebra $\mathcal{A}$, product of the trivial $\sigma$-algebra of subsets of $\left\{\left(r^{-}, a^{-}, W^{-}\right),\left(r^{+}, a^{+}, W^{+}\right)\right\}$. Each element of $\Sigma$ is then of the form $\left(\omega_{z}\right)_{z \in \mathbb{Z}^{N}}$, with $\omega_{z}=\left(\omega_{z}^{1}, \omega_{z}^{2}, \omega_{z}^{3}\right)$, where $\omega_{z}^{1} \in\left\{r^{-}, r^{+}\right\}, \omega_{z}^{2} \in\left\{a^{-}, a^{+}\right\}$, and $\omega_{z}^{3} \in\left\{W^{-}, W^{+}\right\}$.

We equip $(\Sigma, \mathcal{A})$ with the product probability measure $\mathbf{P}_{\mathbf{p}}=\otimes_{z \in \mathbb{Z}^{N}} \mu_{z}$ where $\mu_{z}=\mathbf{p} \delta_{\left(r^{-}, a^{-}, W^{-}\right)}+$ $(1-\mathbf{p}) \delta_{\left(r^{+}, a^{+}, W^{+}\right)}$for all $z \in \mathbb{Z}^{N}$. By construction $\mathbf{P}_{\mathbf{p}}$ is invariant under the shift group $\left(T_{z}\right)_{z \in \mathbb{Z}^{N}}$ defined by $T_{z}\left(\omega_{t}\right)_{t \in \mathbb{Z}^{N}}=\left(\omega_{t+z}\right)_{t \in \mathbb{Z}^{N}}$, i.e., $T_{z} \# \mathbf{P}_{\mathbf{p}}=\mathbf{P}_{\mathbf{p}}$ for all $z \in \mathbb{Z}^{N}$. We set

$$
r(\omega, x):=\omega_{z}^{1} \quad \text { and } \quad a(\omega, x)=\omega_{z}^{2}, \quad W(\omega, x, \cdot)=\omega_{z}^{3} \text { whenever } x \in Y+z,
$$

and $f(\omega, t, x, \zeta)=r(\omega, x) \zeta\left(1-\frac{\zeta}{K}\right)\left(\frac{\zeta-a(\omega, x)}{K}\right)$ which define a random CP-structured reaction function provided that we write it

$$
f(\omega, t, x, \zeta)=r(\omega, x) \frac{\zeta^{2}}{K}\left(1-\frac{\zeta}{K}\right)-r(\omega, x) a(\omega, x) \frac{\zeta}{K}\left(1-\frac{\zeta}{K}\right) .
$$

According to this definition it is straightforward to show that $f$ is a random CP-structured reaction function, that $f(\omega, t, x+z, \cdot)=f\left(T_{z} \omega, t, x, \xi\right)$ for all $(\omega, x, \xi) \in \Sigma \times \mathbb{R}^{N} \times \mathbb{R}^{N}$, and that conditions 42 and (43) hold. Regarding the random density $W$, one can easily show that it verify $W(\omega, x+z, \xi)=$ $W\left(T_{z} \omega, x, \xi\right)$.

Furthermore, it is easily seen that $\left(\Sigma, \mathcal{A}, \mathbf{P}_{\mathbf{p}},\left(T_{z}\right)_{z \in \mathbb{Z}^{N}}\right)$ is ergodic since its satisfies the mixing condition (41) (notice that 41 is satisfied with the cylinders which generate $\mathcal{A}$ ).

The random CP-structured reaction function $f_{\varepsilon}$ defined by $f_{\varepsilon}(\omega, t, x, \zeta)=f\left(\omega, t, \frac{x}{\varepsilon}, \zeta\right)$ may be seen as the Fisher reaction function defined in a checkerboard-like spatial environment, i.e., the growth rate $r$ and 
the threshold $a$ take two values at random on the lattice spanned by the unit cell $Y=(0,1)^{2}$ modeling a mosaic of two kinds of small habitats. The diffusion is associated with a random density $W_{\varepsilon}$ defined by $W_{\varepsilon}(\omega, x, \xi)=W\left(\omega, \frac{x}{\varepsilon}, \xi\right)$ taking also two values at random on this lattice. The triples $\left(r^{-}, a^{-}, W^{-}\right)$and $\left(r^{+}, a^{+}, W^{+}\right)$represent a sample of two kinds of habitat whose probability of occurring is $\mathbf{p}$ and $1-\mathbf{p}$ respectively. Obviously, we can easily generalize this model with $r$, $a$ and $W$ taking countable values. To shorten the notation, we assume that $W^{ \pm}$satisfy $\left(\mathrm{D}_{3}^{*}\right)$ so that $W^{\text {hom }}$ is Gâteaux differentiable, and is reduced to a pointwise limit.

The reaction-diffusion problem modeling the evolution of the density $u$ of some species during a time $T>0$, in a $C^{1}$-regular domain $\Omega$ included in a random checkerboard-like environment, when no species is located on the boundary, and when the density $u_{\varepsilon}^{0}$ at time $t=0$ is regular and known, is given by

$$
\left\{\begin{array}{l}
\frac{d u_{\varepsilon}}{d t}(\omega, t)-\operatorname{div} D_{\xi} W\left(\omega, \frac{\dot{-}}{\varepsilon}, \nabla u_{\varepsilon}(\omega, t)\right)= \\
r\left(\omega, \frac{\dot{-}}{\varepsilon}\right) u_{\varepsilon}(\omega, t)\left(1-\frac{u_{\varepsilon}(\omega, t)}{K_{c a r}}\right)\left(\frac{u_{\varepsilon}(\omega, t)-a(\omega, \dot{\bar{\varepsilon}})}{K_{\text {car }}}\right) \text { a.e. in }(0, T) \times \Omega \\
u_{\varepsilon}(\omega, 0)=u_{\varepsilon}^{0}, 0 \leq u_{\varepsilon}^{0} \leq K_{\text {car }}, \\
\left.\left.u_{\varepsilon}(\omega, t) \in H^{1}(\Omega), \operatorname{div} D_{\xi} W\left(\omega, \dot{\bar{\varepsilon}}, \nabla u_{\varepsilon}(\omega, t)\right) \in L^{2}(\Omega) \text { for all } t \in\right] 0, T\right], \\
\left.\left.u_{\varepsilon}(\omega, t)=0 \text { on } \partial \Omega \text { for all } t \in\right] 0, T\right] .
\end{array}\right.
$$

We assume that the initial density $u_{\varepsilon}^{0}$ strongly converges to some $u_{0}$ in $L^{2}(\Omega)$. According to Theorem 5.1. we can say that when $\varepsilon$ is very small compared to the size of the domain $\Omega$, a deterministic model, well aware with the evolution species, is given by

$$
\left\{\begin{array}{l}
\frac{d u}{d t}(t)-\operatorname{div} D_{\xi} W^{h o m}(\nabla u(t))=\bar{r} u(t)\left(1-\frac{u(t)}{K_{c a r}}\right)\left(\frac{u(t)-\frac{\overline{r a}}{\bar{r}}}{K_{\text {car }}}\right) \text { for a.e. }(t, x) \in(0, T) \times \Omega \\
u(0)=u_{0}, 0 \leq u^{0} \leq K_{\text {car }}, \\
u(t) \in H^{1}(\Omega), \operatorname{div}\left(D_{\xi} W^{h o m}(\nabla u(t))\right) \in L^{2}(\Omega), \\
u(t)=0 \text { on } \partial \Omega \text { for all } t \in] 0, T] .
\end{array}\right.
$$

where

$$
\begin{aligned}
& W^{\text {hom }}(\xi)=\inf _{n \in \mathbb{N}^{*}} \mathbf{E} \text { inf }\left\{\frac{1}{n^{2}} \int_{n Y} W(\omega, y, \xi+\nabla u(y)) d y: u \in H_{0}^{1}(Y)\right\} \\
& \bar{r}=\mathbf{E}\left(\int_{Y} r(\omega, y) d y\right)=\mathbf{p} r^{-}+(1-\mathbf{p}) r^{+}, \overline{r a}=\mathbf{E}\left(\int_{Y} r(\omega, y) a(\omega, y) d y\right)=\mathbf{p} r^{-} a^{-}+(1-\mathbf{p}) r^{+} a^{+}
\end{aligned}
$$

Everything happens as if the density evolution of the species took place in a homogeneous environment following a Fisher diffusive model with Allee effect and constant coefficients. Concerning the solution $u$, it is interesting to note that the growth rate is deterministic and constant in the environment, and that the critical density $a^{\text {hom }}=\frac{\overline{r a}}{\bar{r}}$ which still satisfies $0 \leq a^{h o m} \leq K$, is now a function of the growth rate and is a monotone function of the probability $\mathbf{p}$. The diffusion operator is now governed by an homogeneous and deterministic operator obtained as an almost sure graph limit.

B.2. Environment whose heterogeneities are independently randomly distributed with a frequency $\lambda$. As a first step, we are going to define a discrete dynamical system $\left(\Sigma, \mathcal{A}, \mathbf{P}_{\lambda},\left(T_{z}\right)_{z \in \mathbb{Z}^{N}}\right)$, modeling the environment whose heterogeneities are spheres and whose centers are randomly distributed with a frequency $\lambda$ per unit area. We assume that the number of centers is locally finite and that the numbers of centers in two disjointed regions are two independent random variables. The growth rate and the threshold in the Fisher reaction function with Allee effect, together with the density associated with the random diffusion, must take different values outside or inside the heterogeneities.

Denote by $\mathcal{M}$ the set of countable and locally finite sums of Dirac measures in $\mathbb{R}^{2}$, equipped with the $\sigma$-algebra generated by all the evaluation maps $\mathcal{E}_{B}: m \mapsto m(B)$ from $\mathcal{M}$ into $\mathbb{N} \cup\{+\infty\}$ when $B$ belongs to $\mathcal{B}\left(\mathbb{R}^{2}\right)$. Then, given $\lambda>0$, there exists a subset $\Sigma$ of locally finite sequences $\left(\omega_{i}\right)_{i \in \mathbb{N}}$ in $\mathbb{R}^{2}$, a 
probability space $\left(\Sigma, \mathcal{A}, \mathbf{P}_{\lambda}\right)$ and a point process, called Poisson point process, $\mathcal{N}: \omega \mapsto \mathcal{N}(\omega, \cdot)$ from $\Sigma$ into $\mathcal{M}$ satisfying

(i) $\mathcal{N}(\omega, \cdot)=\sum_{i \in \mathbb{N}} \delta_{\omega_{i}}$, where we identify the sequence $\left(\omega_{i}\right)_{i \in I}$ with the set $\left\{\omega_{i}: i \in \mathbb{N}\right\}$;

(ii) for every finite and pairwise disjoint family $\left(B_{i}\right)_{i \in I}$ of $\mathcal{B}\left(\mathbb{R}^{2}\right)$, the random variables $\left(\mathcal{N}\left(\cdot, B_{i}\right)\right)_{i \in I}$ are independent ;

(iii) for every bounded Borel set $B$ and every $k \in \mathbb{N}$

$$
\mathbf{P}_{\lambda}([\mathcal{N}(\cdot, B)=k])=\lambda^{k} \mathcal{L}_{2}(B)^{k} \frac{\exp \left(-\lambda \mathcal{L}_{2}(B)\right)}{k !} .
$$

We denote by $\mathbf{E}_{\lambda}$ the expectation operator with respect to the probability $\mathbf{P}_{\lambda}$. Note that for every bounded Borel set $B$ in $\mathbb{R}^{2}$, we have $\mathcal{N}(\omega, B)=\#(\Sigma \cap B)$, and that an easy calculation yields $\mathbf{E}_{\lambda}(\mathcal{N}(\cdot, B))=\lambda \mathcal{L}_{2}(B)$. For the existence of Poisson point processes and an explicit construction of the probability space $\left(\Sigma, \mathcal{A}, \mathbf{P}_{\lambda}\right)$, we refer the reader to 12 . We define the group $\left(T_{z}\right)_{z \in \mathbb{Z}^{2}}$ of $\mathbf{P}_{\lambda}$-preserving transformation on $\left(\Sigma, \mathcal{A}, \mathbf{P}_{\lambda}\right)$, by $T_{z} \omega=\omega-z$. From (ii) and using the mixing condition (41), we can easily show that $\left(\Sigma, \mathcal{A}, \mathbf{P}_{\lambda},\left(T_{z}\right)_{z \in \mathbb{Z}^{N}}\right)$ is ergodic. We claim, as we will see below, that the dynamical system $\left(\Sigma, \mathcal{A}, \mathbf{P}_{\lambda},\left(T_{z}\right)_{z \in \mathbb{Z}^{N}}\right)$ is a good description of the heterogeneous environment described above.

In a second step we define the random diffusion and the random reaction part. Given $R>0$, $\left(r^{-}, a^{-}, W^{-}\right)$and $\left(r^{+}, a^{+}, W^{+}\right)$in $[0,+\infty] \times[0, K] \times \operatorname{Conv}_{\alpha, \beta, \gamma}$, where $W^{-}, W^{+}$do not depend on $x$, we define the random density $W$ associated with the random diffusion part, by

$$
W(\omega, x, \xi)=W^{+}(\xi)+\left(W^{-}(\xi)-W^{+}(\xi)\right) \min \left(1, \mathcal{N}\left(\omega, B_{R}(x)\right)\right) .
$$

More explicitly we have

$$
W(\omega, x, \xi)=\left\{\begin{array}{l}
W^{-}(\xi) \text { if } x \in \underset{i \in \mathbb{N}}{\cup} B_{R}\left(\omega_{i}\right), \\
W^{+}(\xi) \text { otherwise. }
\end{array}\right.
$$

Similarly we define the random growth rate and the random threshold by

$$
\begin{aligned}
& r(\omega, x)=r^{+}+\left(r^{-}-r^{+}\right) \min \left(1, \mathcal{N}\left(\omega, B_{R}(x)\right)\right), \\
& a(\omega, x)=a^{+}+\left(a^{-}-a^{+}\right) \min \left(1, \mathcal{N}\left(\omega, B_{R}(x)\right)\right) .
\end{aligned}
$$

The random CP-structured reaction function is given by

$$
f(\omega, t, x, \zeta)=r(\omega, x) \frac{\zeta^{2}}{K}\left(1-\frac{\zeta}{K}\right)-r(\omega, x) a(\omega, x) \frac{\zeta}{K}\left(1-\frac{\zeta}{K}\right)
$$

which is a Fisher reaction function with Allee effect whose growth rate and threshold are $\left(r^{-}, a^{-}\right)$ when $x \in \cup_{i \in \mathbb{N}} B_{R}\left(\omega_{i}\right)$, and $\left(r^{+}, a^{+}\right)$otherwise. We set $W_{\varepsilon}(\omega, x, \xi)=W\left(\omega, \frac{x}{\varepsilon}, \xi\right)$, and $f_{\varepsilon}(\omega, t, x, \zeta)=$ $f\left(\omega, t, \frac{x}{\varepsilon}, \zeta\right)$. It is easy to check that conditions (42) and 43) hold so that the homogenized problem is still given by (54) where this time (when $N=2$ )

$$
\begin{aligned}
& \bar{r}=r^{-}+\left(r^{+}-r^{-}\right) \exp \left(-\lambda \pi R^{2}\right), \\
& \overline{r a}=r^{-} a^{-}+\left(r^{+} a^{+}-r^{-} a^{-}\right) \exp \left(-\lambda \pi R^{2}\right) .
\end{aligned}
$$

Endeed, using the fact that $\exists \omega \in \Sigma, y \in \bigcup_{i \in \mathbb{N}} B_{R}\left(\omega_{i}\right) \Longleftrightarrow \#\left(\Sigma \cap B_{R}(y)\right) \geq 1$, and by using Fubini's theorem, we have

$$
\begin{aligned}
\bar{r} & =\mathbf{E}_{\lambda}\left(\int_{Y} r(\omega, y) d y\right) \\
& =r^{+} \int_{\Sigma} \int_{(0,1)^{N}} \mathbb{1}_{\left[\#\left(\Sigma \cap B_{R}(y)\right)=0\right]}(\omega, y) d y d \mathbf{P}_{\lambda}(\omega)+r^{-} \int_{\Sigma} \int_{(0,1)^{N}} \mathbb{1}_{\left[\#\left(\Sigma \cap B_{R}(y)\right) \geq 1\right]}(\omega, y) d y d \mathbf{P}_{\lambda}(\omega) \\
& =r^{+} \int_{(0,1)^{N}} \int_{\Sigma} \mathbb{1}_{\left[\#\left(\Sigma \cap B_{R}(y)\right)=0\right]}(\omega, y) d \mathbf{P}_{\lambda}(\omega) d y+r^{-} \int_{(0,1)^{N}} \int_{\Sigma} \mathbb{1}_{\left[\#\left(\Sigma \cap B_{R}(y)\right) \geq 1\right]}(\omega, y) d \mathbf{P}_{\lambda}(\omega) d y \\
& =r^{+} \exp \left(-\lambda \pi R^{2}\right)+r^{-}\left(1-\exp \left(-\lambda \pi R^{2}\right)\right) .
\end{aligned}
$$

A similar calculation holds for $\overline{r a}$. 


\section{Appendix C. Proof of Lemma 4.1}

Since $\left(\psi_{n}\right)_{n \in \mathbb{N}}, \psi$ are uniformly proper, according to [8, Lemma 17.4.5], there exists $\mu>0$ such that $\psi_{n}+\mu\left(\|\cdot\|_{X}+1\right) \geq 0$ and $\psi+\mu\left(\|\cdot\|_{X}+1\right) \geq 0$ so that the integrals $\Psi_{n}$ and $\Psi$ are well defined for all $n \in \mathbb{N}$.

Furthermore, for sequences of convex proper and lower semicontinuous functions from a reflexive Banach $X$ spaces into $\mathbb{R} \cup\{+\infty\}$, where $X$ is as in the assumptions, there is equivalence between the Mosco-convergence and convergence of the sequences of the Moreau-Yosida approximations (see 7 , Theorem 3.26] or [16]). We are going to apply this result to the spaces $X$ and $L^{2}(0, T, X)$ which fulfill these conditions and to the functionals $\Psi_{n}, \Psi, \psi_{n}, \psi$ which are convex proper and lower semicontinuous.

Step 1. Denote by $\psi_{n}^{\lambda}, \psi^{\lambda}, \Psi_{n}^{\lambda}$, and $\Psi^{\lambda}$ the Moreau-Yosida approximation of index $\lambda>0$ of $\psi_{n}$, $\psi, \Psi_{n}$, and $\Psi$ respectively (for the definition and properties of Moreau-Yosida approximation see [8], Proposition 17.2.1]). For every $u \in L^{2}(0, T, X)$, we have

$$
\Psi_{n}^{\lambda}(u)=\int_{0}^{T} \psi_{n}^{\lambda}(u(t)) d t \quad \text { and } \quad \Psi^{\lambda}(u)=\int_{0}^{T} \psi^{\lambda}(u(t)) d t
$$

This result is an elementary case of interchange of infimum and integral (see for instance [6] and references therein).

Step 2. We claim that if $\psi_{n} \stackrel{M}{\rightarrow} \psi$ then $\Psi_{n} \stackrel{M}{\rightarrow} \Psi$. We have (see [7, Theorem 3.24])

$$
\psi_{n} \stackrel{M}{\rightarrow} \psi \Longleftrightarrow \forall u \in X \quad \forall \lambda>0 \quad \psi_{n}^{\lambda}(u) \rightarrow \psi^{\lambda}(u)
$$

Let $u \in L^{2}(0, T, X)$. Assume that $\psi_{n} \stackrel{M}{\rightarrow} \psi$. Then from above, for a.e. $t \in(0, T)$ and for all $\lambda>0$, we have $\psi_{n}^{\lambda}(u(t)) \rightarrow \psi^{\lambda}(u(t))$.

Let $v \in \operatorname{dom}(\psi)$. Since $\psi_{n} \stackrel{M}{\rightarrow} \psi$, there exists a sequence $\left(v_{n}\right)_{n \in \mathbb{N}}$ in $X$ such that $v_{n} \rightarrow v$ and $\psi_{n}\left(v_{n}\right) \rightarrow \psi(v)$. Then there exists $N \in \mathbb{N}$ which depends only on $\psi(v)$ and $\|v\|_{X}$ such that for all $n \geq N$

$$
\begin{aligned}
\psi_{n}^{\lambda}(u(t)) \leq \psi_{n}\left(v_{n}\right)+\frac{1}{2 \lambda}\left\|v_{n}-u(t)\right\|_{X}^{2} & \leq \psi(v)+1+\frac{1}{2 \lambda}\left\|v_{n}-u(t)\right\|_{X}^{2} \\
& \leq \psi(v)+1+\frac{1}{\lambda}\left\|v_{n}\right\|_{X}^{2}+\frac{1}{\lambda}\|u(t)\|_{X}^{2} \\
& \leq \psi(v)+2+\frac{1}{\lambda}\|v\|_{X}^{2}+\frac{1}{\lambda}\|u(t)\|_{X}^{2},
\end{aligned}
$$

where $\psi(v)+2+\frac{1}{\lambda}\|v\|_{X}^{2}+\frac{1}{\lambda}\|u(\cdot)\|_{X}^{2}$ belongs to $L^{1}(0, T)$. Then, according to the Lebesgue dominated convergence theorem, we deduce that for all $\lambda>0$

$$
\int_{0}^{T} \psi_{n}^{\lambda}(u(t)) d t \rightarrow \int_{0}^{T} \psi^{\lambda}(u(t)) d t
$$

that is, from Step 1, $\Psi_{n}^{\lambda}(u) \rightarrow \Psi^{\lambda}(u)$, which ends the proof since $u$ is arbitrary chosen in $L^{2}(0, T, X)$.

\section{REFERENCES}

[1] M. A. Ackoglu, U. Krengel. Ergodic theorem for superadditive processes. J. Reine Angew. Math. 323 (1981), 53-67.

[2] G. Allaire, A. Mikelic, A. Piatnitski. Homogenization approach to the dispersion theory for reactive transport through porous media. SIAM J. Math. Anal. 42:125-144, 2010.

[3] G. Allaire, I. Pankratova, A. Piatnitski. Homogenization of a nonstationary convection-diffusion equation in a thin rod and in a layer. SeMA Journal, 58(1):53-95, 2012.

[4] G. Allaire, I. Pankratova, A. Piatnitski. Homogenization and concentration for a diffusion equation with large convection in a bounded domain. J. Funct. Anal. 262: 300-330, 2012.

[5] Concentration phenomena for neutronic multigroup diffusion in random environments. Ann. Inst. H. Poincaré Anal. Non Linéaire, 30:419-439, 2013.

[6] O. Anza Hafsa and J.-P. Mandallena. Interchange of infimum and integral. Calc. Var. Partial Differential Equations, 18(4):433-449, 2003.

[7] H. Attouch. Variational convergence for functions and operators. Applicable Mathematics Series. Pitman (Advanced Publishing Program), Boston, MA, 1984.

[8] Hedy Attouch, Giuseppe Buttazzo, and Gérard Michaille. Variational analysis in Sobolev and BV spaces. MOS-SIAM Series on Optimization. Society for Industrial and Applied Mathematics (SIAM), Philadelphia, PA; Mathematical Optimization Society, Philadelphia, PA, second edition, 2014. Applications to PDEs and optimization. 
[9] Robert B. Banks. Growth and diffusion phenomena, volume 14 of Texts in Applied Mathematics. Springer-Verlag, Berlin, 1994. Mathematical frameworks and applications.

[10] H. Berestycki and P.-L. Lions. Some applications of the method of super and subsolutions. In Bifurcation and nonlinear eigenvalue problems (Proc., Session, Univ. Paris XIII, Villetaneuse, 1978), volume 782 of Lecture Notes in Math., pages 16-41. Springer, Berlin, 1980.

[11] H. Berestycki and P.-L. Lions. Une méthode locale pour l'existence de solutions positives de problèmes semi-linéaires elliptiques dans $\mathbf{R}^{N}$. J. Analyse Math., 38:144-187, 1980.

[12] N. Bouleau. Processus stochastiques et applications. Collection Méthodes. Hermann, 2000.

[13] H. Brézis. Opérateurs maximaux monotones et semi-groupes de contractions dans les espaces de Hilbert. NorthHolland Publishing Co., Amsterdam-London; American Elsevier Publishing Co., Inc., New York, 1973. North-Holland Mathematics Studies, No. 5. Notas de Matemática (50).

[14] E. Chabi and G. Michaille. Ergodic theory and application to nonconvex homogenization. Set-Valued Anal., 2(12):117-134, 1994. Set convergence in nonlinear analysis and optimization.

[15] D. Cioranescu, A. Piatnitski. Homogenization of Porus Medium with Randomly pulsating Microstructure. SeMA Journal, 58(1):53-95, 2012.

[16] Gianni Dal Maso. An introduction to $\Gamma$-convergence. Progress in Nonlinear Differential Equations and their Applications, 8. Birkhäuser Boston Inc., Boston, MA, 1993.

[17] G. Dal Maso, L. Modica. Nonlinear stochastic homogenization and ergodic theory. J. Reine Angew. Math. 363 (1986), $27-43$.

[18] U. Mosco. Convergence of convex sets and of solutions of variational inequalities. Advances in Math., 3:510-585, 1969.

[19] U. Mosco. On the continuity of the Young-Fenchel transform. J. Math. Anal. Appl., 35:518-535, 1971.

[20] Akira Okubo. Diffusion and ecological problems: mathematical models, volume 10 of Biomathematics. Springer-Verlag, Berlin-New York, 1980. An extended version of the Japanese edition, it Ecology and diffusion, Translated by G. N. Parker.

[21] C. V. Pao. Nonlinear parabolic and elliptic equations. Plenum Press, New York, 1992.

[22] B. Perthame, P.E. Souganidis A homogenization approach to flashing ratchets Nonlinear Differ. Equ. Appl. 18: 45-58, 2011.

[23] S. I. Pohožaev. The Dirichlet problem for the equation $\Delta u=u^{2}$. Soviet Math. Dokl., 1:1143-1146, 1960.

[24] S. Ruan. Delay differential equations in single species dynamics. In Delay differential equations and applications, volume 205 of NATO Sci. Ser. II Math. Phys. Chem., pages 477-517. Springer, Dordrecht, 2006.

[25] A. Tsoularis. Analysis of logistic growth models. volume 2 of Research Letters in the Information and Mathematical Sciences, pages 23-46. Massey University, 2001.

[26] P. Turchin. Quantitative Analysis of Movement: Measuring and Modeling Population Redistribution in Animals and Plants. Beresta Books, 2015. 